Aus der Abteilung Mund-, Kiefer- und Gesichtschirurgie

(Prof. Dr. med. Dr. med. dent. H. Schliephake)

im Zentrum Zahn-, Mund- und Kieferheilkunde

der Medizinischen Fakultät der Universität Göttingen

\title{
Klinische Resultate nach Sofortbelastung dentaler Implantate unter Anwendung kortikaler Mikrofixation
}

\author{
INAUGURAL-DISSERTATION
}

zur Erlangung des Doktorgrades

für Zahnheilkunde

der Medizinischen Fakultät

der Georg-August-Universität zu Göttingen

vorgelegt von

Felix Malinski

aus

Wilhelmshaven

Göttingen 2011 
Dekan: Prof. Dr. med. C. Frömmel

I. Berichterstatter: Prof. Dr. med. Dr. med. dent. W. Engelke II. Berichterstatter: Prof. Dr. med. dent. R. Mausberg III. Berichterstatter: Prof. Dr. med. Dr. rer. nat. T. Crozier Tag der mündlichen Prüfung: 25.05.2011 


\section{Inhaltsverzeichnis}

1 Einleitung 1

1.1 Stand der Wissenschaft . . . . . . . . . . . . . . 2

1.1.1 Versorgungsformen des zahnlosen Unterkiefers . . . . . . . . 2

1.1.2 Die Frühbelastung . . . . . . . . . . . . . 5

1.1.3 Die Sofortbelastung . . . . . . . . . . . . . . 10

1.1.4 Patientenzufriedenheit und Lebensqualität . . . . . . . . . 15

1.2 Das Konzept der Satellitenimplantate . . . . . . . . . . . . . 17

1.3 Zielsetzung der Arbeit . . . . . . . . . . . . . . . . . 19

2 Material und Methode $\quad 20$

2.1 Patientenkollektiv ....................... 20

2.2 Versorgung der Patienten . . . . . . . . . . . . . . . . . 21

2.2 .1 Implantatsysteme . . . . . . . . . . . . . . . 21

2.2 .2 Das Operationsverfahren . . . . . . . . . . . . . 22

2.3 Evaluationen . . . . . . . . . . . . . . . . 23

2.3.1 Verweildaueranalyse und Erfolgskriterien . . . . . . . . . . 23

2.3.2 Beurteilung der periimplantären Gingiva . . . . . . . . . . . 24

2.3.3 Das Periotestgerät . . . . . . . . . . . . . . 24

2.3.4 Bestimmung der Periotestwerte ............. 25

2.3.5 Bestimmung des periimplantären Knochenabbaus . . . . . . . 25

2.3.6 Patientenfragebögen ................... 28

3 Resultate 31

3.1 Das Patientenkollektiv der Nachuntersuchung . . . . . . . . . . . . 31

3.2 Lebensdaueranalyse . . . . . . . . . . . . . . . . . . . . 32

3.3 Ergebnisse des Periotests . . . . . . . . . . . . . . . 33

3.4 Ergebnisse des horizontalen Knochenabbaus . . . . . . . . . . . 37

3.5 Ergebnisse der Sondierungstiefen . . . . . . . . . . . . . . 40

3.6 Blutung auf Sondierung . . . . . . . . . . . . . . 43

3.7 Fragebogen zur Patientenzufriedenheit . . . . . . . . . . . . . 44

3.7.1 Gesamtresultat der Behandlung . . . . . . . . . . . . . . 44

3.7.2 Auswertung des Halts der Prothese mit Kugelattachments . . . 45 
3.7.3 Bewertung der Kaufunktion . . . . . . . . . . . . . 46

3.7.4 Bewertung der Reinigung der Prothese und Implantate . . . . . 47

3.7.5 Anzahl der Zahnarztbesuche aufgrund von Komplikationen . . . 48

3.7.6 Nachfrage zur Behandlungsempfehlung des Göttinger Konzepts 49

3.7.7 Nachfrage zur Zufriedenheit mit der Mundgesundheit . . . . . 49

3.8 Auswertung des OHIP-Fragebogens . . . . . . . . . . . . . 49

3.9 Falldokumentation einer Patientin . . . . . . . . . . . . . . 51

4 Diskussion $\quad 55$

4.1 Beurteilung der Überlebenszeitanalyse und Erfolgsquote . . . . . . . . . 55

4.2 Beurteilung der Implantatstabilität . . . . . . . . . . . . . . . 57

4.3 Beurteilung des Knochenabbaus . . . . . . . . . . . . 58

4.4 Beurteilung der Sondierungstiefen . . . . . . . . . . . . . . 61

4.5 Beurteilung der Patientenzufriedenheit und mundgesundheitsbezogenen Lebensqualität . . . . . . . . . . . . . . . . 62

4.6 Schlussfolgerung . . . . . . . . . . . . . . . 65

5 Zusammenfassung $\quad 66$

6 Anhang $\quad 68$

6.1 Abkürzungsverzeichnis . . . . . . . . . . . . . . 68

6.2 Tabellenverzeichnis . . . . . . . . . . . . . . . . . . . . 69

6.3 Abbildungsverzeichnis . . . . . . . . . . . . . . . 69

$\begin{array}{lll}7 & \text { Literaturverzeichnis } & 71\end{array}$ 


\section{Einleitung}

Die prothetische Versorgung des zahnlosen atrophierten Unterkiefers erweist sich auch heute noch als problematisch und ist in vielen Fällen, sowohl für den Behandler als auch den Patienten, unbefriedigend. Mit der Entwicklung dentaler Titanimplantate wurden jedoch die Versorgungsmöglichkeiten zahnloser Patienten durch implantat-retinierten herausnehmbaren oder festsitzenden Zahnersatz deutlich erweitert und verbessert. Dabei konnten besonders die herausnehmbaren implantatgestüzten Deckprothesen im Unterkiefer die Zufriedenheit und das Kauvermögen der Patienten signifikant steigern (Awad und Feine (1998), Assunção et al. (2009), Heydecke et al. (2003)).

Das von Brånemark (1983) aufgestellte klassische Belastungsprotokoll empfiehlt ein zwei-phasiges operatives Vorgehen und eine Einheilungszeit von mindestens drei bis sechs Monaten, bevor eine Belastung der Implantate durch eine Prothese möglich ist (Brånemark (1983)). Dennoch wird heutzutage die von Branemark geforderte Einheilungszeit, um eine erfolgreiche Osseointegration zu ermöglichen, kontrovers diskutiert. Diese Entwicklung ist vor allem auf die Wünsche und Forderungen der Patienten nach einer raschen Behandlung mit optimalen funktionellen und ästhetischen Ergebnissen bei gleichzeitiger Kostenreduzierung zurückzuführen (Henry et al. (2003)). Die Probleme, die mit einer längeren Einheilungszeit einhergehen, führen bei vielen Patienten zu einer Ablehnung einer implantologischen Versorgung (Raghoebar et al. (2003)). So darf bei dem Konzept der konventionellen submukösen Einheilung dentaler Implantate während der ersten zwei Wochen nach Implantation keine Prothese getragen werden. Dies führt bei den Patienten zu einer deutlichen Einschränkung der Lebensqualität in Hinblick auf die Kaufunktion, Phonetik und Ästhetik und stellt für viele Patienten eine starke psychische Beeinträchtigung dar (Rungcharassaeng und Kan (2000)).

Vor diesem Hintergrund beschäftigten sich in der Vergangenheit zahlreiche Studien mit alternativen Behandlungsprotokollen und strebten eine sofortige Belastung von Implantaten an. Ledermann (1979) und später auch Brånemark et al. (1999) zeigten, dass die primäre Verblockung von 3-4 Implantaten durch eine Stegkonstruktion, zu einer Reduktion der Mikrobewegungen und nicht-axialen Belastungskräften am Implantat führt und so eine erfolgreiche Osseointegration ermöglicht. Zahlreiche aktuelle Studien bestätigten diese Ergebnisse und berichteten von durchschnittlichen Erfolgsraten von über 95 \% (Ormianer et al. (2006), Degidi et al. (2007), Stoker und Wismeijer 
(2009)).

Engelke et al. (2005) zeigten, dass eine primäre Verblockung von Implantaten nicht nur durch supragingivale Stegkonstruktionen möglich ist. Durch den Verbund kleiner kortikaler Osteosyntheseschrauben mit dem Implantat gelang es ebenfalls eine ausreichende Primärstabilität zu erreichen und eine Sofortbelastung zu ermöglichen. Die ersten Untersuchungsergebnisse dieser prospektiven Studie zeigten nach einem Beobachtungszeitraum von 10 Monaten eine Implantatüberlebensrate von $100 \%$. Demzufolge ist das Konzept der subgingivalen Verblockung durch sogenannte Satellitenimplantate mit dem von Ledermann (1979) und Brånemark et al. (1999) vergleichbar.

Im Rahmen dieser prospektiven Studie soll eine weitere Nachuntersuchung des Patientenkollektivs nach einem mittleren Untersuchungszeitraum von 52 Monaten Aufschluss über den längerfristigen Erfolg dieses Konzepts geben. Besonderes Augenmerk soll dabei auf die Zufriedenheit und orale Lebensqualität der Patienten gerichtet werden.

\subsection{Stand der Wissenschaft}

\subsubsection{Versorgungsformen des zahnlosen Unterkiefers}

Der zahnlose Unterkiefer gilt schon sehr lange als klassisches Indikationsgebiet für implantatretinierten Zahnersatz. Zahlreiche Studien bestätigten dies und zeigten in der Vergangenheit zuverlässige und erfolgreiche Ergebnisse. Dabei gilt besonders die stark atrophierte zahnlose Mandibula mit insuffizientem Prothesenlager als absolute Indikation für eine Implantation (Tetsch et al. (1990)).

Dies liegt vor allem daran, dass die prothetische Versorgung zahnloser atrophierter Unterkiefer sich auch heute noch als sehr problematisch darstellt und in vielen Fällen, sowohl für den Behandler als auch den Patienten unbefriedigend ist. Darüber hinaus erreichten die in den 80er-Jahren relativ aufwendig durchgeführten präprothetischchirurgischen Maßnahmen zur Verbesserung des Prothesenlagers langfristig gesehen keine Verbesserung und neigten gerade im Unterkiefer zu einer hohen Rezidivrate. So wurde bei einer Studie von Koberg (1985) bei einer Nachuntersuchung von Patienten mit einer Unterkieferalveolarkammplastik mittels Rippentransplantat nach fünf Jahren eine vollständige Resorption des augmentierten Knochennivaus festgestellt. Daher werden heutzutage diese präprothetisch-chirurgischen Maßnahmen nicht mehr zur Verbesserung des Prothesenlagers, sondern nur bei entsprechender Indikation in Kombination mit einer implantatgestützten Versorgung durchgeführt.

Es werden grundsätzlich zwei Versorgungskonstruktionen unterschieden. Zum einen die implantatgestütze, jedoch hauptsächlich gingival gelagerte Deckprothese und zum 
anderen die implantatgetragene bedingtabnehmbare Brückenkonstruktion (Reinhardt und Drüke (1993)). Dabei hat sich vor allem die herausnehmbare implantatgestützte Deckprothese aufgrund der konzeptionellen Vielseitigkeit, einfachen Durchführung für den Behandler und sehr erfolgreichen Ergebnissen, zu einer Art Standardversorgung für stark atrophierte Unterkiefer etabliert. Abgesehen von der festsitzenden reinimplantatgetragenen Brückenversorgung lassen sich drei verschiedene Konzepte unterscheiden.

Das erste Konzept stellt die Minimalversorgung dar und ist bei mäßiger Kieferkammatrophie sowie bei Patienten mit Retentionsproblemen ihrer Prothese indiziert. Es werden zwei Implantate in der Regio interforaminalis inseriert. Die Prothese wird anschließend über zwei Kugelattachments auf den Implantaten und den entsprechenden Matrizen in der Prothese befestigt (Vercruyssen et al. (2010), Cooper et al. (2008), Jennings (1991)). Die Vorteile dieser Art der Versorgung sind der geringe Platzbedarf, die einfache Konstruktion, relativ geringe Kosten und die gute Hygienefähigkeit für den Patienten. Eine erhebliche Kostenreduktion entsteht vor allem durch die Möglichkeit der Umarbeitung der bereits vorhandenen Prothese des Patienten (Engelke et al. $(2005))$.

Eine weitere Möglichkeit zur Befestigung der Prothese, basierend auf dem Konzept zweier interforaminaler Implantate, besteht in der Verwendung einer geradlinigen Stegverbindung. Dabei sollte diese eine Länge von mindestens $20 \mathrm{~mm}$ haben und den Zungenraum nicht einschränken. Die Verbindung zur Deckprothese wird über einen in die Prothese eingearbeiteten aktivierbaren Stegreiter ermöglicht (Block et al. (1990)). Vorteile dieser Ausführung sind ebenfalls die einfache Konstruktion des konfektionierten Stegs, sowie die Verblockung der Implantate. Payne et al. (2002) und Tawse-Smith et al. (2002) zeigten, dass durch die Verblockung der Implantate auch eine Frühbelastung möglich ist. Als Nachteil dieses Retentionselements muss allerdings die schlechtere Hygienefähigkeit für den Patienten genannt werden.

Da bei manchen Patienten mit geringerem Knochenangebot und schmaler Unterkieferspange nicht immer der geforderte Mindestabstand von $20 \mathrm{~mm}$ realisiert werden kann, besteht weiterhin die Möglichkeit an den zwei Implantaten jeweils eine Stegextension zu befestigen. Dadurch wird trotz des kürzeren Stegs die Rotationstendenz der Deckprothese verringert. Mericske-Stern (1997) zeigte jedoch, dass nur durch die Verwendung von Abstandshaltern zwischen den Stegextensionen und den Matrizen in der Prothesenbasis das Frakturrisiko der Extensionen minimiert werden kann. Sowohl bei der Verwendung von Kugelattachments als auch einer Stegverbindung ohne Extension wird die Deckprothese frontal-linear abgestützt. Bei Kaubelastungen kommt es zu einem Einsinken der Prothese nach posterior. Sie ist daher überwiegend gingival gelagert, sodass es zu keiner kritischen Belastung der Implantate kommt (Heckmann et al. (2001)). 
Das zweite Konzept stellt die Standardversorgung für implantatretinierten Zahnersatz im Unterkiefer dar und ist sowohl bei geringer bis hin zur hochgradigen Kieferkammatrophie indiziert. Dabei werden vier Implantate in der Regio interforaminalis mit gleich großen Abständen von ca. 12 mm inseriert. Diese können dann sowohl mit Kugelkopfattachments als auch mit einer Stegkonstruktion versorgt werden. Bei der Stegverbindung entsteht durch die versetzte Anordnung der vier Implantate ein Winkel, wodurch nicht nur eine horizontale, sondern auch eine sagittale Abstützung ermöglicht wird. Diese Art der Versorgung ist wesentlich stabiler als eine Stegkonstruktion auf zwei interforaminalen Implantaten und ermöglicht auch eine Sofortbelastung. So konnte in zahlreichen Studien eine erfolgreiche Osseointegration der Implantate nachgewiesen werden (Ledermann (1977), Brånemark et al. (1999), Degidi et al. (2007)). Als Nachteil dieser Konstruktion sind die erhöhten Kosten für den Patienten und die schlechtere Hygienefähigkeit zu nennen. Bei der Verwendung von vier Kugelattachments entstehen geringere Kosten für den Patienten und ihm wird eine bessere Hygienefähigkeit ermöglicht. Auf Grund der fehlenden Verblockung der Implantate ist allerdings eine Sofortbelastung nicht möglich.

Das dritte Behandlungskonzept beschreibt die Insertion von vier bis fünf Implantaten, welche über einen Steg mit distalen Extensionen verblockt werden. Es ist vor allem bei einer gravierenden horizontalen Kieferkammatrophie indiziert. Auf Grund der relativ weiten Extension nach distal ist die Deckprothese nahezu vollständig implantatgetragen.

Die wesentlichen Vorteile dieser Konstruktion sind die sehr hohe Stabilität und der feste Halt der hauptsächlich implantatgetragenem Deckprothese, welcher annähernd mit dem einer festsitzenden implantatretinierten Brückenkonstruktion zu vergleichen ist. Zusätzlich können durch die Verwendung einer Deckprothese auch schwierige anatomische Ausgangssituationen bei Patienten ausgeglichen werden. 


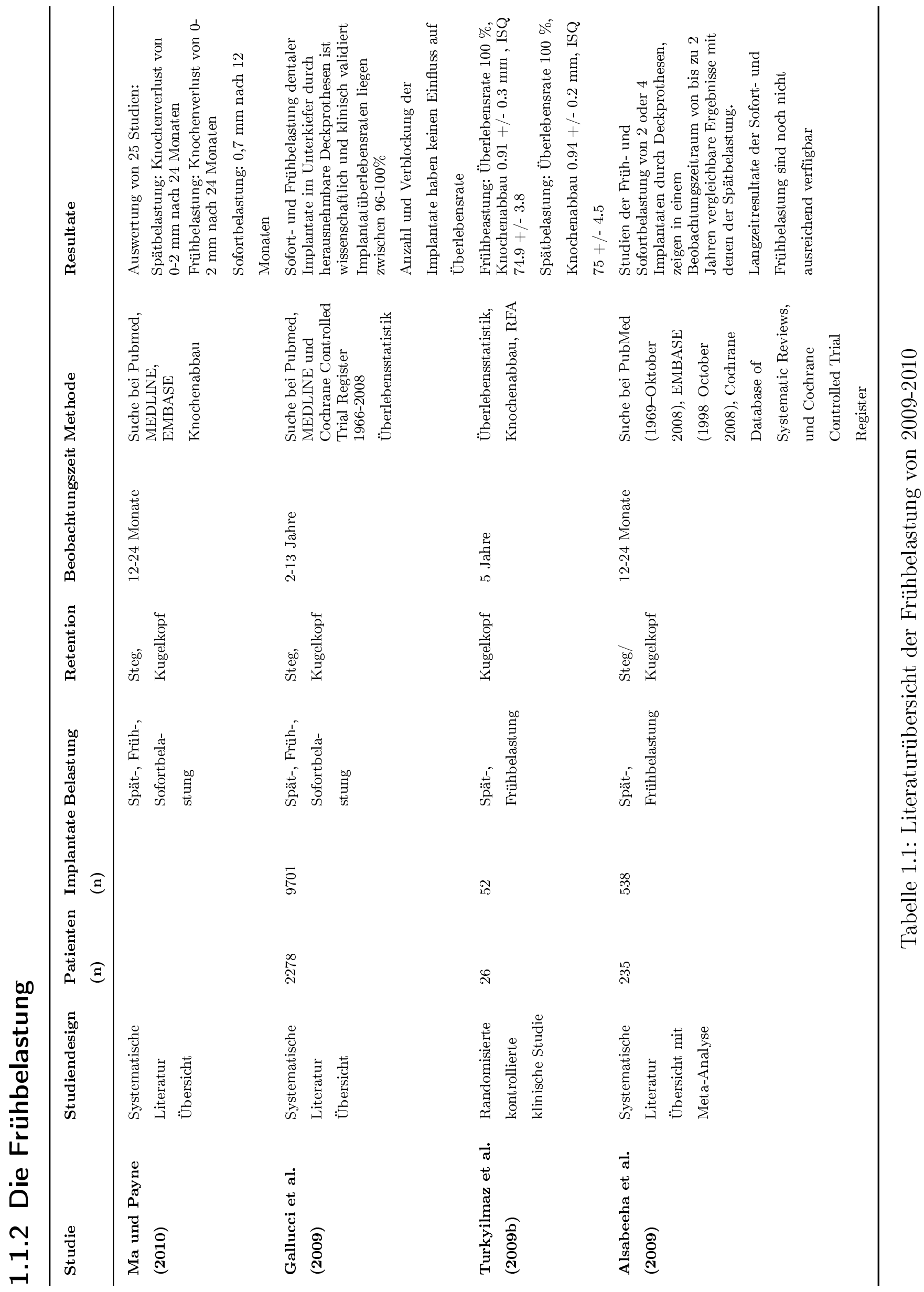




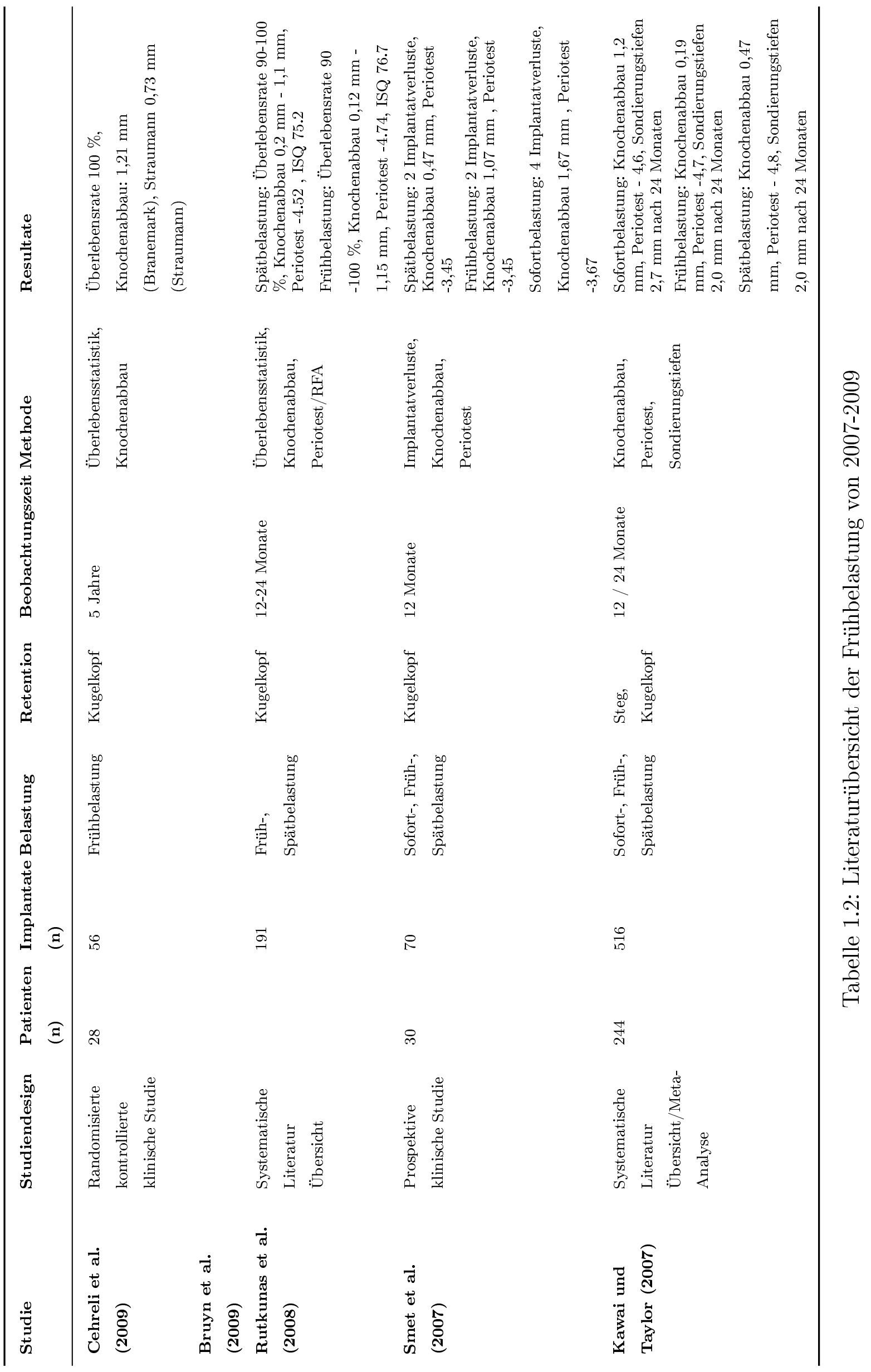


Die Frühbelastung ist im Vergleich zur Spät- und Sofortbelastung nicht eindeutig in der Literatur definiert. Auf der 3. ITI-Konsensuskonferenz in Gstaad, Schweiz (ITIKonsensuskonferenz 2004) wurde die Frühbelastung als Belastung eines Implantats innerhalb des Zeitraums von 48 Stunden bis drei Monate nach Insertion definiert. Verschiedene Autoren legen den Zeitpunkt der Belastung innerhalb dieses Zeitraums unterschiedlich fest.

So definierten Cehreli et al. (2009) und Turkyilmaz et al. (2009b) den Zeitpunkt der Belastung nach sieben Tagen, Friberg et al. (2005) nach 13 Tagen, Engelke et al. (2002) nach 21 Tagen und Tawse-Smith et al. (2002) erst nach sechs Wochen.

Payne et al. (2002) publizierten eine Arbeit über Frühbelastung von interforaminalen Implantaten durch eine Deckprothese. In diesem Zusammenhang wurden bei 10 Patienten jeweils zwei Brånemark-Implantate in der Regio interforaminalis inseriert. Nach 14 Tagen wurden diese über Kugelattachments durch eine Deckprothese belastet. Nach 52 Wochen waren noch alle inserierten Implantate in situ. Der mittlere Knochenabbau betrug an den Implantaten mesial 0,86 $\mathrm{mm}$ und distal 1,13 $\mathrm{mm}$.

Friberg et al. (2005) veröffentlichten die Ergebnisse einer retrospektiven Vergleichsstudie. In dieser wurden bei 152 Patienten insgesamt 750 Implantate gesetzt, die nach 13 Tagen durch eine Deckprothese belastet wurden. Das Patientenkollektiv wurde zuvor in eine Kontroll- und Testgruppe unterteilt. Die Kontrollgruppe wurde nach dem klassischen zwei-zeitigen Operationsverfahren behandelt. Nach einem Jahr wurde in der Testgruppe eine Erfolgsrate von 97,5 \% und in der Kontrollgruppe von 99,7 \% festgestellt. Der mittlere Knochenverlust wurde nach einem Jahr mit 0,4 mm für beide Gruppen angegeben.

Da sich nur sehr wenige Studien in der Literatur mit unverblockten frühbelasteten Implantaten befasst haben, verglichen Turkyilmaz et al. (2006a) die Frühbelastung mit dem klassischen Belastungsprotokoll. Dabei erhielten 26 Patienten jeweils zwei Implantate in der interforaminalen Region. Dreizehn Patienten wurden anschließend nach dem klassischen Konzept mit einer Implantateinheilungszeit von drei Monaten behandelt. Die zweite Patientengruppe wurde direkt nach der Implantation mit Kugelattachments versorgt. Nach fünf Tagen wurden die Implantate mit einer Deckprothese belastet. Nach 24 Monaten konnten in der Test- und Kontrollgruppe keine signifikanten Unterschiede bezüglich des horizontalen Knochenabbaus festgestellt werden. Die Überlebensrate betrug in beiden Gruppen $100 \%$.

Drei Jahre später veröffentlichten Turkyilmaz et al. (2009b) weitere Ergebnisse dieser Studie nach einem Beobachtungzeitraum von fünf Jahren. In beiden Gruppen wurde ein horizontaler Knochenabbau von $0,93 \mathrm{~mm}+/-$ 0,3 mm festgestellt. Auch nach fünf Jahren betrug die Implantatüberlebensrate in beiden Gruppen 100 \%. Die Behandlungsrgebnisse der Frühbelastung unverblockter Implantate wurden mit denen der 
Spätbelastung gleichgesetzt.

Arvidson et al. (2008) inserierten bei 62 Patienten 250 Implantate in der Regio interforaminalis des Unterkiefers. Dabei erhielten 60 Patienten jeweils vier Implantate und zwei Patienten fünf Implantate. Das Durchschnittsalter des Patientenkollektivs betrug 68 Jahre. Die Implantate wurden durchschnittlich nach 6,6 Tagen mit einer festsitzenden verblockten Brückenkonstruktion versehen. Nach drei Jahren betrug die Überlebensrate der Implantate 98,55 \% und der Knochenverlust 0,73 mm. Als wichtigstes Kriterium für die hohe Erfolgsrate dieser Studie wurde eine hohe Primärstabilität und feste Verblockung der Implantate während der Einheilungszeit genannt.

In einer Studie von Cehreli et al. (2009) wurden bei 28 Patienten (6 Männer und 22 Frauen, Durchschnittsalter 64 Jahre) jeweils zwei interforaminale Implantate gesetzt, die unverblockt nach einer Woche belastet wurden. Das Patientenkollektiv wurde in zwei Gruppen unterteilt, um außerdem zwei Implantatsysteme (Brånemark, Straumann) vergleichen zu können. Nach einem Untersuchungszeitraum von fünf Jahren lag die Überlebenswahrscheinlichkeit für beide Implantatsysteme bei 100 \%. Der Knochenverlust betrug beim Brånemark-Implantatsystem durchschnittlich 1,21 mm und beim Straumann- Implantatsystem 0,73 mm.

Bruyn et al. (2009) inserierten bei 37 Patienten jeweils zwei Implantate in der interforaminalen Region. Nach 7-10 Tagen wurden die Implantate mit Kugelattachments und einer Deckprothese versorgt und belastet. Nach einer Beobachtungszeit von 18 Monaten wurde ein Implantatverlust festgestellt. Die Überlebensrate betrug $97 \%$. Es wurde im Mittel ein horizontaler Knochenverlust von 0,8 mm +/- 0,48 mm gemessen. $90 \%$ der Patienten waren zufrieden mit dem Gesamtergebnis ihrer Behandlung. Insgesamt wurde das Behandlungskonzept als praktikabel und erfolgversprechend eingeschätzt.

In einer Arbeit von Turkyilmaz et al. (2009a) wurden bei sieben Patienten (Durchschnittsalter 58 Jahre) jeweils fünf interforaminale Implantate gesetzt. Nach vier Tagen wurden diese über einen Steg verblockt und mit einer Hybridprothese belastet. Um eine Abdrucknahme kurz nach der Implantatinsertion zu vermeiden, wurde ein eigens entwickelter Kunststoffrahmen verwendet (Ohio-State-University-acrylic-frame). So sollte ein möglicher Schaden der periimplantären Gingiva durch ein Abdruckmaterial verhindert werden. Ihrer Ansicht nach sei dieses Konzept durch seine Variabilität auch bei schwierigen anatomischen Verhältnissen anwendbar. Nach 19 Monaten betrug die Überlebensrate der Implantate $100 \%$ und der mittlere Knochenverlust weniger als $1 \mathrm{~mm}$.

Alsabeeha et al. (2009) untersuchten in einer Literaturübersicht mit Meta-Analyse Studien, die im Zeitraum von 1969 bis 2009 die Früh- und Sofortbelastung implantatgestützer Deckprothesen des Unterkiefers mit dem konventionellen Belastungsprotokoll verglichen. Die Literaturrecherche wurde über PubMed, EMBASE, Cochrane Data- 
base of Systemic Reviews und im Cochrane Controlled Trial Register durchgeführt. Von 191 Studien erfüllten nur zehn Studien die Auswahlkriterien für die Analyse, wobei sieben Studien die Frühbelastung und drei Studien die Sofortbelastung mit dem klassischen Belastungskonzept verglichen. Die Meta-Analyse zeigte bei den sieben Studien der Frühbelastung keine statistisch signifikant höheren Implantatverlustraten, als beim konventionellen Belastungsprotokoll. Auch bei den Studien der Sofortbelastung konnten keine signifikanten Unterschiede der Implantatverlustrate zum klassischen Verfahren festgestellt werden. Zusammenfassend zeigte die Literaturübersicht und MetaAnalyse, dass die Belastungsprotokolle der Früh- und Sofortbelastung für implantatgestütze Deckprothesen im Unterkiefer mit vergleichbarem Erfolg zum konventionellen Verfahren durchführbar sind. 


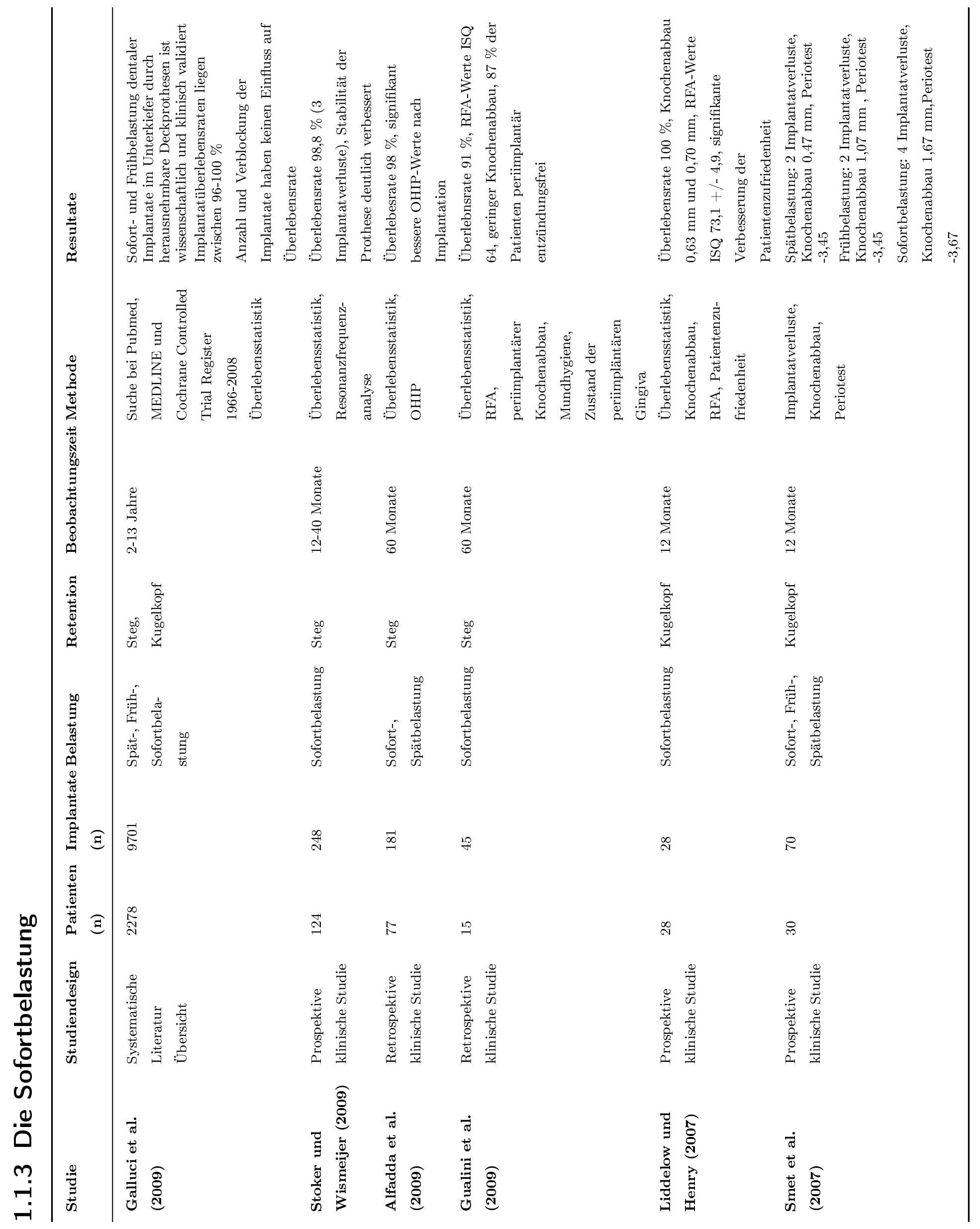

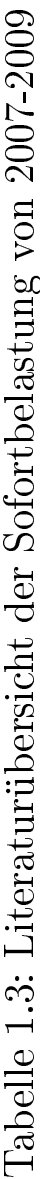




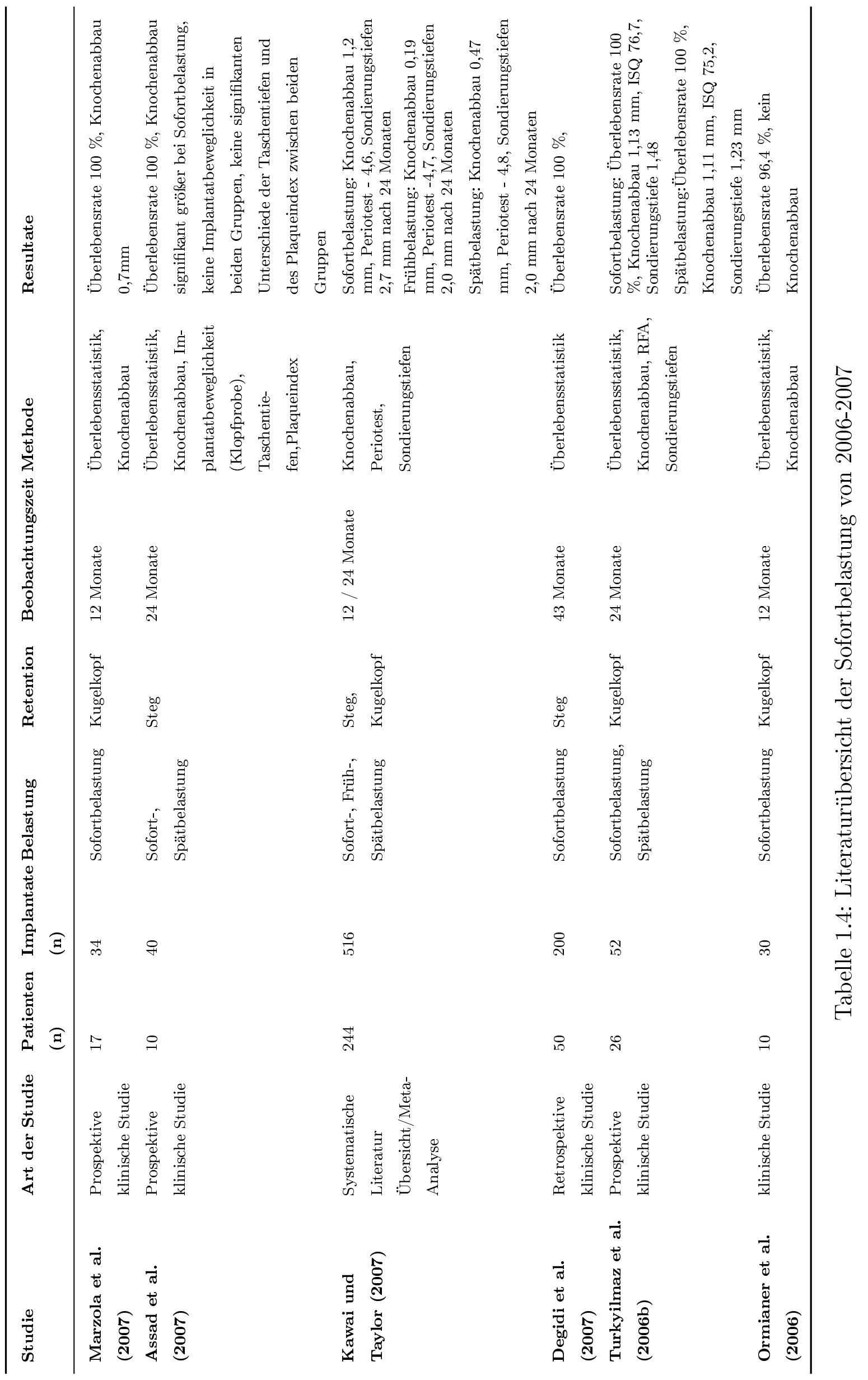


Engelke et al. (2002) definierten die Sofortbelastung als die funktionelle Belastung eines Implantats innerhalb eines Zeitraums von 48 Stunden nach Implantatinsertion durch einen provisorischen oder definitiven Zahnersatz mit Okklusionskontakt.

Auf der 3. ITI-Konsensuskonferenz in Gstaad, Schweiz (ITI-Konsensuskonferenz 2004) wurde die Sofortbelastung ebenfalls als Eingliederung eines Zahnersatzes unter Okklusionskontakt bis spätestens 48 Stunden nach Implantation beschrieben.

Eine weitere Definition des Terminus Sofortbelastung wurde auf der 1. Europäischen Konsensuskonferenz Implantologie 2006 in Köln (BDIZ 2006) vorgenommen, welche auf den Ergebnissen des Implants World Congress Consensus Meeting in Barcelona 2002, der 3. ITI-Konsensuskonferenz in Gstaad 2003 und der Immediate Function Consensus Conference in Hollywood 2003 sowie eigener Studien der Teilnehmer basierte. Dabei wurde die Sofortbelastung als die Eingliederung von Zahnersatz mit Okklusionskontakt am Tag der Implantation oder bis spätestens 72 Stunden nach der Implantation definiert.

Die Sofortbelastung dentaler Implantate ist in der Literatur gut dokumentiert und wird heutzutage hinsichtlich der Überlebensdauer mit den konventionellen Belastungsprotokollen gleichgesetzt. Besonders die Sofortbelastung dentaler Implantate in der Regio interforaminalis der Mandibula mit einer Deckprothese hat in zahlreichen Studien vielversprechende Ergebnisse gezeigt.

So beschrieb schon Ledermann (1977) in diesem Zusammenhang die Sofortbelastung von verblockten titanplasmabeschichteten Schraubenimplantaten im interforaminalen Bereich des Unterkiefers. 1996 publizierte er weitere Ergebnisse verschiedener Implantattypen, die am Tag der Insertion mit einem Steg verblockt und anschließend mit einer Deckprothese belastet wurden. Die Überlebensrate dieser Studie betrug $90 \%$.

Brånemark et al. (1999) stellten mit dem Novum Protokoll ein Therapiekonzept vor, das eine vollständige Wiederherstellung der Kaufunktion des zahnlosen Unterkiefers innerhalb eines Tages ermöglichte. In dieser Studie wurden bei 90 Patienten 270 BranemarkNovum-Implantate in der interforaminalen Region gesetzt, die noch am gleichen Tag mit einer konfektionierten Brücke versorgt wurden. Nach einem Beobachtungszeitraum von drei Jahren betrug die Erfolgsrate 98 \%. Auch der Knochenabbau betrug nach einem Jahr im Mittel weniger als $1 \mathrm{~mm}$. Diese Ergebnisse waren vergleichbar mit denen des klassischen zweiphasigen Belastungskonzepts.

In einer Arbeit von Ormianer et al. (2006) wurde ein modifiziertes Sofortbelastungskonzept erprobt. Dabei wurde versucht, die auf die Implantate einwirkenden Kaukräfte so zu minimieren, dass auch ohne Verblockung der Implantate eine Sofortbelastung möglich ist. Dafür wurden bei 10 Patienten jeweils 3 Implantate im interforaminalen Bereich gesetzt, wobei nur zwei Implantate sofort belastet wurden und ein Implantat 
als „Ersatzimplantat“ unbelastet einheilen sollte. Anschließend wurde die Prothese über Kugelanker mit den distalen Implantaten verbunden. Um eine Reduktion der Kaukräfte auf die Implantate zu erreichen, wurde anstelle der Matrizen das Abdruckmaterial Impregum (3M Espe, Seefeld, Deutschland) für die ersten drei Monate als Retentionsund Dämpfungselement benutzt. Nach 12 Monaten kam es zu einem Implantatverlust, so dass sich eine Erfolgsrate von 96,4 \% ergab. Es wurde berichtet, dass durch die Verwendung des Abdruckmaterials die Kaukräfte auf die Implantate reduziert wurden und dadurch die Wahrscheinlichkeit einer erfolgreichen Osseointegration der Implantate erhöht wurde.

Degidi et al. (2007) inserierten bei 50 Patienten (28 Frauen u. 22 Männer im Alter von 42-77 Jahren) im Unterkiefer jeweils vier interforaminale Implantate, welche durch eine Stegkonstruktion miteinander verblockt und anschließend mit einer Deckprothese sofort belastet wurden. Nach einem Beobachtungszeitraum von 43 Monaten wurde ein durchschnittlicher Knochenabbau von $0.8 \mathrm{~mm}$ festgestellt. Die Implantatüberlebensrate in der Studie lag bei 100 \%. Grundsätzlich wurde das Konzept der Verblockung von vier interforaminalen Implantaten und der sofortigen Belastung als gut reproduzierbar und zuverlässig bewertet. Des Weiteren wurde festgestellt, dass längere und breitere Implantate einen positiven Einfluss auf die Knochenresorption am Implantat hätten.

In einer Studie von Marzola et al. (2007) erhielten 17 Patienten (11 Frauen, 6 Männer, Durchschnittsalter: 65,82 Jahre) jeweils zwei Implantate (MK III TiUnite) in der Regio interforaminalis, die darauf mit Kugelattachments und einer Deckprothese versorgt und sofort belastet wurden. Im Gegensatz zu den meisten Studien wurde keine primäre Verblockung der Implantate durch einen Steg oder zusätzliche Satellitenimplantate vorgenommen. Nach einem mittleren Beobachtungszeitraum von 12 Monaten wurde ein Knochenabbau von durchschnittlich 0,7 mm gemessen. Die Kaplan-Meier Überlebensstatistik betrug $100 \%$. Insgesamt wurde das Ergebnis dieser Studie innerhalb des beschriebenen Beobachtungszeitraums als erfolgreiche und zuverlässige Alternative zur konventionellen zweiphasigen Behandlung beschrieben. Wichtigste Voraussetzungen für das positive Behandlungsergebnis seien eine ausreichende Primärstabilität und gute Verteilung der Kaukräfte durch eine sorgfältige funktionelle Anpassung der Prothese.

Smet et al. (2007) verglichen anhand von drei Patientengruppen mit jeweils zehn Patienten die Konzepte der Spät-, Früh- und Sofortbelastung. Die Patientengruppen der Spätbelastung und Frühbelastung erhielten jeweils zwei interforaminale Implantate. Als Verankerungselement für die Deckprothese wurden Kugelattachments verwendet. Die Patientengruppe der Sofortbelastung wurde nach dem Novum Konzept von Brånemark et al. (1999) mit jeweils drei Implantaten versorgt. Die Patienten der drei Gruppen durften dann, entsprechend ihres Belastungsprotokolls, die Implantate nach 
vier Monaten, einer Woche oder einem Tag nach Implantation mit einer Deckprothese belasten. Nach einer Beobachtungszeit von einem Jahr, kam es in der Gruppe der Spätund Frühbelastung zu jeweils zwei Implantatverlusten. In der Gruppe der Sofortbelastung gingen vier Implantate verloren. Trotz der höheren Implantatverlustrate in der Gruppe der Sofortbelastung, konnte kein statistisch signifikanter Unterschied zwischen der Verlustrate und den verschiedenen Belastungsprotokollen festgestellt werden.

In einer Literaturübersicht mit Meta-Analyse von Kawai und Taylor (2007) wurde die Früh- und Sofortbelastung mit dem konventionellen Belastungsprotokoll verglichen. Dazu wurden nur Studien ausgewertet, die sich auf implantatgestütze Deckprothesen des Unterkiefers bezogen. Insgesamt konnten von 239 nur neun Studien ausgewählt werden, die alle festgelegten Auswahlkriterien erfüllten. Aus ihnen wurden die Daten des horizontalen Knochenabbaus, der Sondierungstiefen, der Plaqueindizes, sowie Blutungsindizes ausgewertet und miteinander verglichen. Bei der Analyse konnten keine statistisch signifikanten Unterschiede der klinischen Parameter im Zusammenhang mit einer Sofortbelastung und konventionelle Belastung festgestellt werden. Zusammenfassend wurde das konventionelle Belastungsprotokoll mit einer Einheilungszeit von drei Monaten als das am besten dokumentierte, allerdings nicht einzige annehmbare Konzept dargestellt. So zeigen auch die Sofort- und Frühbelastung nach einer Beobachtungszeit von 24 Monaten keine erkennbaren negativen Einflüsse auf das Behandlungsergebnis.

Stoker und Wismeijer (2009) behandelten 124 Patienten nach einem neuen Behandlungskonzept, das ebenfalls eine Sofortbelastung interforaminal gesetzter Implantate beabsichtigte. Dazu wurden allen Patienten jeweils zwei interforaminale Implantate inseriert, die noch am gleichen Tag mit einer Stegkonstruktion und Deckprothese versorgt wurden. Nach einem Beobachtungszeitraum von 12-40 Monaten gingen drei der 248 Implantate verloren. Die Überlebensrate der Implantate betrug 98,8 \%. Insgesamt wurde die Belastung zweier verblockter interforaminaler Implantate durch eine Deckprothese als erfolgversprechend und zuverlässig bewertet.

In einer Arbeit von Alfadda et al. (2009) wurde die Sofortbelastung mit dem klassischen Belastungskonzept verglichen. In der Testgruppe befanden sich 35 Patienten, die mit jeweils zwei Implantaten in Regio 033, 043 und einer konfektionierten Stegkonstruktion versorgt wurden. Die Implantate durften am gleichen Tag durch eine Deckprothese funktionell belastet werden. Die 42 Patienten der Kontrollgruppe erhielten die gleiche Versorgung, jedoch wurden sie nach dem klassischen zweiphasigen Behandlungskonzept behandelt. In beiden Gruppen wurde nach fünf Jahren eine Implantatüberlebensrate von über $98 \%$ festgestellt. Grundsätzlich wurde das Behandlungskonzept als berechenbar und zuverlässig eingestuft und führte bei allen Patienten zu einer bedeutend besseren Lebensqualität. 


\subsubsection{Patientenzufriedenheit und Lebensqualität}

Bei der Erprobung neuer Therapiekonzepte nimmt die Meinung der Patienten und ihre Zufriedenheit mit der Therapie eine zentrale Stellung ein. Die Behandler haben neben den klinisch erhobenen Daten und Ergebnissen ein immer größeres Interesse, den Einfluss ihrer Therapie auf den Patienten messen zu können. Deshalb wurde in zahlreichen Studien versucht, durch die Verwendung verschiedener Fragebögen die Zufriedenheit und die orale Lebensqualität der Patienten zu erfassen. Vor allem zahnlose Patienten haben neben den funktionellen und physischen Einschränkungen durch einen herausnehmbaren Zahnersatz eine individuell stark ausgeprägte psychische Beeinträchtigung. Durch die Entwicklung spezieller Messinstrumente, wie die des OHIP (Oral Health Impact Profile) von Slade und Spencer (1994) und des MOS Short Form 36 Health Survey (SF-36) von Hays und Ware (1986), ist es gelungen, die funktionellen, psychologischen und psychosozialen Auswirkungen zu bewerten.

So verglichen Boerrigter et al. (1995) in einer Multizenterstudie die Zufriedenheit und Kaufähigkeit bei 30 Patienten mit Totalprothesen, Totalprothesen mit zusätzlicher Vestibulumplastik und implantat-retinierten Deckprothesen. Zur Befragung der Patienten wurde ein Fragebogen benutzt, der die Zufriedenheit der Patienten mit der Ästhetik, Phonetik und Kaufunktion und ihrer Prothese untersuchte. Nach der Behandlung konnte eine deutliche Verbesserung der Kaufunktion und Zufriedenheit mit den neuen Prothesen festgestellt werden. Zwischen der Implantat-Gruppe und der mit einer Vestibulumplastik konnten allerdings keine signifikanten Unterschiede festgestellt werden.

Awad und Feine (1998) konnten in einer früheren Arbeit ähnliche Ergebnisse feststellen. Sie verglichen ebenfalls implantat-retinierte Deckprothesen mit Totalprothesen. Auch hier wurde eine größere Zufriedenheit der Patienten mit implantat-retinierten Prothesen festgestellt.

Allen et al. (2001) versuchten ebenfalls die Auswirkungen implantat-gestützter Deckprothesen und konventioneller Totalprothesen auf die Lebensqualität zahnloser Patienten zu messen. Außerdem sollte der Einfluss, der präoperativen Erwartungen der Patienten auf ihre spätere Bewertung des Behandlungsergebnisses und ihrer Lebensqualität untersucht werden. Dafür wurde das Patientenkollektiv in drei Gruppen unterteilt. Alle Patienten beantworteten vor Beginn der Therapie einen OHIP-Fragebogen und einen validierten Fragebogen zur Zufriedenheit mit ihrer Totalprothese. Anschließend erhielten zwei Gruppen neue Totalprothesen (TPG1, TPG2) und eine Gruppe implantat-retinierte Deckprothesen (IG). Nach der Therapie wurde erneut eine Befragung der Patienten durchgeführt. Die Auswertung zeigte, dass die Patienten aller drei Gruppen vor der Behandlung mit ihrer Prothese unzufrieden waren. Zwei Patientengruppen wünschten sich Implantate (TPG1, IG) und stellten hohe Erwartungen an eine implantat-gestütze Deckprothese. Eine Patientengruppe favorisierte nur eine Neuanfer- 
tigung ihrer Totalprothese (TPG2). Nach der Behandlung wurde in allen drei Gruppen eine größere Zufriedenheit und verbesserte Lebensqualität festgestellt. Die Patienten, die ihre gewünschte Behandlung erhalten hatten (TPG2, IG), berichteten von einer wesentlich stärkeren Verbesserung ihrer Lebensqualität, als die Patienten, die trotz Implantatwunsch nur eine Neuanfertigung ihrer Prothese erhalten hatten (TPG1). Zusammenfassend konnte festgestellt werden, dass die Bewertung der Zufriedenheit und Lebensqualität stark durch die eigenen Erwartungen der Patienten beeinflusst wurden.

Assunção et al. (2007) verglichen in einer Studie den Einfluss implantat-retinierter Deckprothesen und konventioneller Totalprothesen bei älteren zahnlosen Patienten auf die Lebensqualität und Zufriedenheit der Patienten. 17 Patienten erhielten eine Totalprothese und weitere 17 Patienten wurden mit implantatgestützten Deckprothesen versehen. Zwei Monate nach der Integration der Prothese wurden die Patienten gebeten, einen Fragebogen auf der Basis des OHIP und OHRQL auszufüllen. Es konnten in Bezug auf Komfort, Ästhetik, Kaufunktion, Schmerzen, Phonetik und psychologische Beeinträchtigungen keine statistisch signifikanten Unterschiede zwischen den beiden Patientengruppen festgestellt werden. Die Stabilität der Prothese war jedoch in der Implantat-Gruppe signifikant höher. Insgesamt wurde die Zufriedenheit und Lebensqualität der Patienten in beiden Gruppen als gleichwertig eingeschätzt. Ursache für dieses Ergebnisse seien vor allem die exzellenten funktionellen und physikalischen Eigenschaften der konventionellen Totalprothesen gewesen.

In einer weiteren Arbeit lieferten Assunção et al. (2009) eine Literaturübersicht über Studien, die die Patientenzufriedenheit bei implantatretinierten und konventionellen Prothesen im Unterkiefer verglichen. Es wurde eine Suche aller englischsprachigen Studien bis 2008 auf Medline durchgeführt. Dabei wurden randomisierten klinische Studien und prospektive Studien favorisiert. 27 Artikel erfüllten alle Auswahlkriterien und wurden für die weitere Auswertung verwendet. Insgesamt konnte in fast allen Studien eine höhere Lebensqualität und Patientenzufriedenheit bei implantat-retinierten Deckprothesen festgestellt werden.

Siadat et al. (2008) untersuchten in einer retrospektiven Studie die Patientenzufriedenheit mit implantat-retinierten Deckprothesen im Unterkiefer. Dabei sollten vor allem die Auswirkungen des Alters, Geschlechts und der prothetischen Vorgeschichte der Patienten auf ihre Zufriedenheit analysiert werden. 55 Patienten (Durchschnittsalter 65 Jahre) erhielten im Zeitraum von 1998 bis 2004 zwei interforaminale Implantate. Anschließend wurden diese durch einen Steg verblockt und nach einer Einheilungszeit von drei Monaten mit einer Deckprothese belastet. Nach einer mittleren Tragezeit von drei Jahren erfolgte eine Befragung der Patienten. Der Fragebogen wurde speziell für die Studie entwickelt und beinhaltete die Aspekte Ästhetik, Komfort, Funktion und Hygienefähigkeit. Die Auswertung der Fragebögen zeigte, dass Patienten höheren Alters 
eine größere Zufriedenheit bezüglich der Ästhetik und des Komforts mit ihrer Prothese angaben. Außerdem berichteten Patienten, die längere Zeit zahnlos waren, die größte Zufriedenheit mit dem Behandlungsergebnis. Patienten, die vor der Behandlung schon mehrere Prothesen getragen hatten, bewerteten das Ergebnis hinsichtlich der Funktion und des Komforts am schlechtesten.

Ellis et al. (2009) betrachteten die Patientenzufriedenheit bei implantatgestützten Deckprothesen mit Kugelattachments und Magnetattachments im Unterkiefer. Das Patientenkollektiv umfasste 16 Patienten, die in zwei Gruppen unterteilt wurden. Beide Gruppen wurden mit interforaminalen Implantaten versorgt. Anschließend erhielt eine Gruppe als Retentionselement Kugelattachments und die andere Magnetattachments. Die Patienten wurden gebeten, drei Monate vor und sechs Monate nach der Behandlung einen Fragebogen zur Patientenzufriedenheit nach Mc Gill zu beantworten. Die Ergebnisse zeigten bei beiden Retentionssystemen eine deutliche Verbesserung der Patientenzufriedenheit. Insgesamt konnten bei den Patienten mit Kugelattachments höhere Zufriedenheitswerte als bei denen mit Magnetattachments festgestellt werden.

In einer Arbeit von Alfadda et al. (2009) wurde der Einfluss der Sofortbelastung und des klassischen Belastungskonzepts auf die Lebensqualität und Zufriedenheit der Patienten untersucht. In der Testgruppe befanden sich 35 Patienten, die mit jeweils zwei Implantaten in Regio 033, 043 und einer konfektionierten Stegkonstruktion versorgt wurden. Diese durften am gleichen Tag durch eine Deckprothese funktionell belastet werden. Die 42 Patienten der Kontrollgruppe erhielten die gleiche Versorgung, jedoch wurden sie nach dem klassischen zweiphasigen Behandlungskonzept behandelt. Vor und nach der Behandlung beantworteten die Patienten das Oral Health Impact Profile. Nach der Behandlung konnte in beiden Gruppen eine deutliche Verbesserung der Lebensqualität festgestellt werden. Zwischen der Test- und Kontrollgruppe ergaben sich jedoch hinsichtlich der Lebensqualität keine statistisch signifikanten Unterschiede. Insgesamt führte das Behandlungskonzept bei allen Patienten zu einer bedeutend besseren Lebensqualität.

\subsection{Das Konzept der Satellitenimplantate}

Das Konzept der Satellitenimplantate basiert auf dem aus der Traumatologie entlehnten Mikroosteosyntheseverfahren, das zur Rekonstruktion alveolärer Defekte mit anschließender Implantation entwickelt wurde. Bei der alveolären Rekonstruktion mit anschließender Implantation wird eine Verbindung zwischen Implantat, Osteosyntheseplatte und Knochen hergestellt, so dass es auch unter Belastung der Implantate und des augmentierten Knochens durch eine Deckprothese zur Osseointegration kommt (Engelke et al. (1997)). 
Dieses Konzept der subgingivalen Stabilisation eines Implantats durch eine Mikroosteosyntheseplatte wurde weiterentwickelt und führte schließlich zum Konzept der Satellitenimplantation. Ziel dieser Entwicklung ist es, durch die Verwendung von zusätzlichen „Hilfsimplantaten“ das eigentliche „Zentralimplantat“ so zu stabilisieren, dass eine sofortige okklusionsbezogene Belastung ermöglicht werden kann. Das eigentlichen Implantat wird durch die kortikale Verankerung einer oder mehrere Osteosyntheseschrauben im Alveolarknochen zusätzlich fixiert. Die Verbindung der Osteosyntheseschrauben zum Implantat wird über entsprechende subgingivale Konnektoren hergestellt (siehe Abbildung 1.1a) . Auf Grund der Position und Funktion der Osteosyntheseschrauben in Bezug auf das Zentralimplantat werden diese als "Satellitenimplantate“ bezeichnet. Der subgingivale Verbund des Implantats mit den kortikalen Osteosyntheseschrauben ähnelt dem bereits bekannten Prinzip der primären Verblockung von Implantaten durch supragingivale Stegkonstruktionen, die eine Sofortbelastung ermöglichen und in zahlreichen Studien erfolgreiche Resultate hervorgebracht haben (Ledermann (1979), Degidi et al. (2007), Stoker und Wismeijer (2008)).

Meyer et al. (2004) zeigten, dass nicht die sofortige Belastung an sich die Osseointegration entscheidend beeinflusst, sondern das Ausmaß an Mikrobewegungen im Interface zwischen Implantat und Knochen während der Einheilphase ausschlaggebend ist. Somit ist das wichtigste Kriterium für eine erfolgreiche Osseointegration eine gute Primärstabilität nach der Implantation. Durch die zusätzliche Verankerung von Osteosyntheseschrauben in der Kortikalis werden die Mikrobewegungen verringert und dadurch die Primärstabilität des Implantats deutlich erhöht.

Engelke et al. (2005) veröffentlichten Ergebnisse einer prospektiven Studie, an der 20 Patienten teilnahmen (15 Frauen und 5 Männer). Dabei wurden jedem Patienten zwei Implantate in der Regio interforaminalis inseriert, die anschließend mit einer Deckprothese versorgt und sofort belastet wurden. Nach einem Beobachtungszeitraum von 10 Monaten konnte bei allen Implantaten eine erfolgreiche Osseointegration festgestellt werden und die Erfolgsrate lag bei $100 \%$.

Weitere Ergebnisse dieser prospektiven Studie ergaben bei der Nachuntersuchung von 92 Patienten nach einem Beobachtungszeitraum von 22 Monaten, eine Überlebensrate von 97,97 \%. Die Erfolgsrate nach Jahn und d'Hoedt (1992) und Naert et al. (1992), Snauwaert et al. (2000) betrug 97,83 \% bzw. 98,37 \%. Von den 182 inserierten Implantaten kam es zu drei Implantatverlusten. 


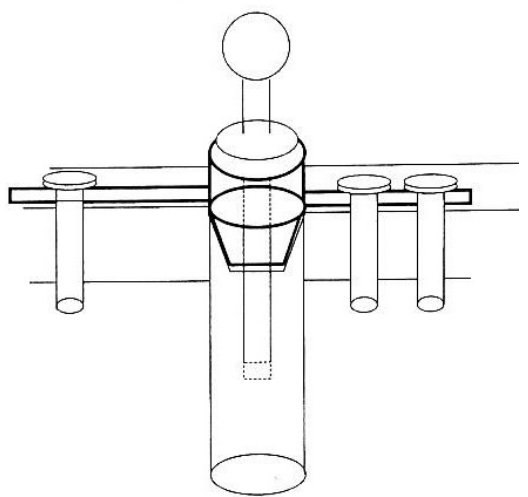

(a) Prinzip der subgingivalen Satellitenimplante

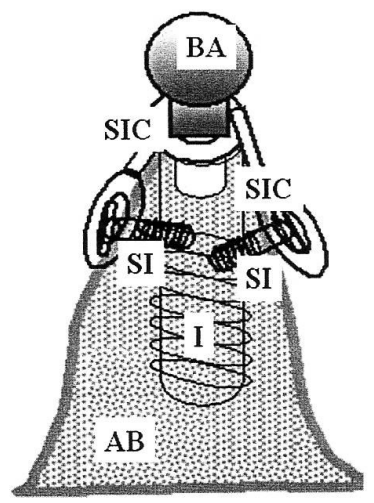

(b) 1b Schematische Darstellung der Satellitenimplantate
Implantat

Kugelattachment

Satellitenimplantkonnektor

Satellitenimplantat

Alveolarknochen

Abbildung 1.1: Schematische Darstellung der Satellitenimplantate

\subsection{Zielsetzung der Arbeit}

Im Rahmen dieser prospektiven Studie soll der Erfolg des Konzepts der Sofortbelastung zweier interforaminaler Implantate mit einer Deckprothese im Unterkiefer unter Verwendung kortikaler Satellitenimplantate im Beobachtungzeitraum von 2001-2009 bewertet werden. Dabei sollen die beiden verwendeten Implantatsysteme hinsichtlich der folgenden Parameter evaluiert und verglichen werden:

- Überlebensrate und Erfolgsquote der sofortbelasteten Implantate

- Beurteilung der Implantatstabilität durch Bestimmung der Periotestwerte

- Messung des periimplantären horizontalen Knochenabbaus

- Zustand der periimplantären Gingiva

- Darstellung eventueller Komplikationen und Probleme.

Des Weiteren soll durch eine Befragung der Patienten ihre Zufriedenheit mit der Behandlung sowie ihre mundbezogene Lebensqualität untersucht werden. 


\section{Material und Methode}

\subsection{Patientenkollektiv}

Im Zeitraum von 2001 bis 2005 wurden 86 Patienten (54 Frauen und 32 Männer) für diese prospektive Studie ausgewählt. Das durchschnittliche Alter der Patienten betrug zum Zeitpunkt der Implantation 64 Jahre. Jeder Patient wurden mit zwei enossalen Implantaten nach dem Göttinger-Konzept der kortikalen Mikrofixation in der interforaminalen Region versorgt. Somit wurden insgesamt 172 Implantate (davon 68 Semadosimplantate, 104 Xiveimplantate) inseriert. Die Durchführung der Implantation fand in der zahnärztliche Chirurgie an der Universitätsklinik Göttingen statt.

Das Patientenkollektiv wurde mittels der folgenden Kriterien ausgewählt:

Einschlusskriterien :

- Alle Patienten, bei denen eine Indikation für die Versorgung mit dentalen Implantaten im zahnlosen Kiefer bestand

- abgeschlossenes Längenwachstum, ein uneingeschränkter guter Allgemeinzustand und ein qualitativ wie quantitativ ausreichendes knöchernes Implantatlager

Ausschlusskriterien:

- Patienten mit psychogener Prothesenintoleranz

- Patienten mit allgemeinmedizinischen Risiken, die eine Lokalanästhesie und/ oder einen zahnärztlichen chirurgischen Eingriff von ca. 1 Stunde Dauer ausschließen

- Patienten mit Alveolarkammatrophien der Klassen D4 nach Lekholm und Zarb (1985) respektive der Klasse I nach ASCIi

- Bei Patienten mit Drogenabusus

- Bei Rauchern mit einem regelmäßigen Konsum von mehr als 10 Zigaretten pro Tag

- Patienten mit akuten entzündlichen Prozessen in der Mundhöhle

- Ausgangssituationen, bei denen der implantologische Eingriff die Kieferbasisstabilität gefährden könnte

- Patienten mit unbehandelter Parodontitis. 


\subsection{Versorgung der Patienten}

\subsubsection{Implantatsysteme}

Im Rahmen dieser Studie wurden das Semados Implantat der Firma Bego Bremen/ Deutschland und das Xive Implantat der Firma Friadent/ Mannheim/ Deutschland verwendet.

Bei beiden Implantatsystem handelt es sich um Schraubimplantate aus Reintitan. Das Semadosimplantat hat eine konische Grundform mit einer mikrostrukturierten, osteokonduktiven Oberfläche. Dabei nimmt die Gewindetiefe von der Implantatschulter zum apikalen Ende aufgrund der Konizität des Schafts zu. Die Implantataufbauverbindung besteht aus einem Innenkonus und Sechskant.

Das Xiveimplantat hat einen annähernd zylindrischen Grundkörper. Krestal befindet sich ein Kondensationsgewinde und apikal ein selbstschneidendes Gewinde. Die Oberfläche des Implantats hat eine geätzte und gestrahlte Tiefenstruktur. Die Verbindung von Implantat zu Abutment wird durch einen Innensechskant hergestellt.

Jeder Patient erhielt in dieser Studie zwei Implantate in der Regio interforaminalis. Demzufolge wurden insgesamt 172 Implantate inseriert (davon 68 Semadosimplantate, 104 Xiveimplantate). In der Semadosgruppe wurden Implantate der Längen $10 \mathrm{~mm}$, $13 \mathrm{~mm}, 15 \mathrm{~mm}$ und $18 \mathrm{~mm}$ verwendet. Der Implantatdurchmesser betrug 3,25mm und 3,75 mm. In der Xivegruppe betrugen die Implantatlängen 9,5 mm $11 \mathrm{~mm}, 13 \mathrm{~mm}, 15$ mm und $18 \mathrm{~mm}$. Die Implantatdurchmesser waren 3,4 $\mathrm{mm}$ und 3,8 $\mathrm{mm}$ breit.

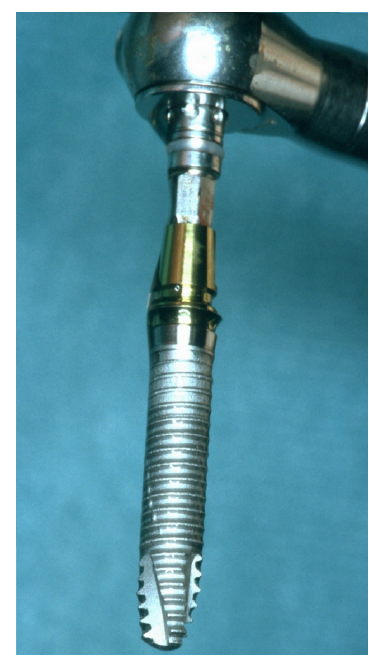

(a) Xiveimplantat

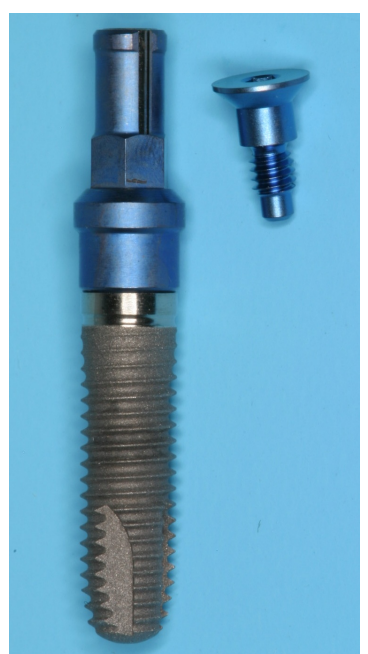

(b) Semadosimplantat

Abbildung 2.1: Implantatsysteme 


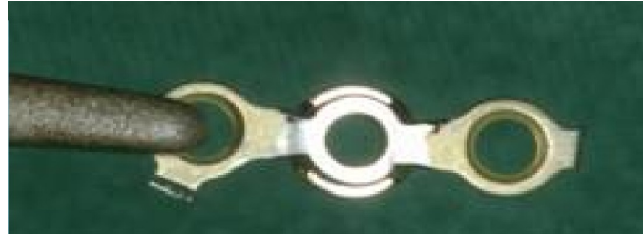

(a) Satellitenkonnektor

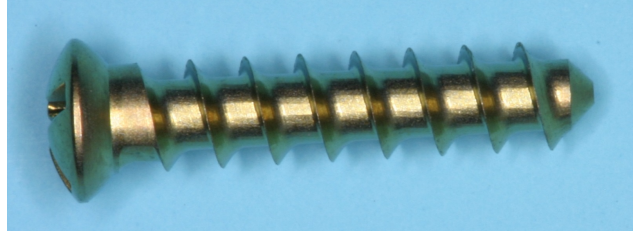

(b) Satellitenimplantat

Abbildung 2.2: Satellitenimplantat und Konnektor

\subsubsection{Das Operationsverfahren}

Die chirurgischen Eingriffe wurden im Operationssaal der ZMK des Göttinger Universitätsklinikums in Lokalanästhesie (Ultracain Dental forte, Hoechst AG, Frankfurt am Main/Deutschland) durchgeführt.

Nach krestaler Inzision in Kombination mit mesiovestibulärer Entlastung und Abpräparation von Mukoperiostlappen, wurden die Positionierung und Achsenrichtung der Implantation mit Hilfe der Prothese als Operationsschablone nach prothetischen Kriterien umgesetzt. Die Prothese wies Perforationen auf den okklusalen Flächen der Canini auf. Die Unregelmäßigkeiten der krestalen Alveolarknochen wurden ausgeglichen, und der Alveolarknochen wurde horizontal reduziert, so dass eine Insertion mit einem 3,25 mm Implantatdurchmesser möglich war. Die approximalen Knochenbereiche wurden an das Niveau des Implantats angepasst, um die Fixierung der Satellitenimplantate zu vereinfachen.

Die Aufbereitung der Implantatlager erfolgte mit den Bohrern analog den Richtlinien des Herstellers. Auffällige Befunde wurden mit dem Endoskop (Karl Storz GmbH \& Co. KG, Tuttlingen Deutschland) inspiziert. Beim überwiegend zervikalen Alveolarknochen der Klasse 1 nach Lekholm und Zarb (1985) wurde mit einem Kopfsenker vor der Insertion der Implantate aufbereitet. Beim Knochen der Klasse 2 und 3 nach Lekholm und Zarb (1985) wurden keine Kopfsenker verwendet. Die Zentralimplantate wurden juxtaalveolär auf dem Niveau des krestalen Knochen inseriert. Die Satellitenimplantatkonnektoren wurden an die alveoläre Knochenform individuell angepasst. Nach der Aufbereitung mit der 1,5mm (Knochen Kl. I nach Lekholm und Zarb (1985)) bzw. 1,2 mm (Knochen Kl. 2 nach Lekholm und Zarb (1985)) kortikalen Lanzette wurden die Satellitenimplantate inseriert. Die Insertion wurde unter ständiger Spülung von physiologischer Kochsalzlösung durchgeführt.

Postoperativ erfolgte der Wundverschluss mit Einzelknopfnähten, und die Anfertigung eines Orthopantomogramm. Unmittelbar nach der Operation wurde die prothetische Versorgung mit der abnehmbaren Kugelattachmentprothese vorgenommen. In den Prothesen wurden die Matrizen für die Kugelattachments mit einem Prothesenkunststoff 
(Paladur, Heraeus Kulzer GmbH, Hanau, Deutschland) fixiert. Die Okklusionskontakte wurden kontrolliert, und der Prothesenrand wurde in den implantierten Regionen ausreichend abgetragen.

Abschließend erhielten die Patienten eine Hygieneunterweisung. Zudem wurde ihnen eine 0,1 \%ige Chlorhexidinlösung (Chlorhexamed 0,1 \% Blendax, Mainz) und ein nichtsteroidales Antirheumatikum (Paracetamol, Hexal AG, Holzkirchen, Deutschland) verschrieben. Des Weiteren wurden sie gebeten, während der ersten zwei Tage die Prothese nicht zu entfernen und Vorsichtsmaßnahmen, wie das Vermeiden von Nikotin-, Alkohol-, Koffeinkonsum und körperlicher Anstrengung, einzuhalten. Der nächste Kontrolltermin erfolgte zwei Tage später. Es fand eine Kontrolle der Okklusion und Retention der Prothese statt. Nach sieben Tagen konnten die Fäden entfernt werden, und 14 Tage post operationem wurden die Prothesen unterfüttert und die Matrizen aktiviert. Drei Monate nach dem chirurgischen Eingriff wurden bei allen Patienten die Satellitenimplantate unter Lokalanästhesie entfernt.

\subsection{Evaluationen}

\subsubsection{Verweildaueranalyse und Erfolgskriterien}

Als statistisches Standardverfahren zur zeitabhängigen Untersuchung des Parameters Implantatverlust wurde die Überlebenszeitanalyse nach Kaplan und Meier (1958) verwendet. Entscheidend ist bei dieser Methode, dass es keine fest vorgegeben Beobachtungsintervalle gibt, sondern die Ereignisse (Implantatverluste) den Beobachtungszeitraum definieren. Folglich beginnt mit jedem Implantatverlust ein neues Zeitintervall. Für jedes Implantat, das ein Zeitintervall überlebt hat und sich im nächsten Intervall befindet, wird die Überlebenswahrscheinlichkeit berechnet. Dadurch kommt der Liegedauer und der unter Risiko stehenden Anzahl an Implantaten eine besondere Gewichtung zu.

Um außerdem den Zustand der periimplantären Weich- und Hartgewebsparameter mit zu berücksichtigen, wurden die Erfolgskriterien nach Jahn und d'Hoedt (1992) und Naert et al. (1992)/ Snauwaert et al. (2000) hinzugezogen (siehe Tabelle 2.2). Des Weiteren sollten mögliche Faktoren, die einen Einfluss auf die Überlebensrate der Implantate haben könnten berücksichtigt werden. Als mögliche Einflussfaktoren wurden die zwei verwendeten Implantatsysteme, das Alter der Patienten sowie die Position im Kiefer (Regio 33 und 43) angenommen. Diese verschiedenen Faktoren sind voneinander abhängig. Dieser Abhängigkeit sollte durch eine adjustierte Varianzschätzung im Cox Regressionsmodell Rechnung getragen werden. 


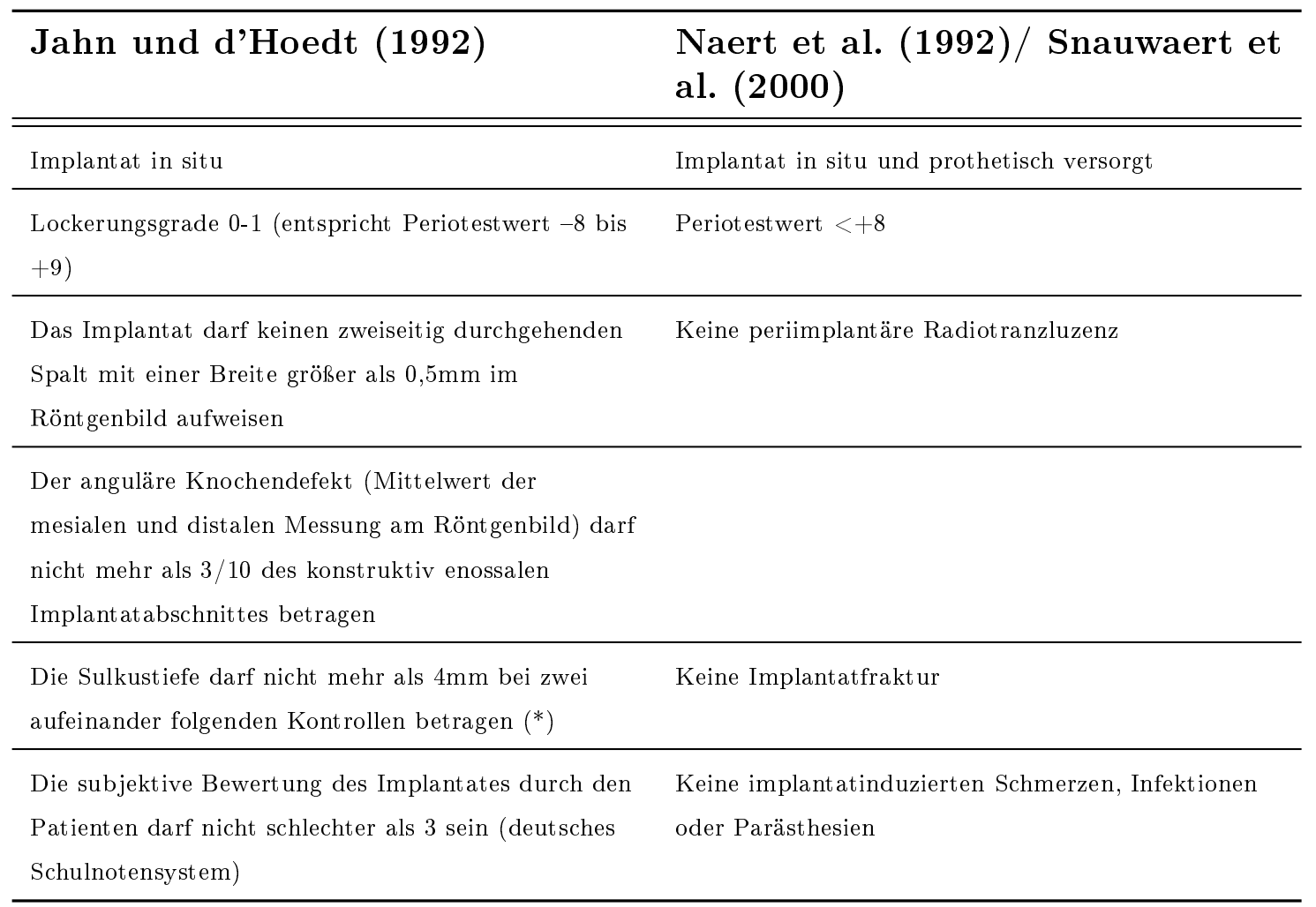

Tabelle 2.2: International verwendete Erfolgskriterien für enossale dentale Implantate

\subsubsection{Beurteilung der periimplantären Gingiva}

Um den Zustand der periimplantären Gingiva beurteilen zu können, wurde mit einer kalibrierten Parodontalsonde (HuFriedy) die Taschentiefen mesial und distal der Implantate vom Margo gingivae bis zum tiefsten Punkt der periimplantäre Tasche bestimmt. Die Messwerte wurden dann im Göttinger Untersuchungsprotokoll in Millimetern dokumentiert. Außerdem wurde beim Sondieren der perimplantären Gingiva auf auftretende Blutungen geachtet und als positiv oder negativ im Messprotokoll vermerkt.

\subsubsection{Das Periotestgerät}

Der Periotest (Medizintechnik Gulden, Bensheim) ist ein nicht invasives, elektromechanisches Messverfahren zur Bestimmung der Primärstabilität und Osseointegration von Implantaten. Außerdem wird es auch zur Diagnose von Parodonthopathien und Beurteilung okklusaler Belastung eingesetzt. Es wurde von W. Schulte an der Universität in Tübingen entwickelt und von d'Hoedt et al. (1985) beschrieben. 
Beim Periotestgerät perkutiert ein elektrisch angetriebener und elektronisch überwachter Metallstößel mit einem Gewicht von $8 \mathrm{~g}$ viermal pro Sekunde bei konstanter Geschwindigkeit gegen den zu überprüfenden Körper. Der Messvorgang dauert vier Sekunden, sodass das Messobjekt 16 mal perkutiert wird. Durch den Kontakt mit der Oberfläche wird der Stößel gebremst. Je fester das Implantat/Zahn im Knochen verankert ist, desto größer ist auch die Bremswirkung und so-

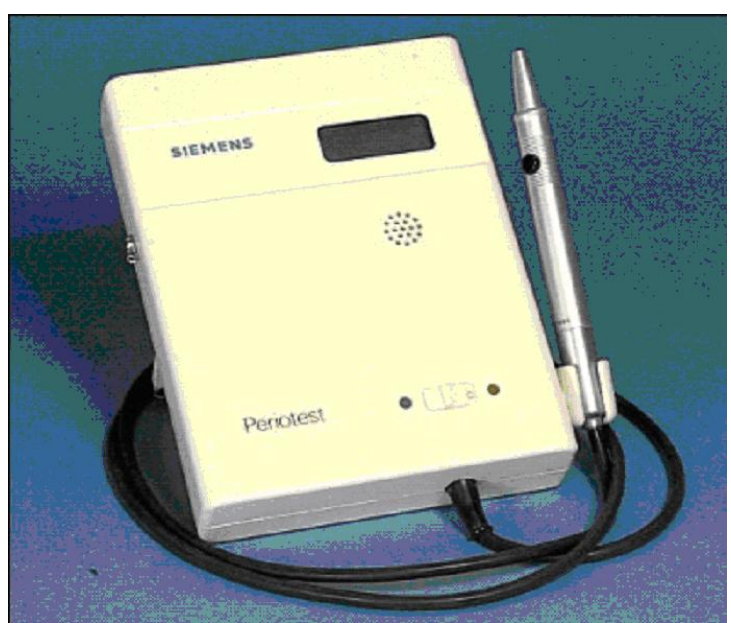

Abbildung 2.3: Periotestgerät mit der Dämpfungseffekt des umgebenden Knochengewebes auf den Metallstößel (Olivé und Aparicio (1990)). Nachdem der Stößel die zu prüfende Stelle berührt hat, prallt er von ihr zurück. Je schneller der Stößel dies tut, desto größer ist auch die Dämpfung. Das Periotestgerät misst also die Kontaktzeit des Metallstößels an der Oberfläche des Implantats/Zahnes. Diese liegt im Bereich von einer Millisekunde. Durch eine Umrechnung gibt das Gerät jedoch nicht einen Wert im Bereich einer Millisekunde an, sondern einen Wert auf einer Skala von -8 bis +50 . Dabei stellen negative Werte eine hohe und positive eine geringe Stabilität des Implantats/Zahnes dar.

\subsubsection{Bestimmung der Periotestwerte}

Die Periotestwerte wurden möglichst horizontal zum Implantat bestimmt. Außerdem wurde darauf geachtet, dass der korrekte Messabstand zum Implantat eingehalten wird. Die Messwerte wurden ebenfalls in das Untersuchungsprotokoll eingetragen.

\subsubsection{Bestimmung des periimplantären Knochenabbaus}

Zur Beurteilung des horizontalen Knochenabbaus wurden Orthopantomogramme mit dem Gerät "Orthophos" der Firma Siemens (Bensheim, Deutschland) angefertigt. Der eventuelle Knochenabbau wurde nach einem mittleren Untersuchungszeitraum von 52 Monaten nach der Insertion der Implantate vermessen. Dabei wurden eindeutige Referenzpunkte festgelegt, die gut im Röntgenbild identifizierbar waren und eine leichte Reproduzierbarkeit der Messung ermöglichten. Ein Referenzpunkt wurde an der Implantatschulter, der zweite am tiefsten Punkt des horizontalen Knochenabbaus festgelegt. Damit der Knochenabbau möglichst präzise bestimmt werden konnte, wurde ein digitales Vermessungsverfahren der Panoramaschichtaufnahmen gewählt. Dafür mussten die Röntgenbilder mit dem Scanner Linotype digitalisiert werden. Anschließend 
wurden die digitalisierten Aufnahmen in das Programm Sidexis XG der Firma Sirona importiert. Um die Knochenkonturen besser beurteilen zu können, wurde in dem Programm eine Graustufenfeinabstimmung der Aufnahmen vorgenommen (siehe Abbildung 2.4). Die Panoramaschichtaufnahmen unterliegen einem Vergrößerungsfaktor von 1,25, sodass vor der eigentlichen Messung eine Längenkalibrierung durchgeführt werden musste. Dafür wurde die digital dargestellte Länge der Implantate mit dem Cursor markiert (siehe Abbildung 2.5) und auf die tatsächliche bekannte Implantatlänge kalibriert (siehe Abbildung 2.6). Anschließend wurde die reale Länge des Implantats überprüft. Danach wurde der horizontale Knochenabbau mesial und distal an der Schulter des Implantates bestimmt (siehe Abbildung 2.7).

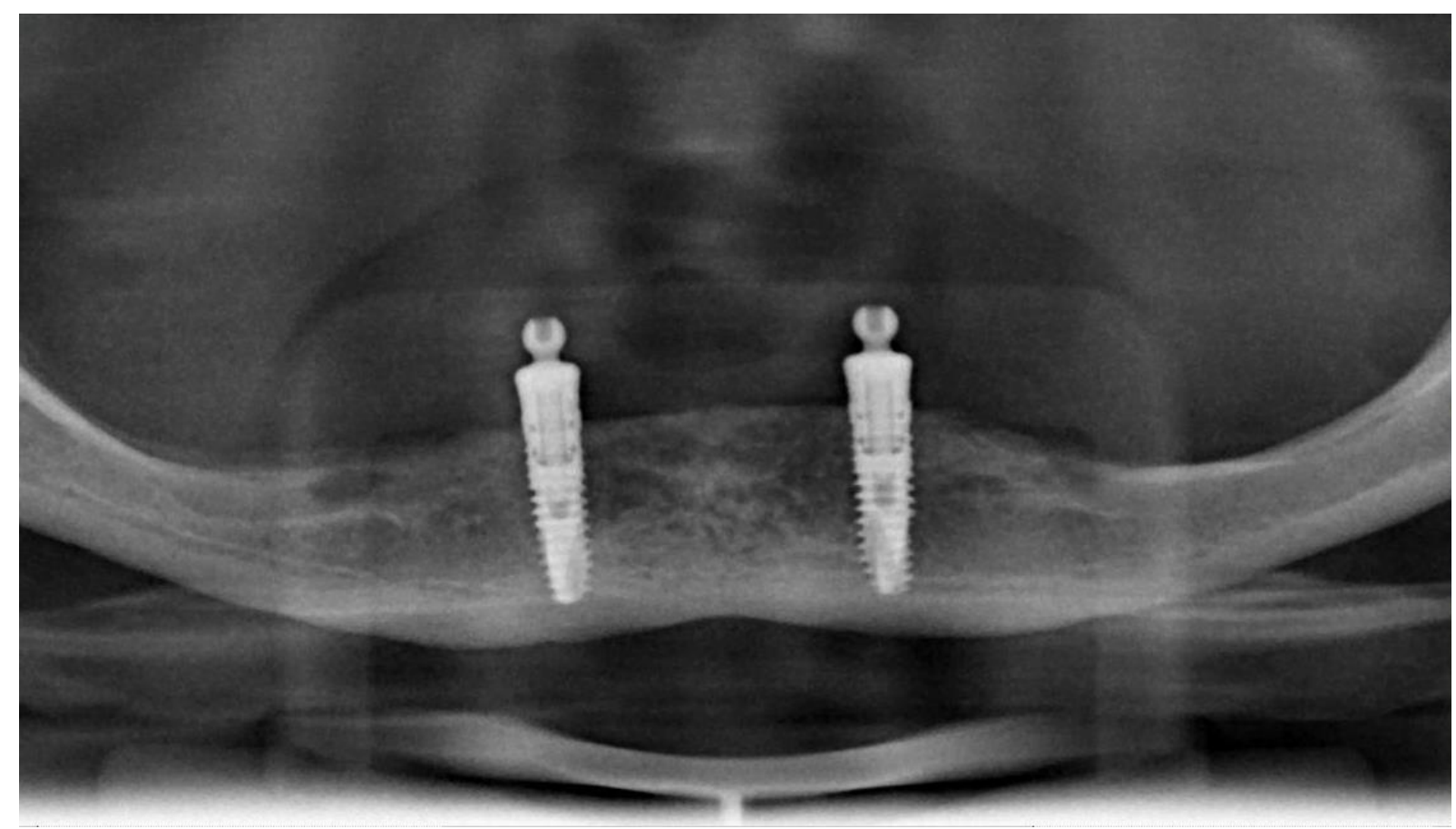

Abbildung 2.4: Digitalisierte OPAN-Aufnahme in Sidexis-Programm importiert 


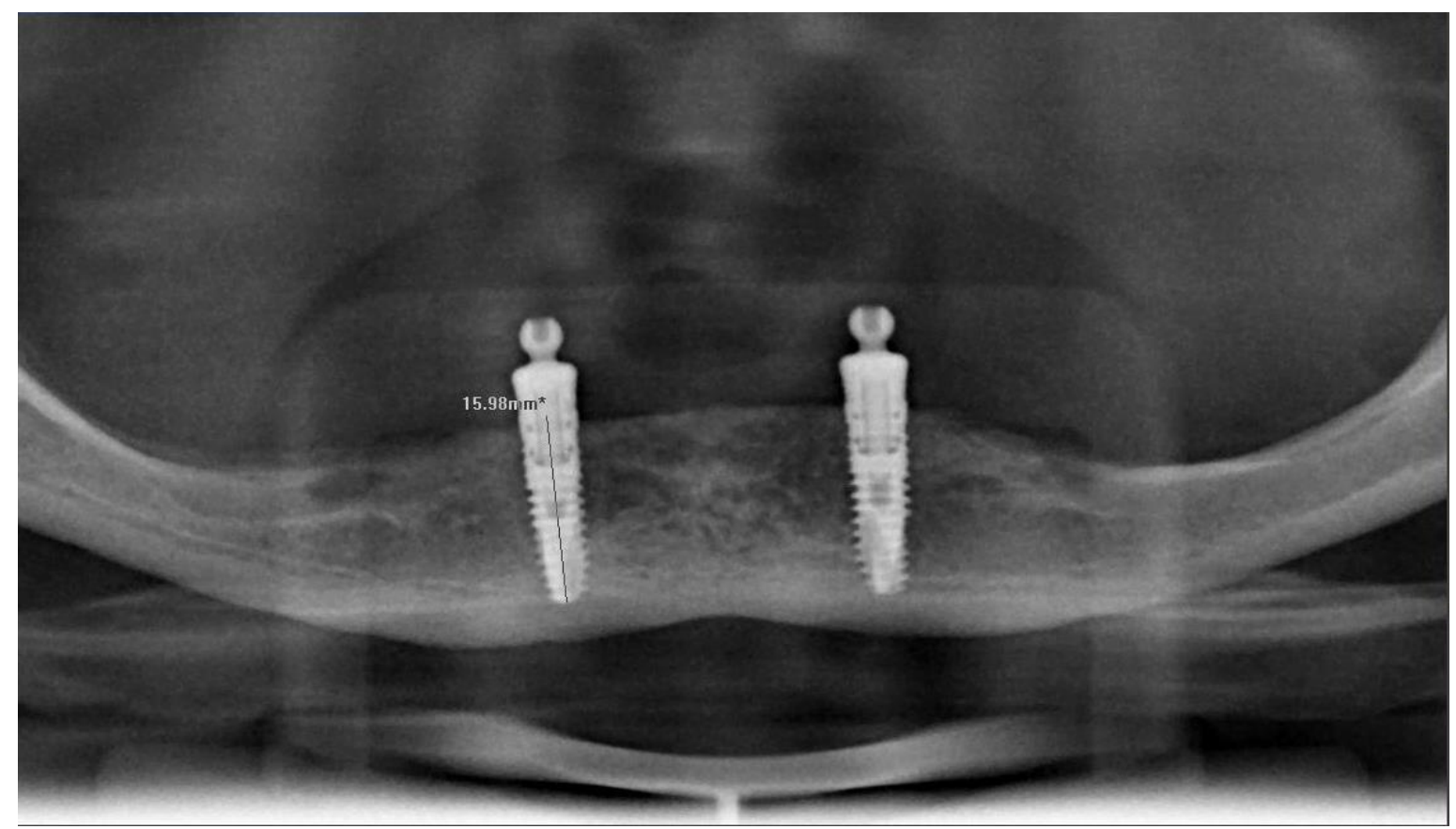

Abbildung 2.5: Längenmessung des Implantates vor Kalibrierung

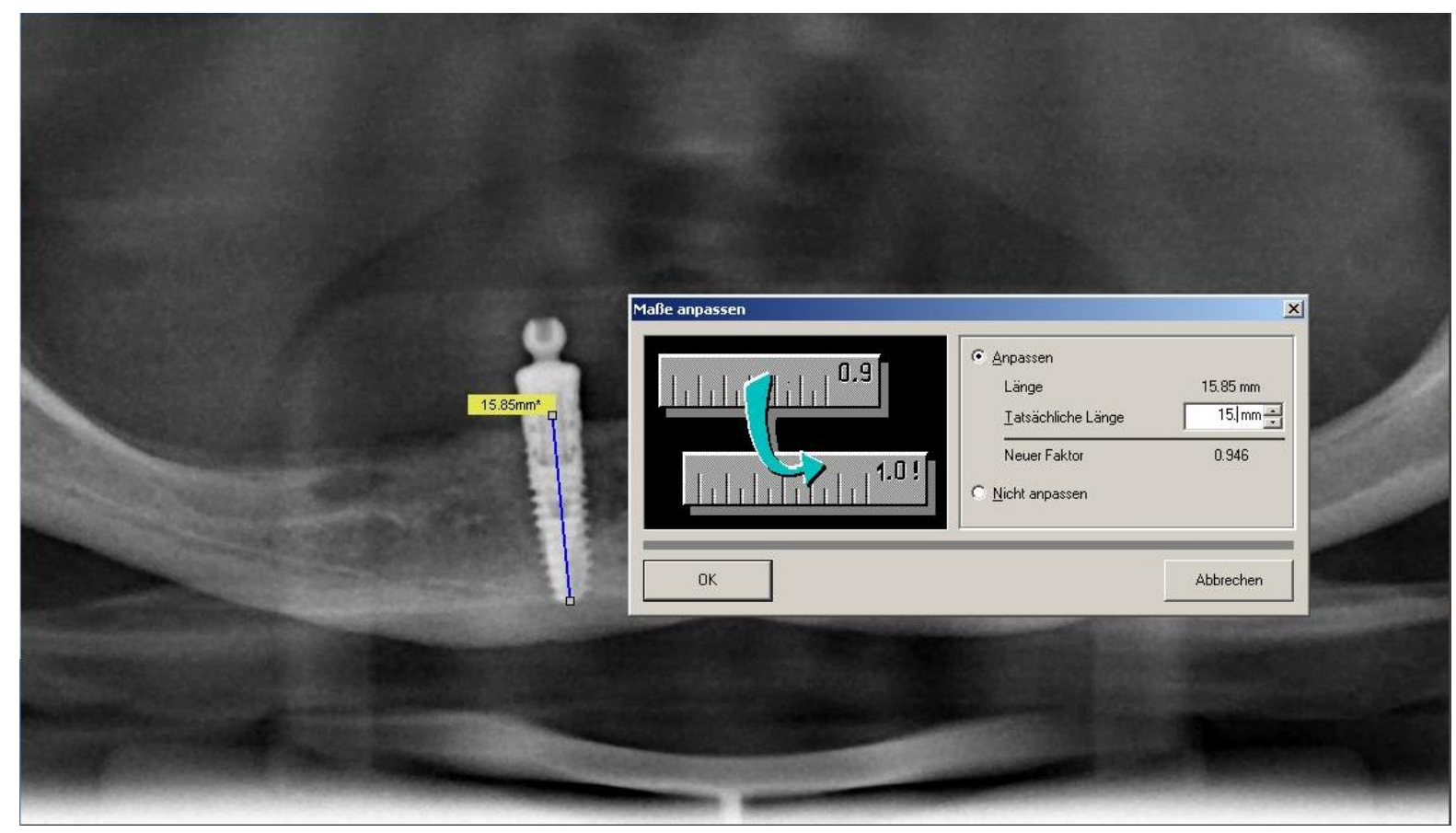

Abbildung 2.6: Kalibrieren der Längenmessung 


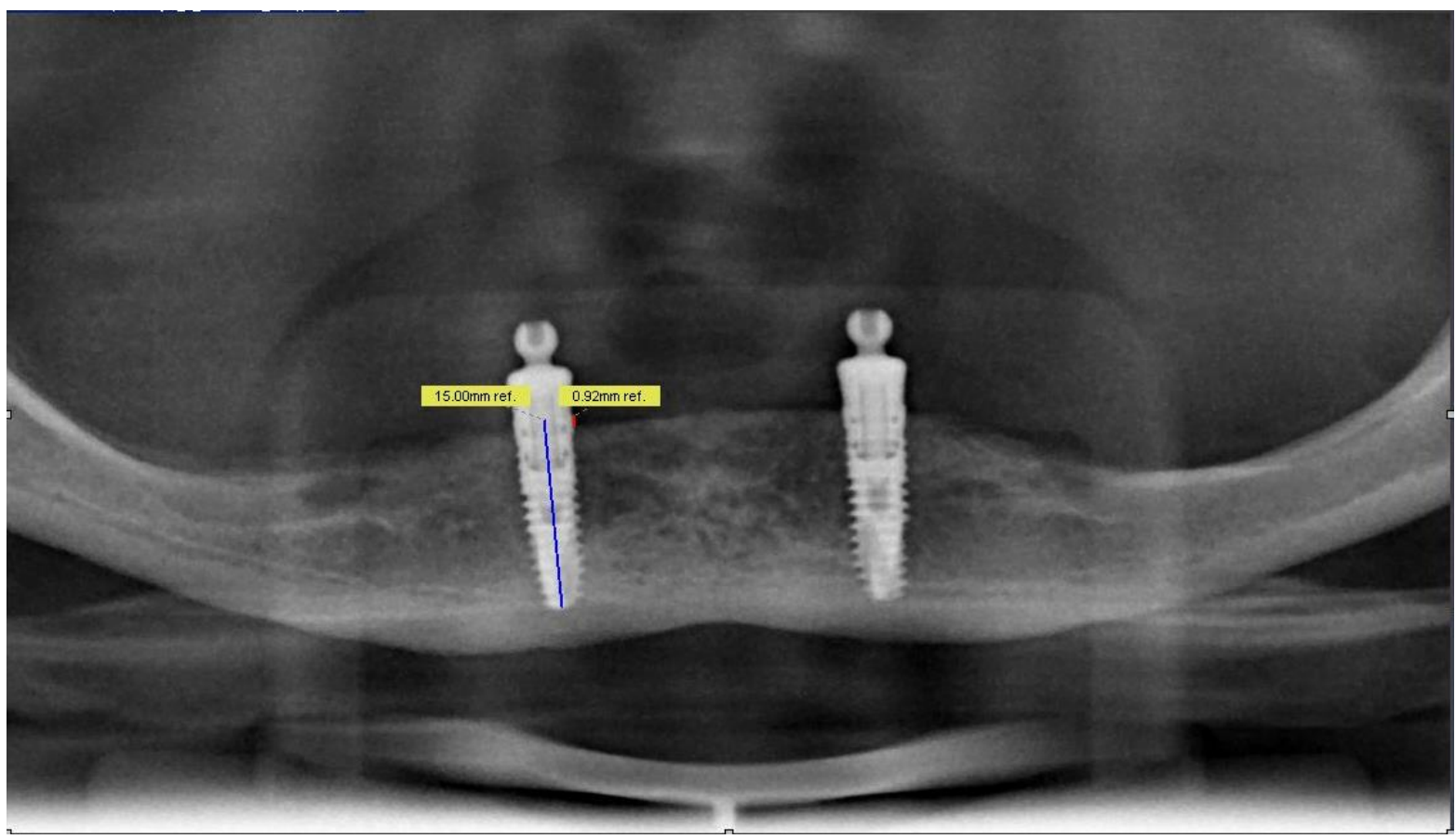

Abbildung 2.7: Ausmessen des horizontalen Knochenabbaus

\subsubsection{Patientenfragebögen}

Zur Erfassung der Patientenzufriedenheit und oralen Lebensqualität wurden zwei verschiedene Fragebögen verwendet. Die Patientenzufriedenheit wurde mit einem speziell für die Studie entwickelten Fragebogen bestimmt. Dieser bestand aus sieben Fragen, wobei vier durch eine Benotung nach dem deutschen Schulnotensystem und 2 durch Ja/Nein beantwortet werden sollten. Eine Frage wurde offen gestellt.

1. Frage zur Zufriedenheit des Gesamtresultates: Wie beurteilen Sie das Gesamtresultat Ihrer Behandlung?

2. Frage zur Halt der Prothese: Wie schätzen Sie den Halt Ihrer Prothese ein?

3. Frage zur Kaufunktion: Wie schätzen Sie Ihre Kaufunktion insgesamt ein?

4. Frage zur Reinigung der Prothese: Haben/ hatten Sie Probleme bei der Reinigung Ihrer Implantate und/oder Prothese?

5. Frage zu Komplikationen/Problemen mit der Prothese: Wie oft mussten Sie aufgrund von Problemen oder Beschwerden mit Ihrer Prothese einen Zahnarzt aufsuchen? (bitte die Anzahl der Besuche eintragen) 
6. Frage zur Behandlungsempfehlung: Würden Sie die Behandlung einem guten Bekannten weiterempfehlen?

7. Frage zur Mundgesundheit: Sind Sie zufrieden mit Ihrer Mundgesundheit?

Zur Auswertung der oralen Lebensqualität der Patienten wurde das OHIP-20 verwendet. Dieser Fragebogen basiert auf dem OHIP (Oral Health Impact Profile), welches von Slade und Spencer (1994) in Australien entwickelt wurde. Das OHIP ist ein Frageinstrument, das speziell zur Erfassung der oralen Gesundheit und Lebensqualität eines Patienten konzipiert wurde. Dieser Fragebogen setzt sich aus sieben Subskalen zusammen (Funktionelle Einschränkung, Schmerzen, psychisches Unbehagen, physische Beeinträchtigung, psychische Beeinträchtigung, soziale Beeinträchtigung, Behinderung im Alltag), die sowohl physische also auch psychische Probleme des Patienten beleuchten. Es wurde zuvor in vielen Studien auf seine Verlässlichkeit und Validität überprüft (Slade (1998)).

Außerdem wurde es in vielen Studien zur Bewertung des Behandlungsergebnisses von zahnlosen Patienten benutzt. Da das OHIP mit 49 Fragen relativ lang ist, wurden in verschiedenen Studien kürzere Fassungen vorgeschlagen (Slade (1997), Allen und Locker (2002)). So entstand das OHIP-20, welches im Vergleich zum OHIP von Slade und Spencer (1994) nur 20 Fragen beinhaltet und speziell für zahnlose Patienten bestimmt ist. Gerade im prothetischen Bereich ist dieser Fragebogen sehr differenziert und wurde von Allen und Locker (2002) hinsichtlich seiner Validität erfolgreich überprüft. Die

\begin{tabular}{ll}
\hline Antwortoptionen & Punktwert \\
\hline \hline immer & 1 \\
\hline sehr oft & 2 \\
\hline ziemlich oft & 3 \\
\hline gelegentlich & 4 \\
\hline fast nie & 5 \\
\hline nie & 6 \\
\hline
\end{tabular}

Tabelle 2.4: Die Antwortoptionen des OHIP-20-Fragebogens (Woelk 2007) Fragen werden mit Hilfe so genannter Auswahllisten beantwortet. Dabei steht zur Auswahl, eine Frage mit „immer, sehr oft, ziemlich oft, gelegentlich, fast nie oder nie“ auf dem Fragebogen anzukreuzen (siehe Tabelle 2.4). Die zwanzig Fragen sind in sieben Dimensionen gegliedert, die sich in ihrem Blickwinkel auf die Probleme in der Mundhöhle unterscheiden (siehe Tabelle 2.6). Die Vorteile des OHIP sind die breite Anwendungsmöglichkeit in der Zahnmedizin, die variable Anwendung in der Auswertung (Allen und Locker (1997)) und die internationale Validität (Allison et al. (1999)). 


\section{Dimension}

\begin{tabular}{|c|c|}
\hline 1 & $\begin{array}{l}\text { Funktionelle Einschränkung } \\
\text { Haben Sie aufgrund von Problemen mit den Zähnen, dem Zahnersatz oder durch andere Probleme in der } \\
\text { Mundhöhle Schwierigkeiten beim Kauen bestimmter Nahrungsmittel gehabt? } \\
\text { Haben sich des Öfteren Speisereste zwischen Ihren Zähnen bzw. unter den Prothesen angesammelt? } \\
\text { Hatten Sie das Gefühl, dass Ihr Zahnersatz nicht richtig passt? }\end{array}$ \\
\hline 2 & $\begin{array}{l}\text { Schmerzen in der Mundhöhle oder an den Zähnen } \\
\text { Haben Sie unter Schmerzen in der Mundhöhle gelitten? } \\
\text { War es für Sie unangenehm, wegen Problemen mit den Zähnen, dem Zahnersatz oder anderer Probleme in } \\
\text { der Mundhöhle, bestimmte Nahrungsmittel zu essen? } \\
\text { Hatten Sie wunde Stellen im Mund? } \\
\text { Waren Ihnen Ihre Prothesen unangenehm? }\end{array}$ \\
\hline 3 & $\begin{array}{l}\text { Psychisches Unbehagen } \\
\text { Haben Ihre Probleme mit den Zähnen, dem Zahnersatz oder andere Probleme in der Mundhöhle Sie } \\
\text { beunruhigt? } \\
\text { Haben Sie sich aufgrund des Zustandes Ihrer Zähne, des Zahnersatzes oder der Mundhöhle unsicher } \\
\text { gefühlt? }\end{array}$ \\
\hline 4 & $\begin{array}{l}\text { Physische Beeinträchtigung } \\
\text { Haben Sie es aufgrund von Problemen mit den Zähnen, dem Zahnersatz oder anderer Probleme in der } \\
\text { Mundhöhle vermieden, einige Nahrungsmittel zu essen? } \\
\text { Haben Ihre Probleme mit den Zähnen, dem Zahnersatz oder andere Probleme in der Mundhöhle Ihre } \\
\text { Ernährungsgewohnheiten ungünstig beeinflusst? } \\
\text { War es Ihnen unmöglich mit Ihrem Zahnersatz zu essen? } \\
\text { Mussten Sie Ihre Mahlzeiten wegen Problemen mit den Zähnen, dem Zahnersatz oder durch andere } \\
\text { Probleme in der Mundhöhle unterbrechen? }\end{array}$ \\
\hline 5 & $\begin{array}{l}\text { Psychische Beeinträchtigung } \\
\text { Haben Sie sich über Probleme mit den Zähnen, dem Zahnersatz oder andere Probleme in der Mundhöhle } \\
\text { geärgert? } \\
\text { Waren Sie aufgrund von Problemen mit den Zähnen, dem Zahnersatz oder durch andere Probleme in der } \\
\text { Mundhöhle jemals ein wenig verlegen? }\end{array}$ \\
\hline 6 & $\begin{array}{l}\text { Soziale Beeinträchtigung } \\
\text { Haben Sie wegen Problemen mit den Zähnen, dem Zahnersatz oder anderer Probleme in der Mundhöhle, } \\
\text { darauf verzichtet, auszugehen? } \\
\text { Waren Sie aufgrund von Problemen mit den Zähnen, dem Zahnersatz oder wegen anderer Probleme in der } \\
\text { Mundhöhle weniger tolerant gegenüber Ihrem Partner oder Ihrer Familie? } \\
\text { Waren Sie wegen Problemen mit den Zähnen, dem Zahnersatz oder anderer Probleme in der Mundhöhle } \\
\text { leicht reizbar im Umgang mit anderen Menschen? }\end{array}$ \\
\hline 7 & $\begin{array}{l}\text { Behinderung bzw. starke Einschränkung im Alltag } \\
\text { Waren Sie aufgrund von Problemen mit den Zähnen, dem Zahnersatz oder durch andere Probleme in der } \\
\text { Mundhöhle nicht in der Lage die Gesellschaft anderer Menschen zu genießen? } \\
\text { Hatten Sie das Gefühl, dass aufgrund von Problemen mit den Zähnen, dem Zahnersatz oder anderen } \\
\text { Problemen in der Mundhöhle Ihr Leben insgesamt weniger befriedigend war? }\end{array}$ \\
\hline
\end{tabular}

Tabelle 2.6: OHIP-Subskaleneinteilung (Woelk 2007) 


\section{Resultate}

\subsection{Das Patientenkollektiv der Nachuntersuchung}

Von den anfangs 86 operierten Patienten konnten nach einer mittleren Beobachtungszeit von 52 Monaten noch 62 Patienten (38 Frauen und 26 Männer, Durschnittsalter 71 Jahre) nachuntersucht und befragt werden. Bei 24 Patienten war eine Nachuntersuchung nicht mehr möglich, da sie diese entweder ablehnten, fortgezogen oder verstorben waren. Die Drop-out-Rate betrug bei den Patienten mit Semadosimplantaten 26,5 \% und denen mit Xiveimplantaten 28,8\%. Durch den Chi-Quadrat-Test (p-Wert=0,81) konnte eine ungleichmäßige Verteilung der Drop-outs auf die beiden Gruppen ausgeschlossen werden.

Die Nachuntersuchung fand im Zeitraum von 2008-2009 in der Abteilung für zahnärztliche Chirurgie an der Universität Göttingen statt. In der Xivegruppe befanden sich 37 Patienten (20 Frauen und 17 Männer) mit einem durchschnittlichen Alter von 72 Jahren. In der Semadosgruppe waren 25 Patienten (20 Frauen und 5 Männer). Das Durchschnittsalter in dieser Gruppe lag bei 69 Jahren.

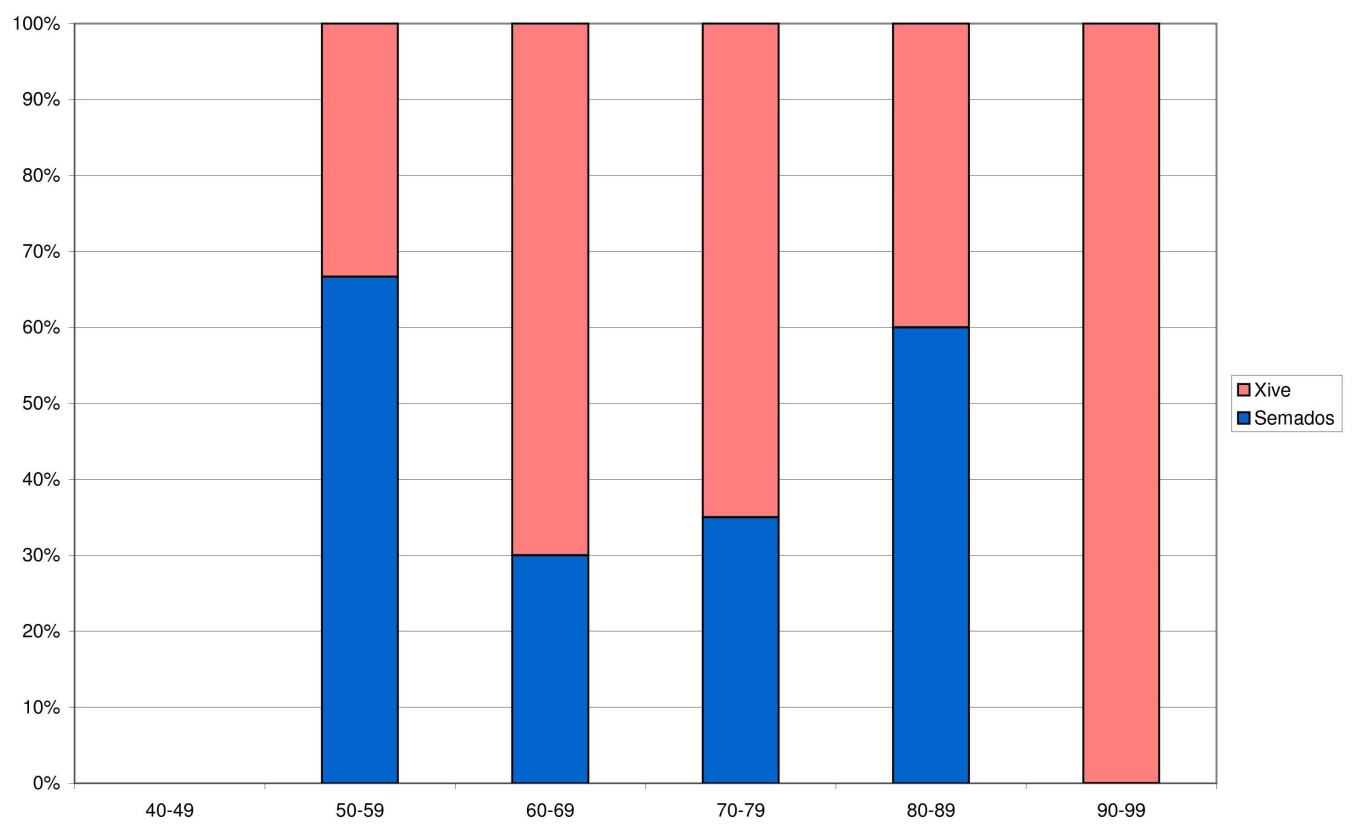

Abbildung 3.1: Altersverteilung der Patientengruppen 


\subsection{Lebensdaueranalyse}

Die Überlebenszeitanalyse für alle Implantatsysteme wird anhand des Kaplan-MeierSchätzer in Abbildung 3.2 dargestellt. Die getrennte Betrachtung der Überlebenszeit für das Xive- und Semadosimplantatsystem ist der Abbildung $3.3 \mathrm{zu}$ entnehmen. Die Gesamt-Überlebensrate in dieser Studie beträgt nach einem mittleren Beobachtungszeitraum von 52 Monaten 97,28\%. Dabei traten alle 5 Implantatverluste innerhalb der Xivegruppe nach 5, 6, 14,19 und 27 Monaten auf (siehe Abbildung 3.3 ). Daher ergibt sich für das Xiveimplantatsystems eine Überlebensrate von 95,19 \% und für das Semadosimplantatsystem von 100 \%. Beim Nachweis möglicher Einflussfaktoren auf die Überlebenszeit der Implantate stellte sich das Implantatsystem als signifikanter Einflussfaktor dar. Das Alter der Patienten und die Position der Implantate ergab keinen signifikanten Einfluss auf die Überlebenszeit der Implantate (Alter: $p=0,31 /$ Position: $\mathrm{p}=0,65)$.

Die Auswertung der Erfolgskriterien nach Jahn und d'Hoedt (1992) und Naert et al. (1992)/Snauwaert et al. (2000) unter Berücksichtigung der klinischen und radiologischen Parameter ergab eine Erfolgsquote von 95,11 \% und 97,28 \%. Die Ergebnisse der einzelnen Erfolgskriterien werden in Tabelle 3.2 dargestellt.

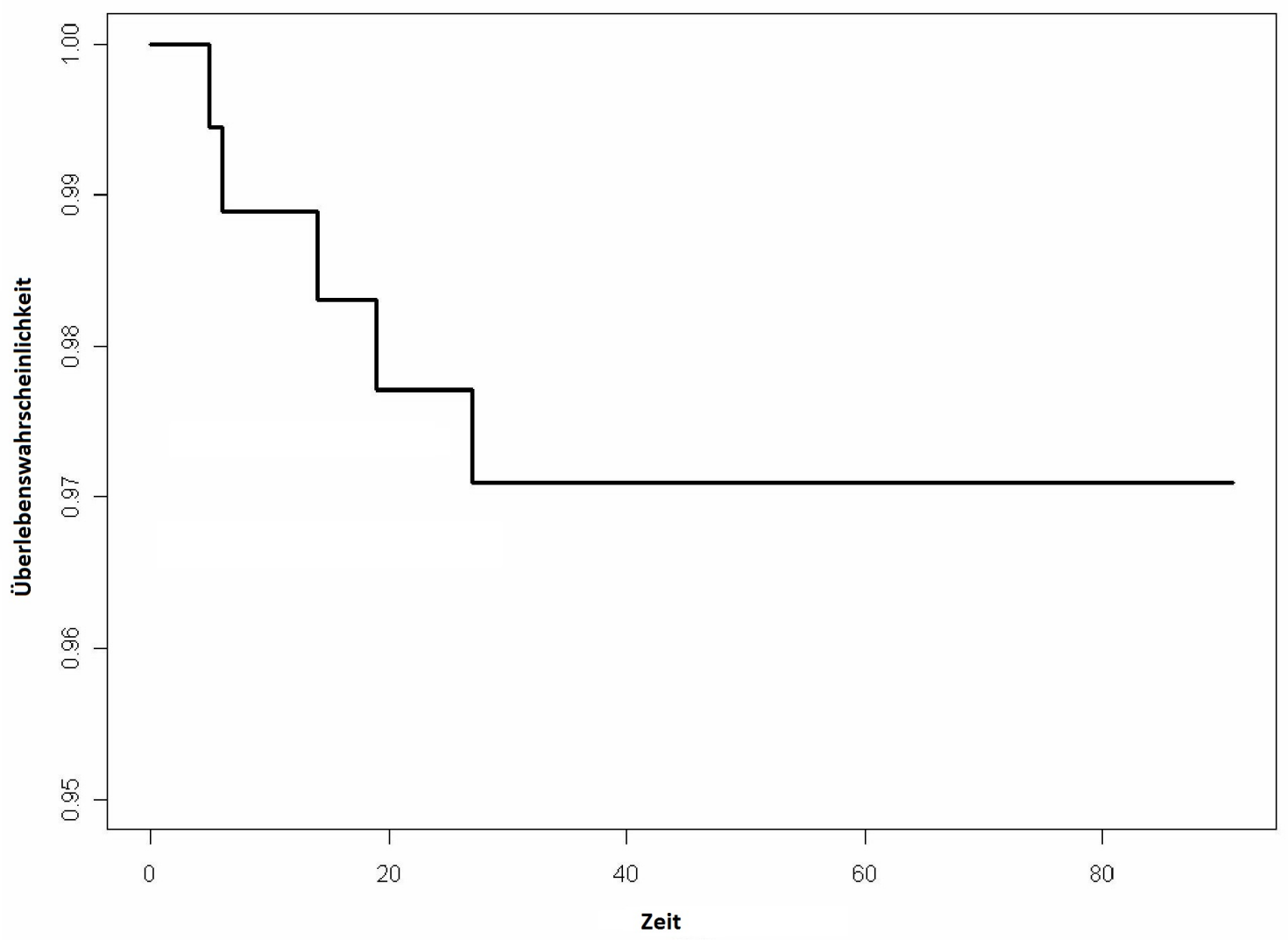

Abbildung 3.2: Kaplan-Meier-Kurve (gesamt) 


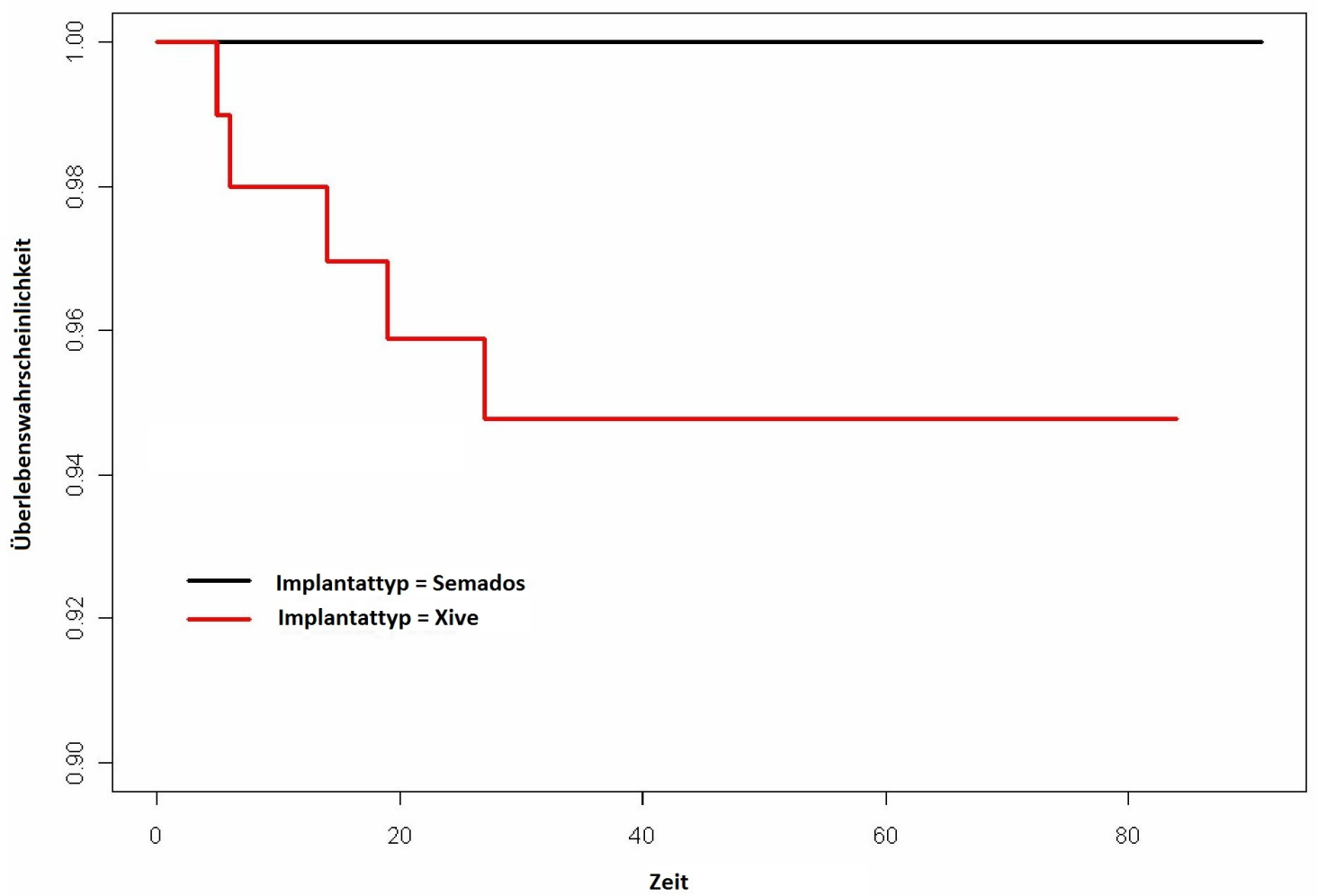

Abbildung 3.3: Kaplan-Meier-Kurve (Semados, Xive)

\begin{tabular}{|c|c|c|c|}
\hline $\begin{array}{l}\text { Jahn und d'Hoedt } \\
(1992)\end{array}$ & $\begin{array}{l}\text { n Misser- } \\
\text { folge }\end{array}$ & $\begin{array}{l}\text { Naert et al. (1992)/ } \\
\text { Snauwaert et al. (2000) }\end{array}$ & $\begin{array}{l}\text { n Misser- } \\
\text { folge }\end{array}$ \\
\hline Implantat in situ & 5 & Implantat in situ & 5 \\
\hline Periotestwert $<+8$ & 0 & Periotestwert $<+8$ & 0 \\
\hline Implantatspalt $<0,5 \mathrm{~mm}$ im OPG & 0 & $\begin{array}{l}\text { keine periimplantäre } \\
\text { Radiotranzluzenz }\end{array}$ & 0 \\
\hline $\begin{array}{l}\text { anguläre Knochendefekte }<3 / 10 \text { des } \\
\text { Implantats }\end{array}$ & 0 & keine Implantatfraktur & 0 \\
\hline $\begin{array}{l}\text { Sulkustiefe }<4 \mathrm{~mm} \text { bei zwei } \\
\text { Kontrollen }\end{array}$ & nicht bewertet & $\begin{array}{l}\text { Keine Schmerzen, Infektionen, } \\
\text { Parästhesien }\end{array}$ & 0 \\
\hline Patientenbewertung $<$ befriedigend & 4 & & \\
\hline Gesamte Misserfolge & 9 & Gesamte Misserfolge & 5 \\
\hline Erfolgsquote in \% & 95,11 & Erfolgsquote in $\%$ & 97,28 \\
\hline
\end{tabular}

Tabelle 3.2: Erfolgskriterien

\subsection{Ergebnisse des Periotests}

Im Rahmen der Nachuntersuchung wurden bei 62 Patienten die Periotestwerte bestimmt. Jedoch konnten insgesamt an nur 120 Implantaten die Periotestwerte gemes- 
sen werden, da es bei 4 Patienten zu einem Implantatverlust kam. Eine Übersicht über die erhobenen Messwerte wird in Tabelle 3.3 dargestellt. Des Weiteren wurden die Werte hinsichtlich möglicher Einflussfaktoren getestet. Als relevante Faktoren wurden die zwei verwendeten Implantatsysteme, das Alter der Patienten, die Verweildauer der Implantate in situ, sowie die Position im Kiefer (Regio 033 und 043) angenommen. Da es sich bei diesen Werten um eine Score handelt, wurde als Testverfahren eine nichtparametrische Kovarianzanalyse gewählt.

Keine der getesteten Kovariablen hatte einen signifikanten Einfluss auf die Periotestwerte. Lediglich die Verweildauer der Implantate kann mit einem p-Wert von 0.051 als auffällig beschrieben werden. Diese Tendenz, dass mit zunehmender Verweildauer der Implantate in der Mandibula, die Periotestwerte abgenommen haben und folglich die Stabilität der Implantate zugenommen hat, wird auch in Abbildung 3.6b ersichtlich.

\begin{tabular}{|c|c|c|c|c|c|c|c|c|}
\hline & $\mathrm{N}$ & MW & Median & Min. & Max. & Std.abw. & $\begin{array}{c}\text { Konfidenz } \\
-95 \%\end{array}$ & $\begin{array}{c}\text { Konfidenz } \\
95 \%\end{array}$ \\
\hline Xive & 70 & $-4,58$ & $-5,00$ & $-8,00$ & 3,00 & 1,80 & $-5,02$ & $-4,17$ \\
\hline Semados & 50 & $-5,27$ & $-5,00$ & $-8,00$ & $-2,00$ & 1,349 & $-5,66$ & $-4,88$ \\
\hline Gesamt & 120 & $-4,86$ & $-5,00$ & $-5,00$ & 3,00 & 1,64 & $-5,17$ & $-4,87$ \\
\hline
\end{tabular}

Tabelle 3.3: Übersicht der Periotestwerte

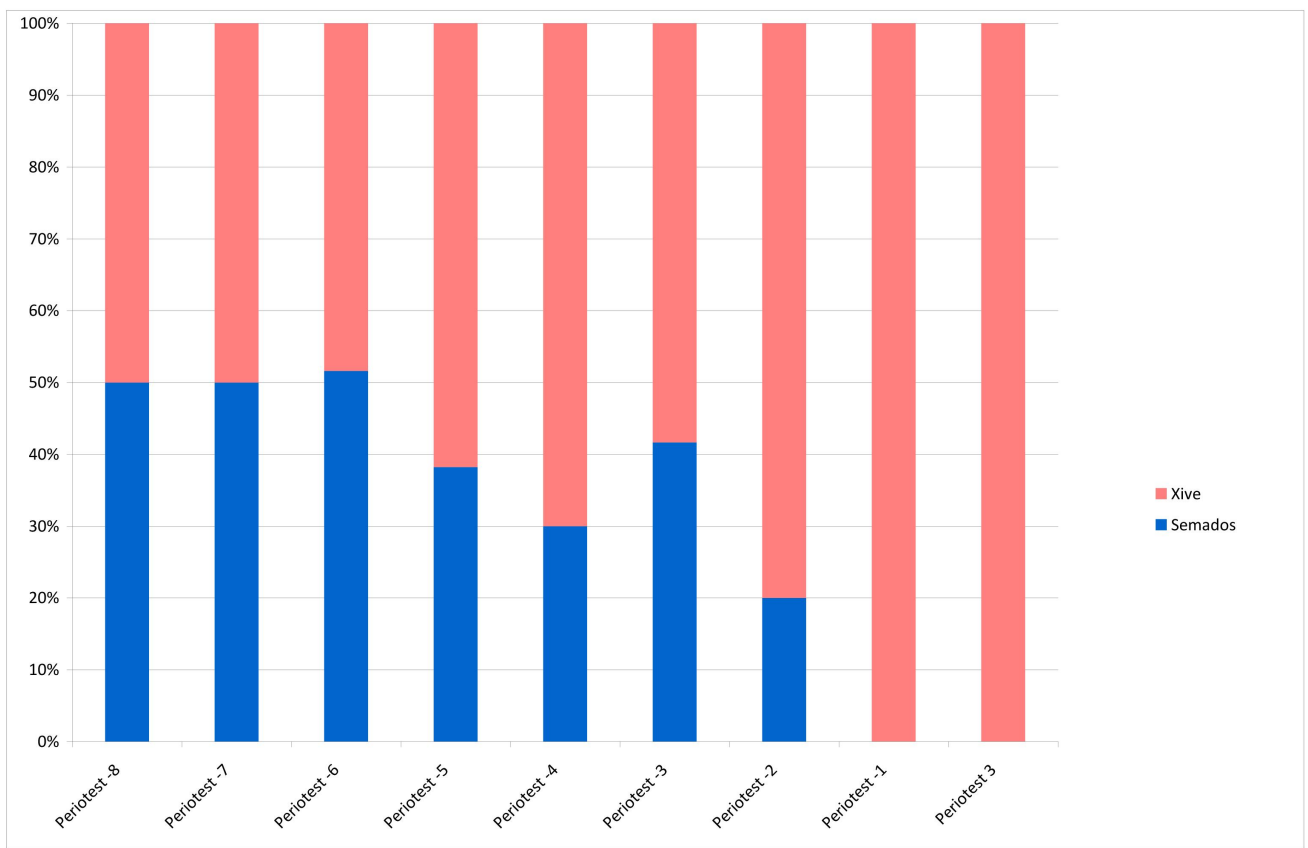

Abbildung 3.4: Verteilung der Periotestwerte (in Prozent) 


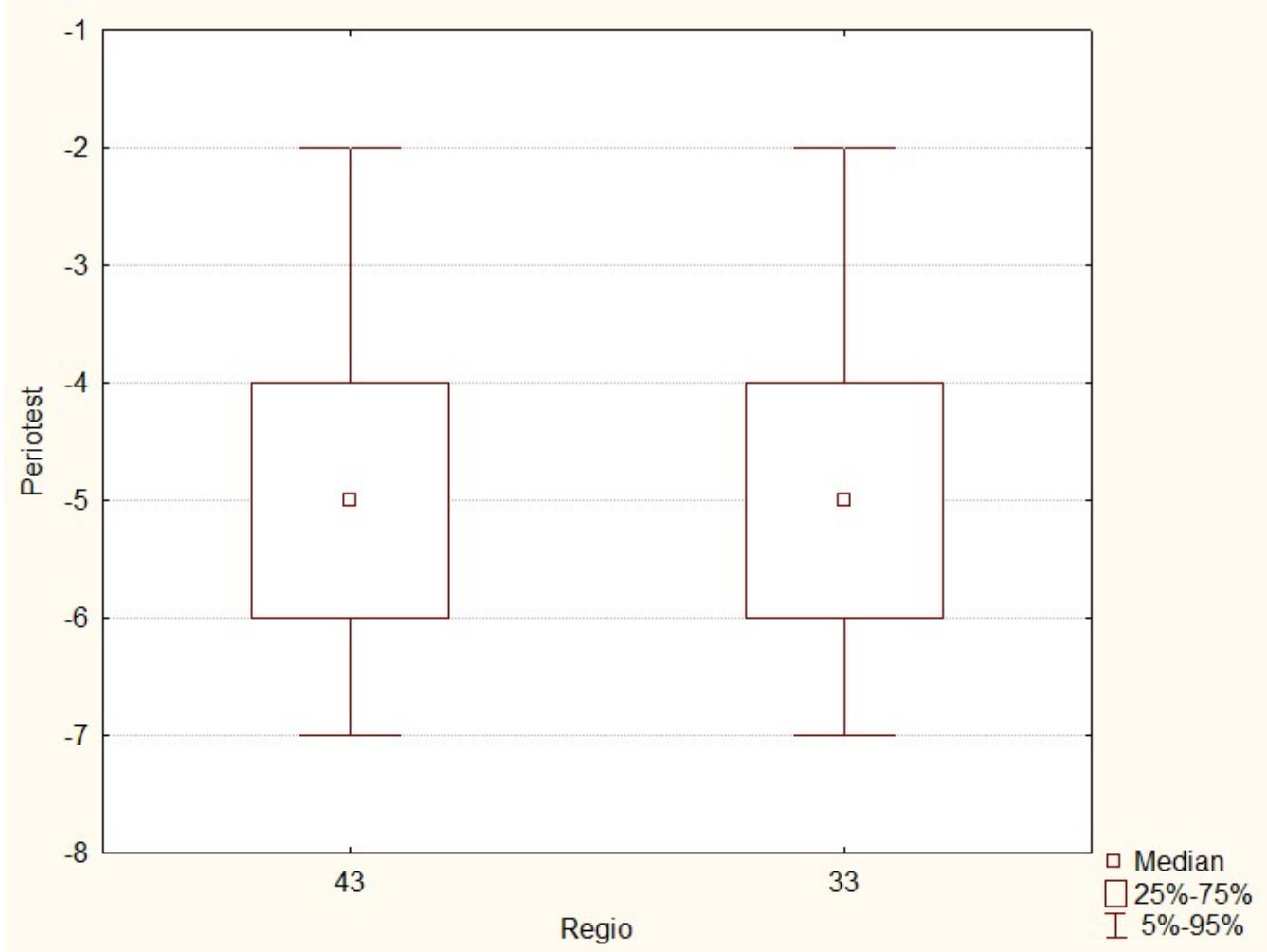

(a) Periotestwerte : Position im Kiefer

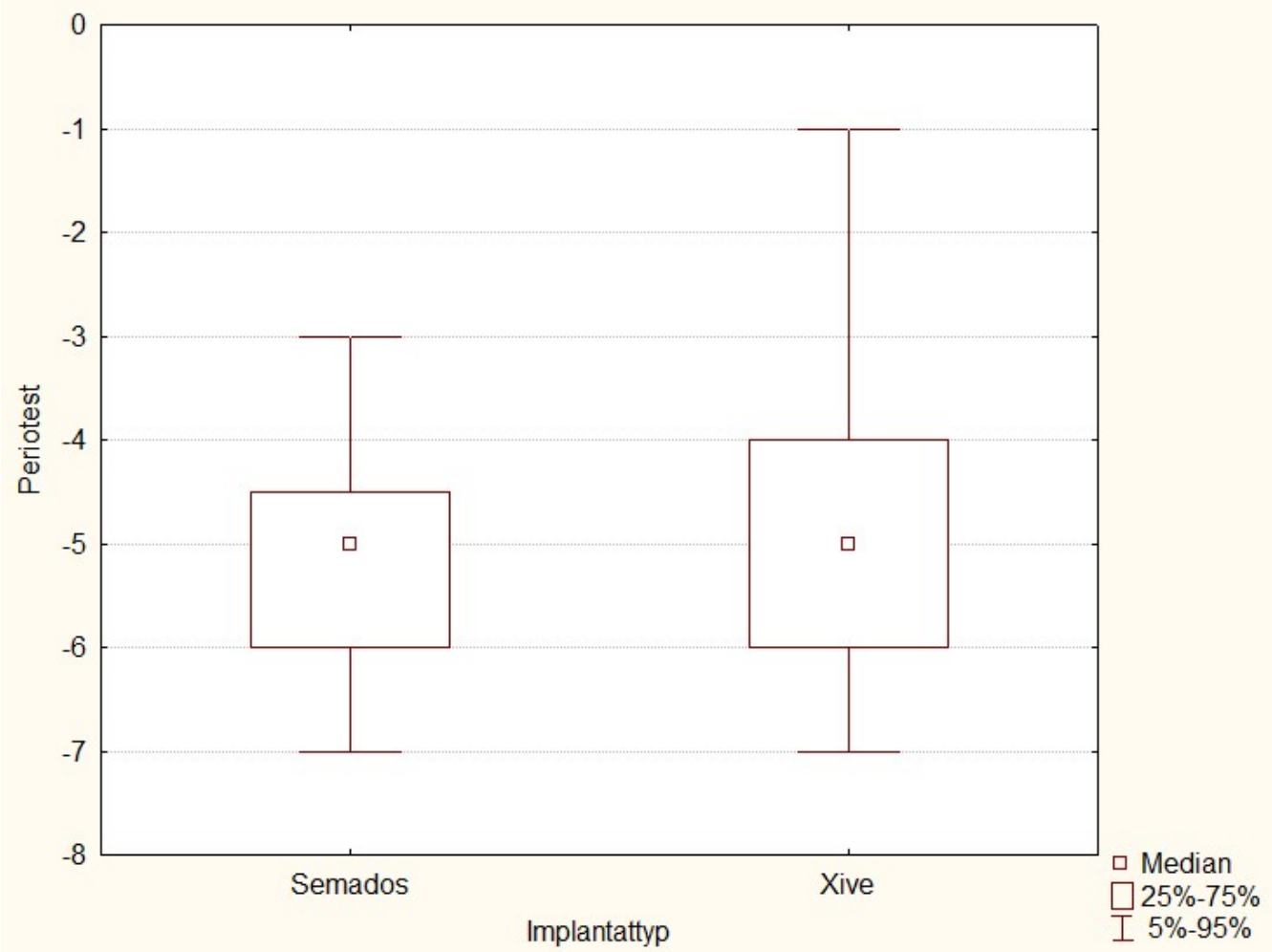

(b) Periotestwerte : Implantattyp

Abbildung 3.5: Boxplots der Periotestwerte 


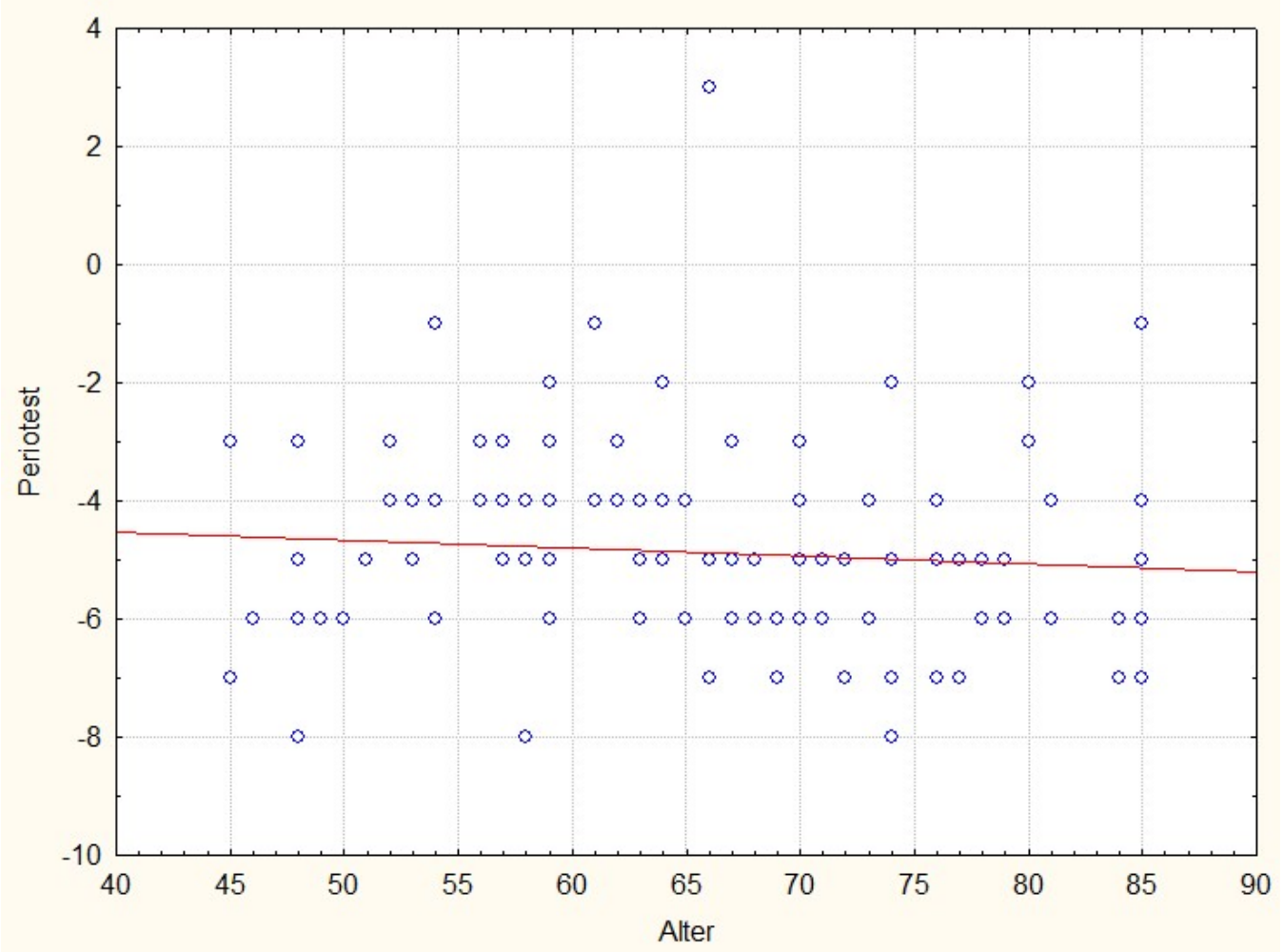

(a) Periotestwerte : Alter der Patienten (in Jahren)

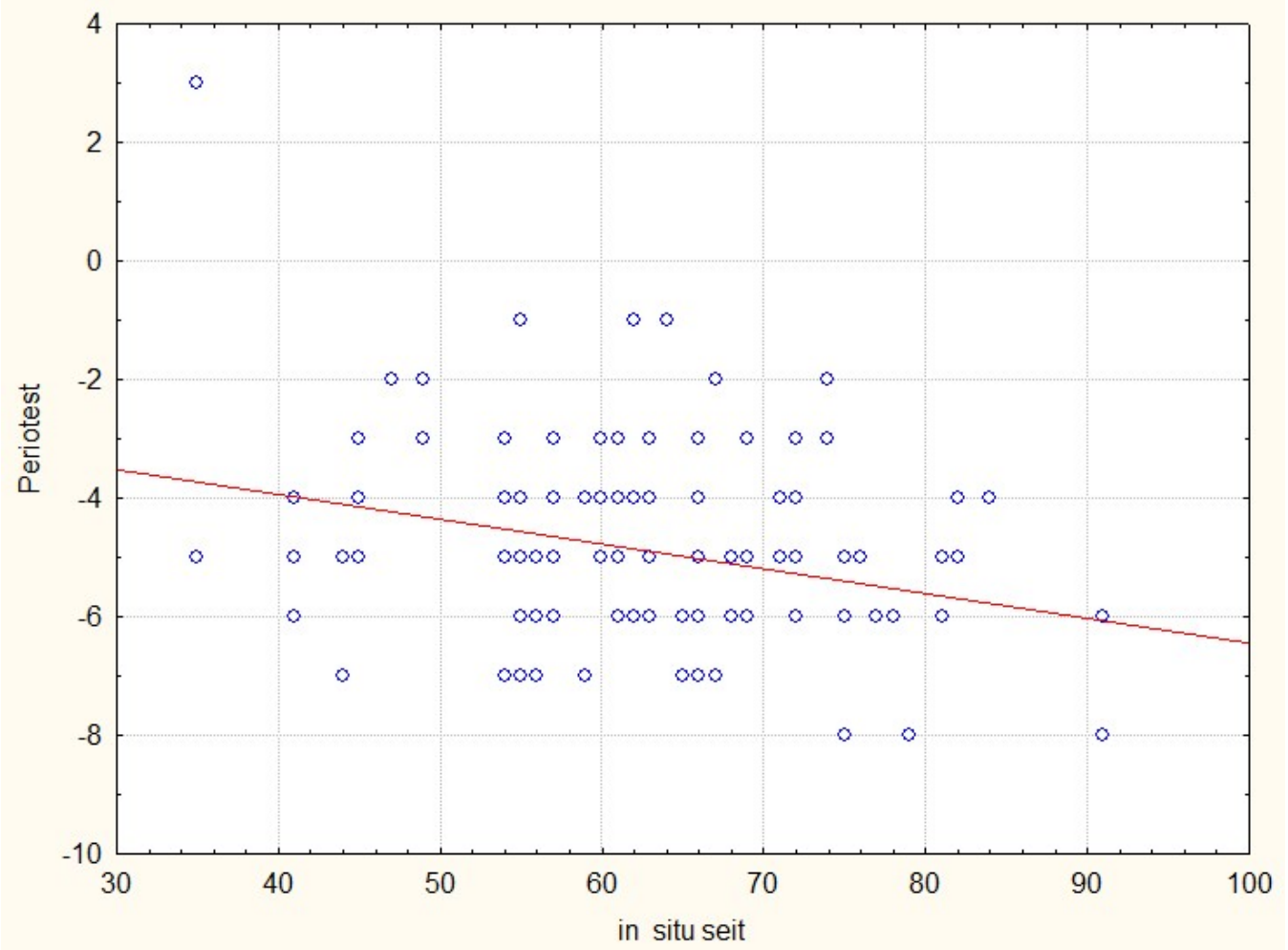

(b) Periotestwerte : Zeit in situ (in Monaten)

Abbildung 3.6: Scatterplots der Periotestwerte 


\begin{tabular}{lc}
\hline Einflussfaktoren & p-Wert \\
\hline Alter & 0,07 \\
Position im Kiefer & 0,62 \\
Verweildauer im Kiefer & 0,05 \\
Implantatsystem & 0,23 \\
\hline
\end{tabular}

Tabelle 3.4: Ergebnisse der Kovarianzanalyse für die Periotestwerte

\subsection{Ergebnisse des horizontalen Knochenabbaus}

Die Tabelle 3.5 veranschaulicht die deskriptive Datenanalyse des horizontalen Knochenabbaus. Dabei wurde hinsichtlich des Implantattyps und der Implantatposition differenziert. Die Mittelwerte des mesialen Knochenabbaus betrugen am Implantat 033 1,13 $\mathrm{mm}$ und an 043 1,12 mm. Distal wurden an 033 ein Mittelwert von 0,96 $\mathrm{mm}$ und an 043 von $0,95 \mathrm{~mm}$ festgestellt.

\begin{tabular}{|c|c|c|c|c|c|c|c|c|c|}
\hline & & $\mathrm{N}$ & MW & Median & Min. & Max. & Std.abw. & $\begin{array}{c}\text { Konfidenz } \\
-95 \%\end{array}$ & $\begin{array}{c}\text { Konfidenz } \\
95 \%\end{array}$ \\
\hline \multirow[t]{3}{*}{33 mesial } & Xive & 49 & 1,19 & 0,90 & 0 & 5,90 & 1,10 & 0,87 & 1,51 \\
\hline & Semados & 34 & 1,04 & 0,85 & 0 & 4,50 & 0,85 & 0,74 & 1,34 \\
\hline & Gesamt & 83 & 1,13 & 0,90 & 0 & 5,90 & 1,00 & 0,91 & 1,35 \\
\hline \multirow[t]{3}{*}{33 distal } & Xive & 49 & 1,09 & 0,80 & 0 & 4,80 & 0,98 & 0,81 & 1,37 \\
\hline & Semados & 34 & 0,76 & 0,70 & 0 & 2,00 & 0,48 & 0,59 & 0,93 \\
\hline & Gesamt & 83 & 0,96 & 0,70 & 0 & 4,80 & 0,82 & 0,78 & 1,14 \\
\hline \multirow[t]{3}{*}{43 mesial } & Xive & 50 & 1,18 & 0,95 & 0 & 4,50 & 0,86 & 0,93 & 1,42 \\
\hline & Semados & 34 & 1,04 & 0,95 & 0 & 2,40 & 0,60 & 0,83 & 1,25 \\
\hline & Gesamt & 84 & 1,12 & 0,95 & 0 & 4,50 & 0,77 & 0,95 & 1,29 \\
\hline \multirow[t]{3}{*}{43 distal } & Xive & 50 & 1,09 & 0,90 & 0 & 3,00 & 0,81 & 0,86 & 1,32 \\
\hline & Semados & 34 & 0,95 & 0,80 & 0 & 2,30 & 0,64 & 0,72 & 1,17 \\
\hline & Gesamt & 84 & 1,03 & 0,90 & 0 & 3,00 & 0,74 & 0,87 & 1,19 \\
\hline
\end{tabular}

Tabelle 3.5: Messdaten des horizontalen Knochenabbaus

Zur Datenanalyse des horizontalen Knochenabbaus wurde eine Kovarianzanalyse (ANCOVA) durchgeführt. Es wurden, wie auch schon zuvor bei der Analyse der Periotestwerte beschrieben, die Kovariablen (Implantattyp, Alter der Patienten, Zeit des Implantats in situ und Position im Kiefer) getestet. In diesem Zusammenhang konnte ein signifikanter Einfluss des Implantatsystems und der Verweildauer der Implantate im 
Kiefer auf den distalen Knochenabbau festgestellt werden (siehe Tabelle 3.6). Folglich zeigte sich beim Xive - Implantat distal ein deutlich größerer Knochenabbau als beim Semados-Implantat. Darüber hinaus fiel auf, dass der horizontale Knochenabbau, unabhängig vom Implantatsystem und der Position des Implantats, mesial im Mittel um 0,14 mm größer war als distal (siehe Abbildung 3.5 und 3.7). Bei allen anderen Kovariablen konnte kein signifikanter Einfluss auf den horizontalen Knochenabbau festgestellt werden.

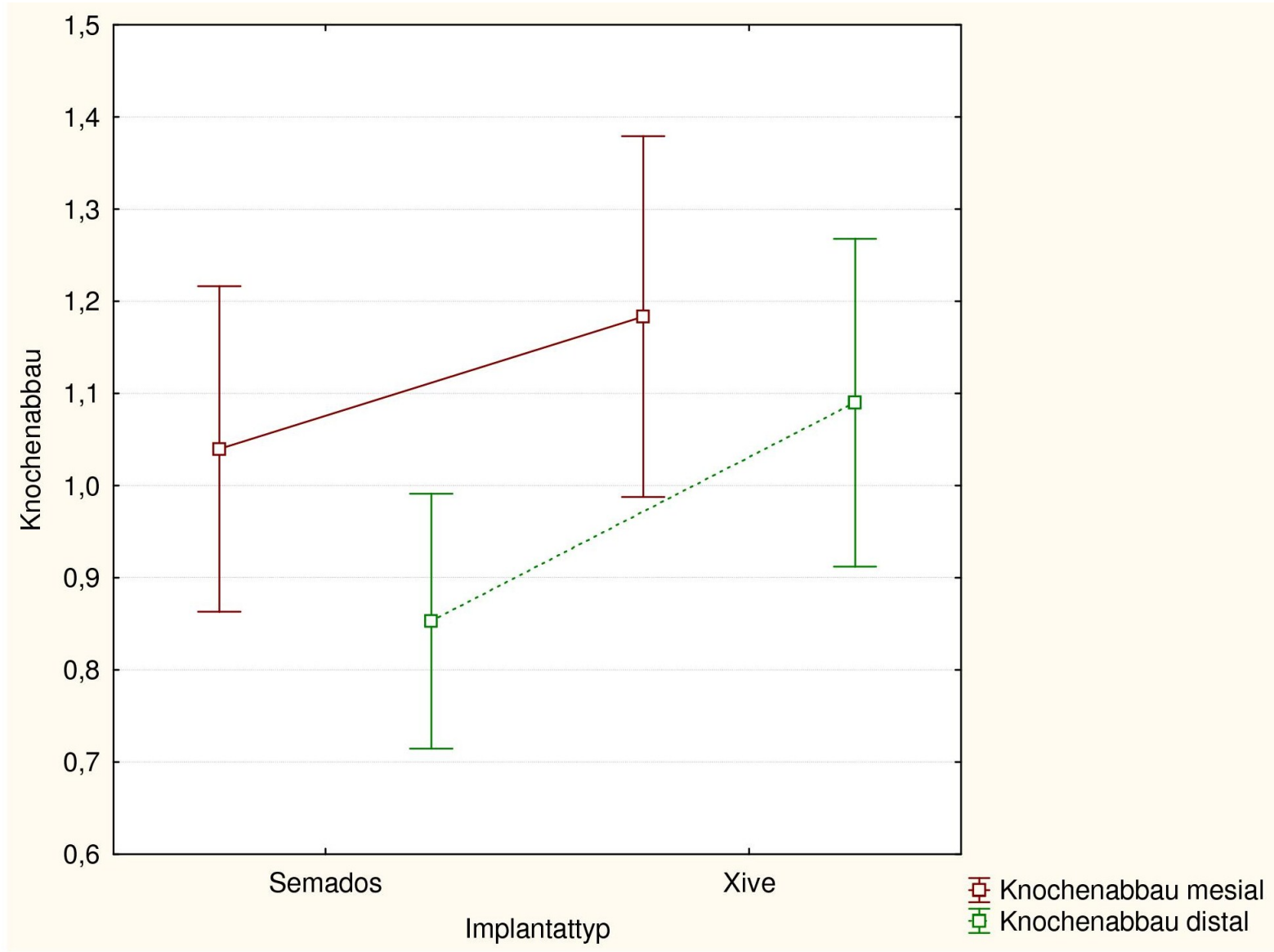

Abbildung 3.7: Knochenabbau (in mm) : Implantattyp 


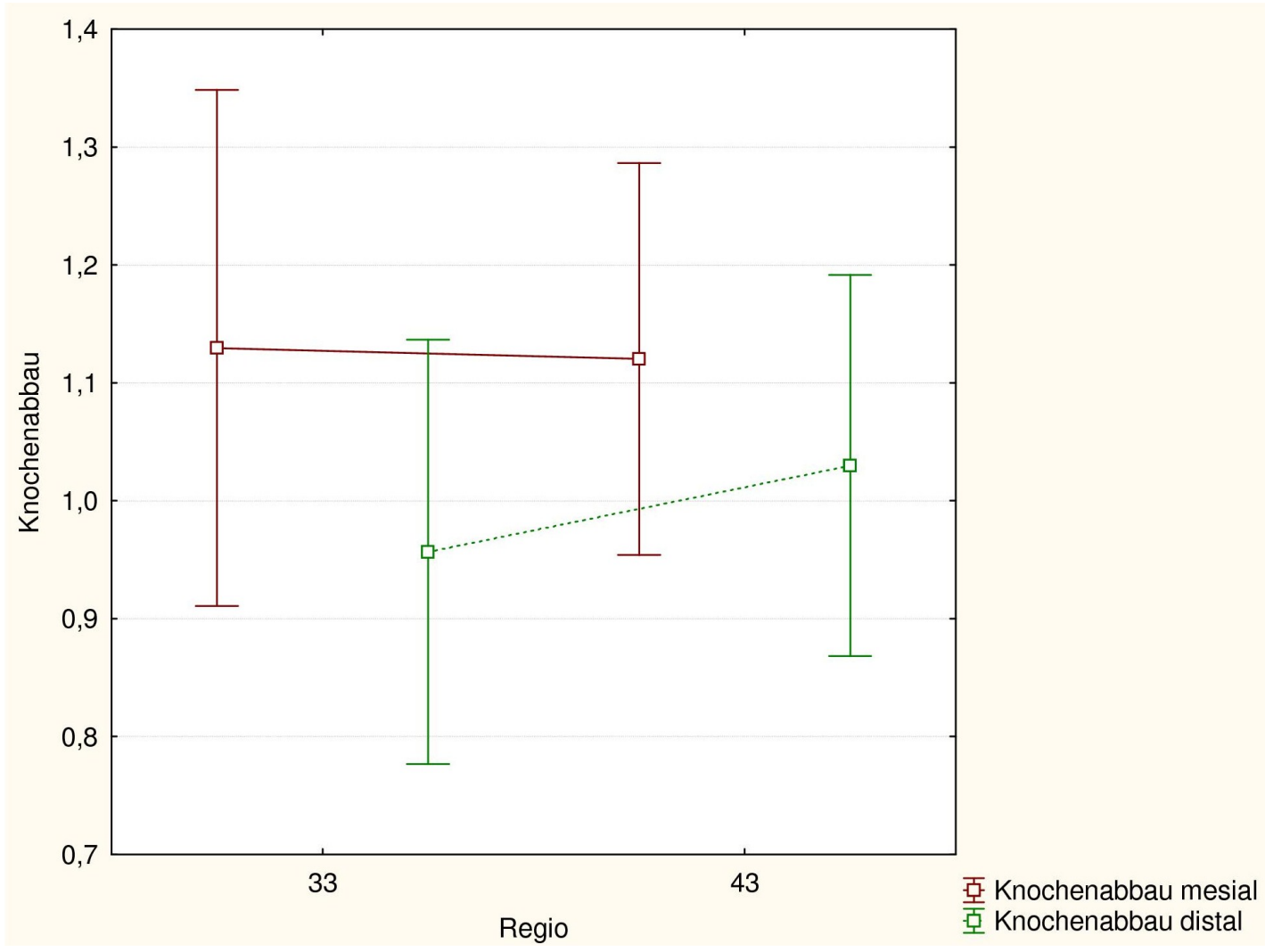

Abbildung 3.8: Knochenabbau (in mm) : Implantatposition

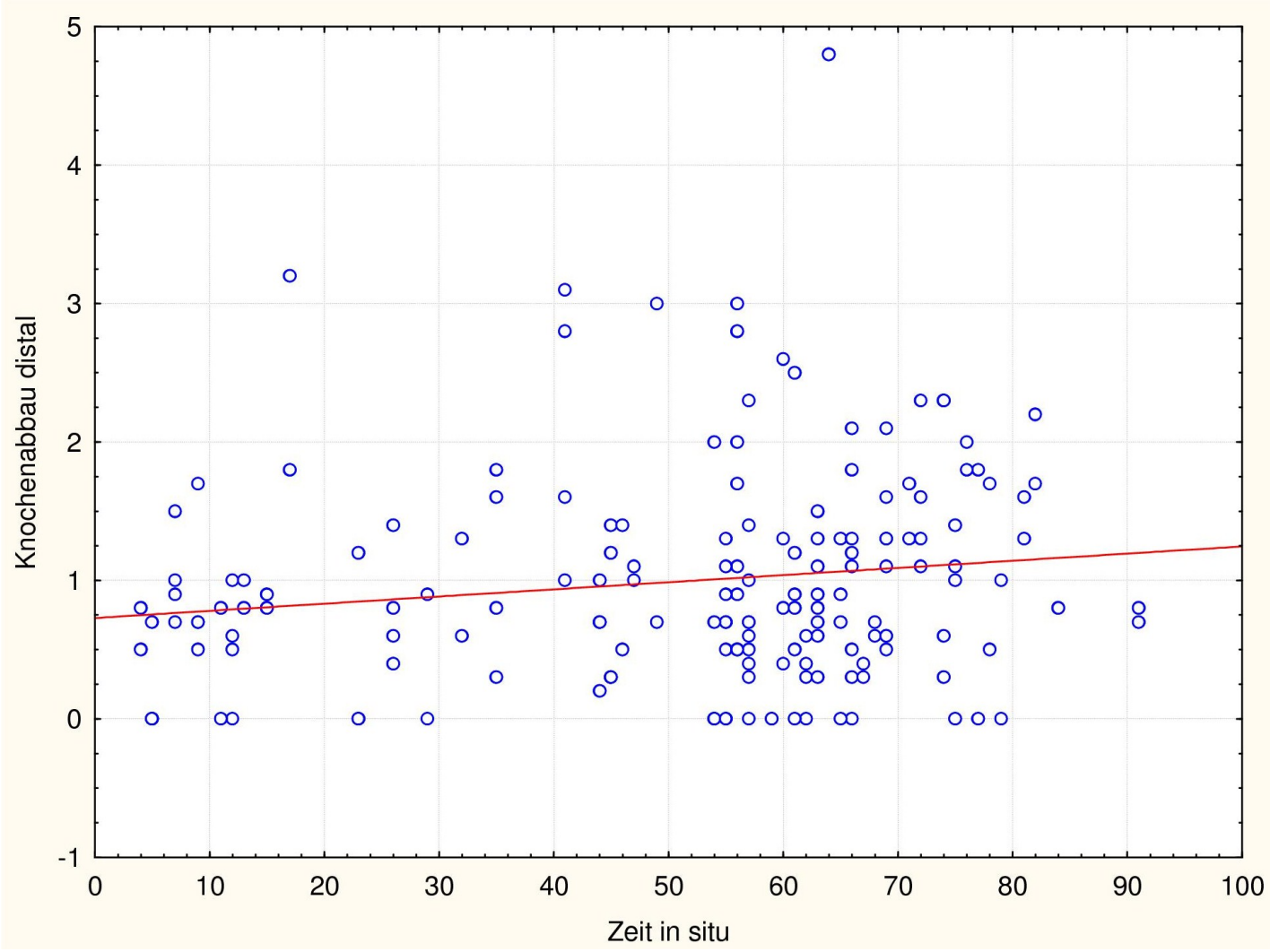

Abbildung 3.9: Knochenabbau (distal) : Zeit in situ (Monate) 


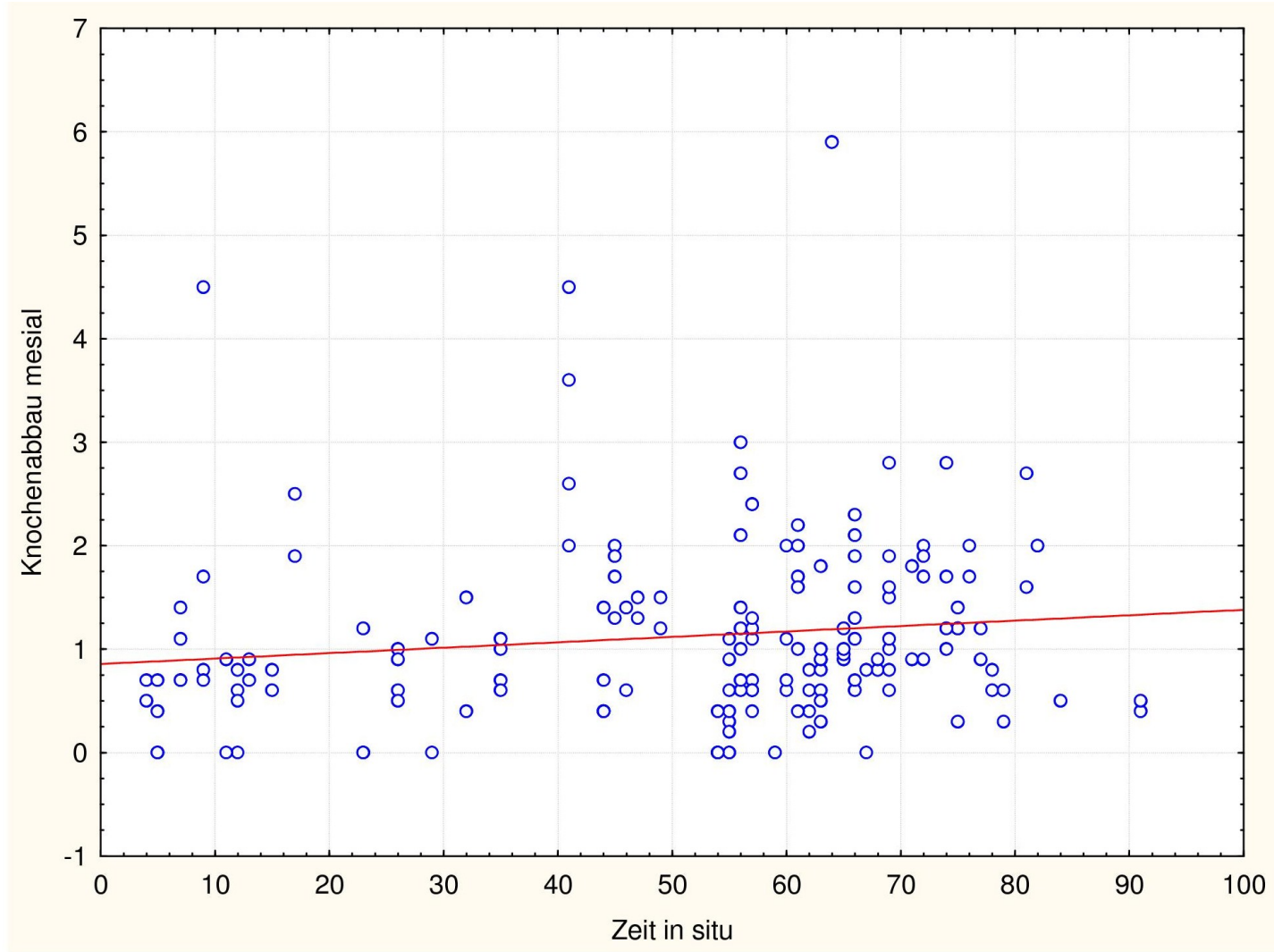

Abbildung 3.10: Knochenabbau (mesial) : Zeit in situ (Monate)

\begin{tabular}{lcc}
\hline Einflussfaktoren & p-Wert mesial & p-Wert distal \\
\hline Alter & 0,09 & 0,37 \\
Position im Kiefer & 0,96 & 0,54 \\
Verweildauer im Kiefer & 0,06 & 0,03 \\
Implantatsystem & 0,18 & 0,03 \\
\hline
\end{tabular}

Tabelle 3.6: Ergebnisse der Kovarianzanalyse für den horizontalen Knochenabbau

\subsection{Ergebnisse der Sondierungstiefen}

In Tabelle 3.7 werden die deskriptiven Daten der periimplantären Sondierungstiefen dargestellt. Die Mittelwerte der mesialen periimplantären Sondierungstiefen betrugen am Implantat 033 2,59 $\mathrm{mm}$ und an 043 2,37 $\mathrm{mm}$. Distal betrugen die Mittelwerte an 033 2,45 mm und an 043 2,42 $\mathrm{mm}$. Bei der Kovarianzanalyse konnte ein signifikanter Einfluss der Verweildauer der Implantate im Kiefer auf die mesiale periimplantäre Sondierungstiefe beobachtet werden (siehe Tabelle 3.6). Mit zunehmender Zeit der Implantate in situ nahmen die Sondierungstiefen ab. Alle anderen Kovariablen hatten keinen signifikanten Einfluss. 


\begin{tabular}{|c|c|c|c|c|c|c|c|c|c|}
\hline & & $\mathrm{N}$ & MW & Median & Min. & Max. & Std.abw. & $\begin{array}{c}\text { Konfidenz } \\
-95 \%\end{array}$ & $\begin{array}{c}\text { Konfidenz } \\
95 \%\end{array}$ \\
\hline \multirow[t]{3}{*}{33 mesial } & Xive & 35 & 2,65 & 3,00 & 2,00 & 4,00 & 0,68 & 2,41 & 2,87 \\
\hline & Semados & 25 & 2,50 & 2,75 & 2,00 & 3,00 & 0,50 & 2,31 & 2,73 \\
\hline & Gesamt & 60 & 2,59 & 3,00 & 2,00 & 4,00 & 0,61 & 2,43 & 2,75 \\
\hline \multirow[t]{3}{*}{33 distal } & Xive & 35 & 2,42 & 2,00 & 1,00 & 4,00 & 0,60 & 2,21 & 2,62 \\
\hline & Semados & 25 & 2,48 & 2,50 & 1,00 & 4,00 & 0,66 & 2,22 & 2,78 \\
\hline & Gesamt & 60 & 2,45 & 2,00 & 1,00 & 4,00 & 0,62 & 2,45 & 2,29 \\
\hline \multirow[t]{3}{*}{43 mesial } & Xive & 35 & 2,40 & 2,00 & 1,00 & 4,00 & 0,69 & 2,16 & 2,62 \\
\hline & Semados & 25 & 2,34 & 2,00 & 1,00 & 4,00 & 0,63 & 2,09 & 2,62 \\
\hline & Gesamt & 60 & 2,37 & 2,00 & 1,00 & 4,00 & 0,66 & 2,38 & 2,20 \\
\hline \multirow[t]{3}{*}{43 distal } & Xive & 35 & 2,37 & 2,00 & 2,00 & 4,00 & 0,59 & 2,16 & 2,56 \\
\hline & Semados & 25 & 2,50 & 2,00 & 2,00 & 4,00 & 0,65 & 2,25 & 2,80 \\
\hline & Gesamt & 60 & 2,42 & 2,00 & 2,00 & 4,00 & 0,62 & 2,27 & 2,58 \\
\hline
\end{tabular}

Tabelle 3.7: Sondierungstiefen

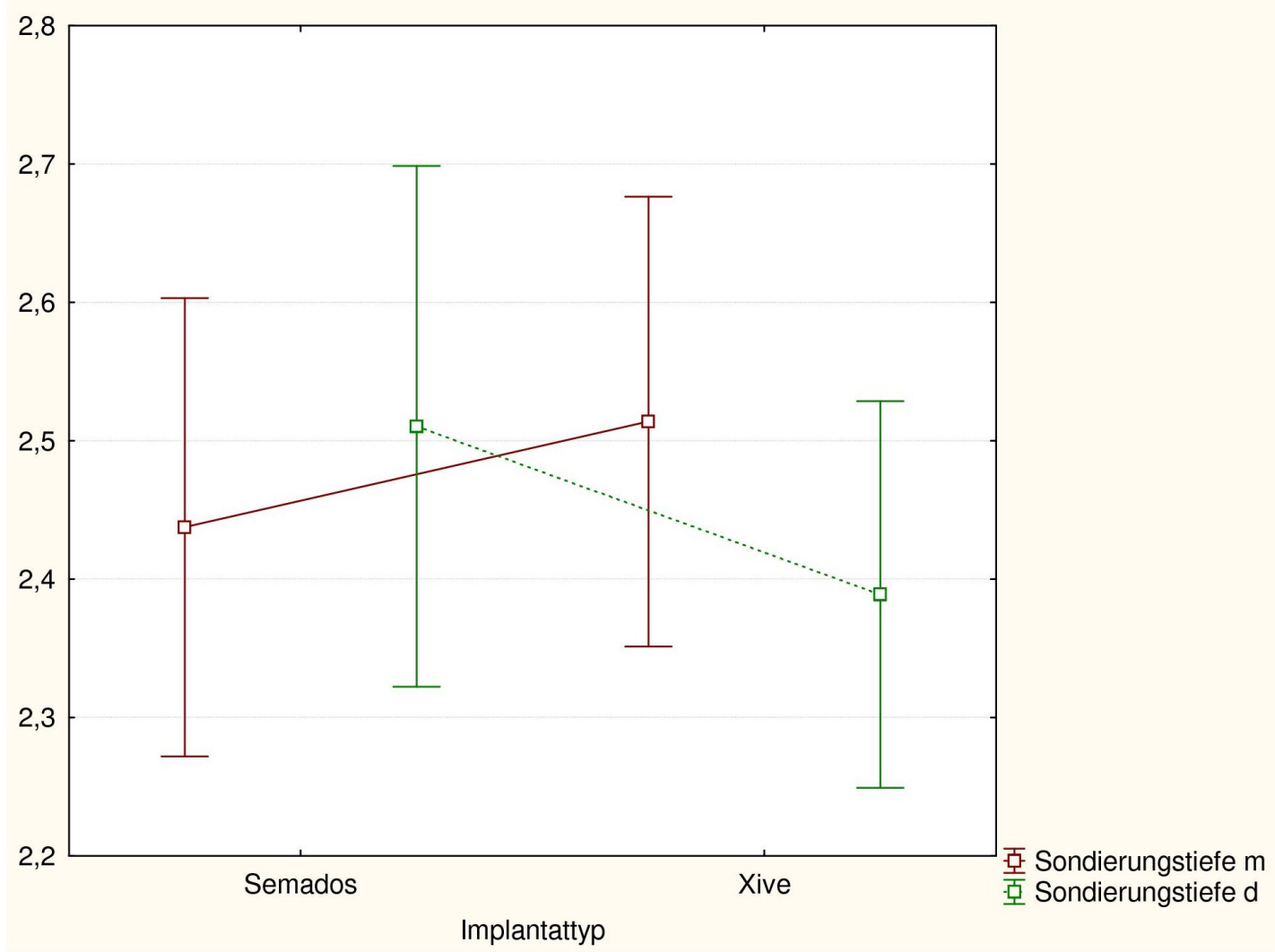

Abbildung 3.11: Mittelwert/Fehlerplot für Sondierungstiefen:Implantattyp 


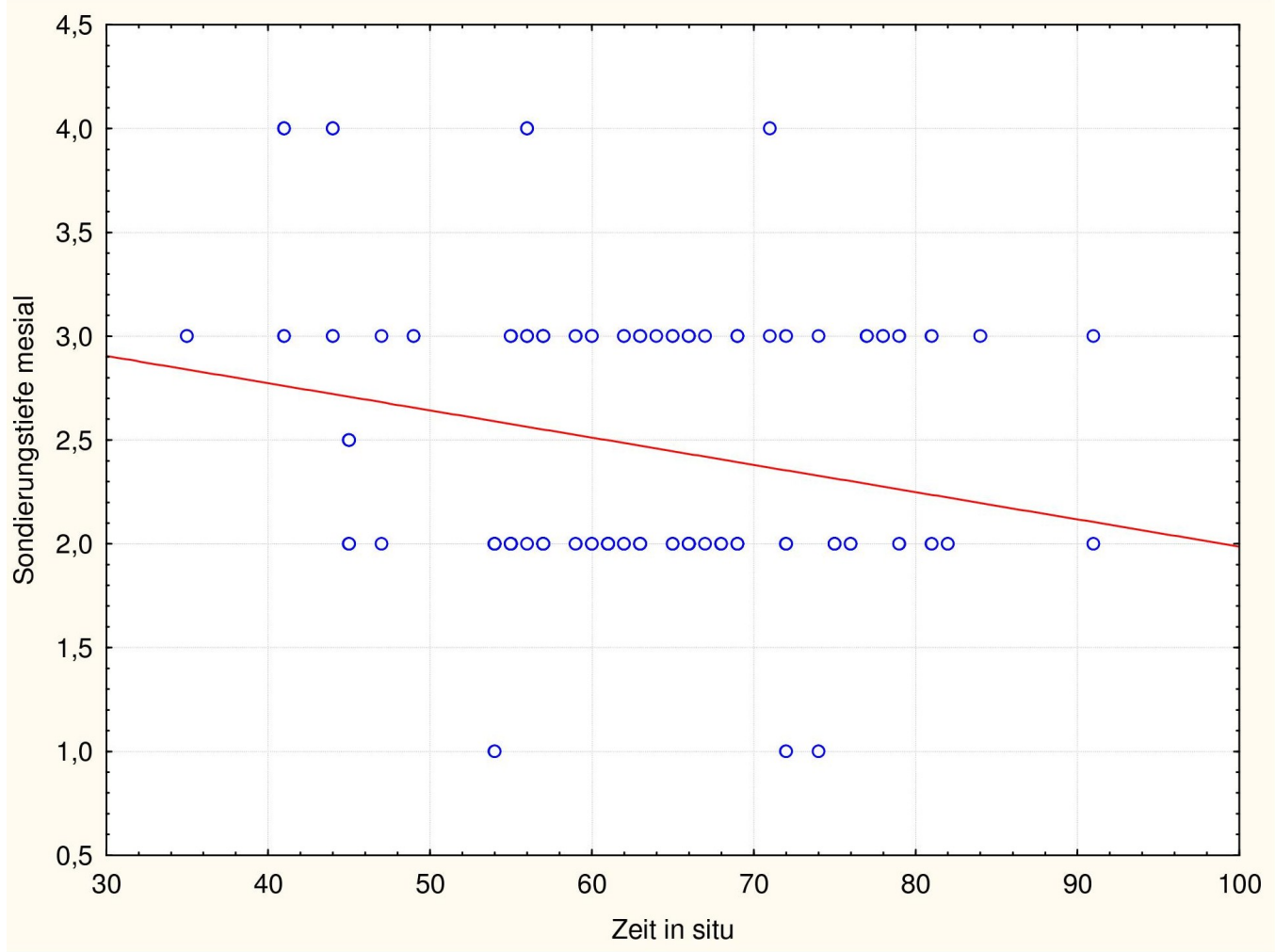

Abbildung 3.12: Sondierungstiefen mesial (in $\mathrm{mm}$ ) : Zeit in situ

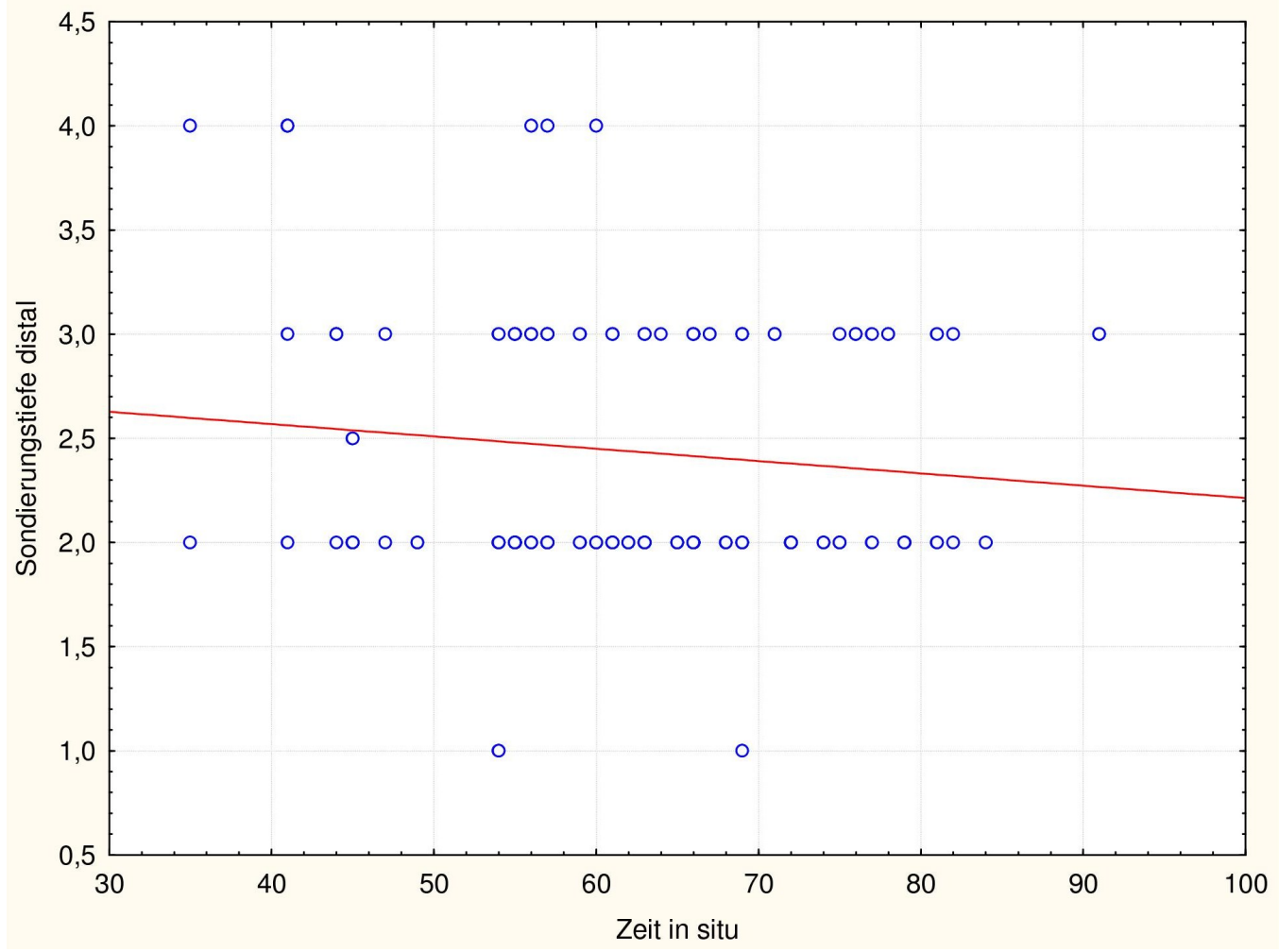

Abbildung 3.13: Sondierungstiefen distal (in $\mathrm{mm}$ ) : Zeit in situ 


\begin{tabular}{lcc}
\hline Einflussfaktoren & p-Wert mesial & p-Wert distal \\
\hline Alter & 0,67 & 0,05 \\
Position im Kiefer & 0,06 & 0,86 \\
Verweildauer im Kiefer & 0,01 & 0,09 \\
Implantatsystem & 0,85 & 0,25 \\
\hline
\end{tabular}

Tabelle 3.8: Ergebnisse der Kovarianzanalyse für die Sondierungstiefen

\subsection{Blutung auf Sondierung}

Es kam an sechs Implantaten zu Blutungen auf Sondierung. Alle Blutungen traten dabei an Xive- Implantaten auf. Die nichtparametrische Kovarianzanalyse bestätigte diesen signifikanten Unterschied. Alle anderen Kovariablen hatten keinen signifikanten Einfluss (siehe Tabelle 3.9) .

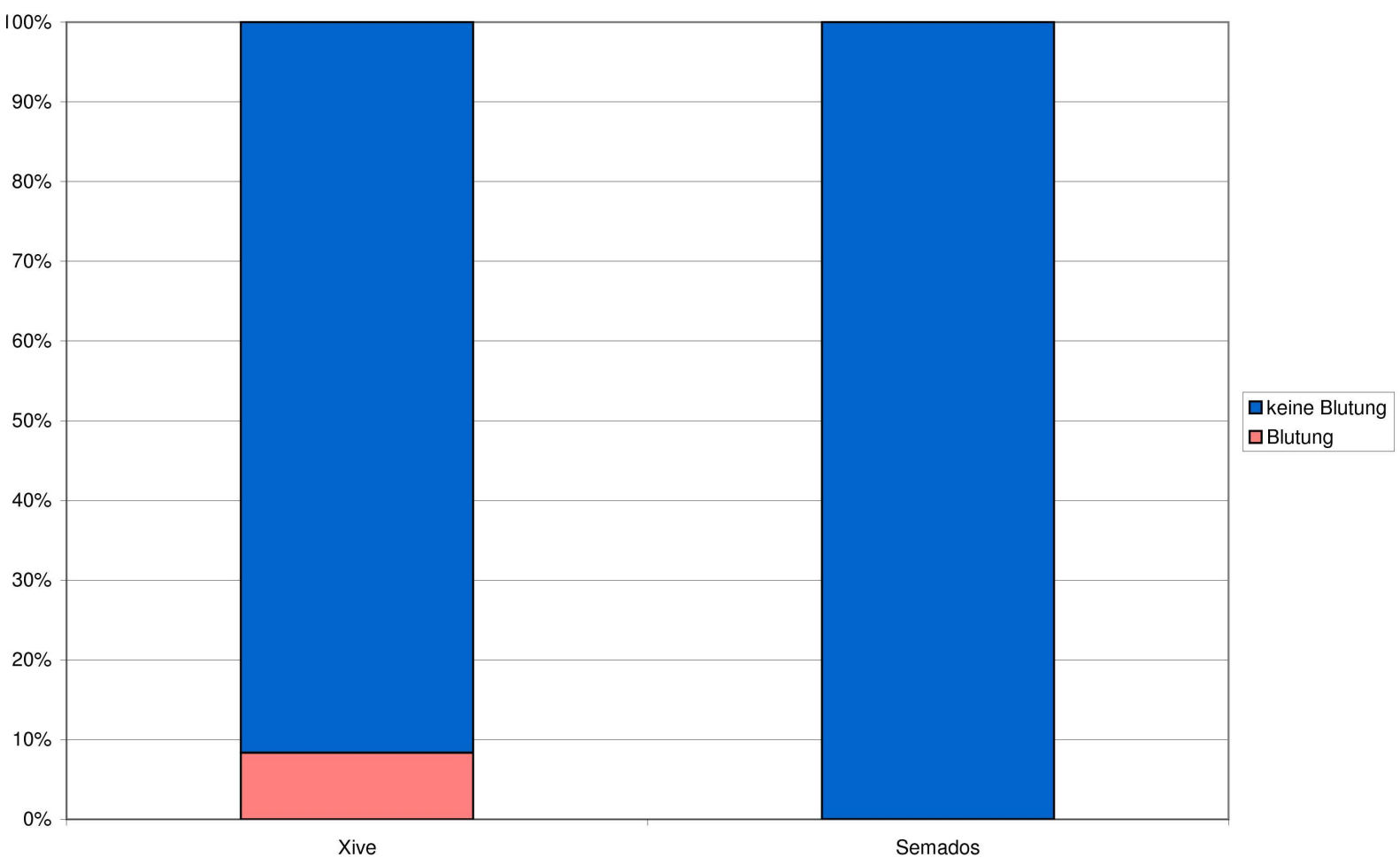

Abbildung 3.14: Anzahl der Blutungen 


\begin{tabular}{lc}
\hline Einflussfaktoren & p-Wert \\
\hline Alter & 0,66 \\
Position im Kiefer & 1 \\
Verweildauer im Kiefer & 0,72 \\
Implantatsystem & 0,008 \\
\hline
\end{tabular}

Tabelle 3.9: Ergebnisse der Kovarianzanalyse für Sondierungsblutungen

\subsection{Fragebogen zur Patientenzufriedenheit}

\subsubsection{Gesamtresultat der Behandlung}

Die Bewertung des Gesamtresultats der Behandlung wurde anhand von 62 Patientenfragebögen ausgewertet. Dabei beurteilten $48 \%$ der befragten Patienten das Behandlungsergebnis auf der Skala des deutschen Schulnotensystems mit „sehr gut“, 39 \% mit „gut", $6 \%$ mit „befriedigend“, $5 \%$ mit ,ausreichend“ und $2 \%$ mit „mangelhaft“. Um außerdem mögliche signifikante Unterschiede hinsichtlich der beiden verwendeten Implantatsysteme ermitteln zu können, wurde ein Man-Whitney-U -Test durchgeführt. Es wurde ein $\mathrm{p}>0,05$ ermittelt, so dass keine signifikanten Unterschiede in der Bewertung des Gesamtresultats bezüglich der Implantatsysteme festgestellt werden konnten.

\begin{tabular}{lcccccccc}
\hline & N & MW & Median & Min. & Max. & Std.abw. & $\begin{array}{c}\text { Konfidenz } \\
-95 \%\end{array}$ & $\begin{array}{c}\text { Konfidenz } \\
95 \%\end{array}$ \\
\hline Xive & 37 & 1,78 & 2,00 & 1,00 & 5,00 & 0,92 & 1,78 & 1,48 \\
Semados & 25 & 1,68 & 1,00 & 1,00 & 4,00 & 0,90 & 1,31 & 2,05 \\
\hline Gesamt & 62 & 1,74 & 2,00 & 1,00 & 5,00 & 0,90 & 1,51 & 1,97 \\
\hline
\end{tabular}

Abbildung 3.15: Patientenbewertung des Gesamtresultats 


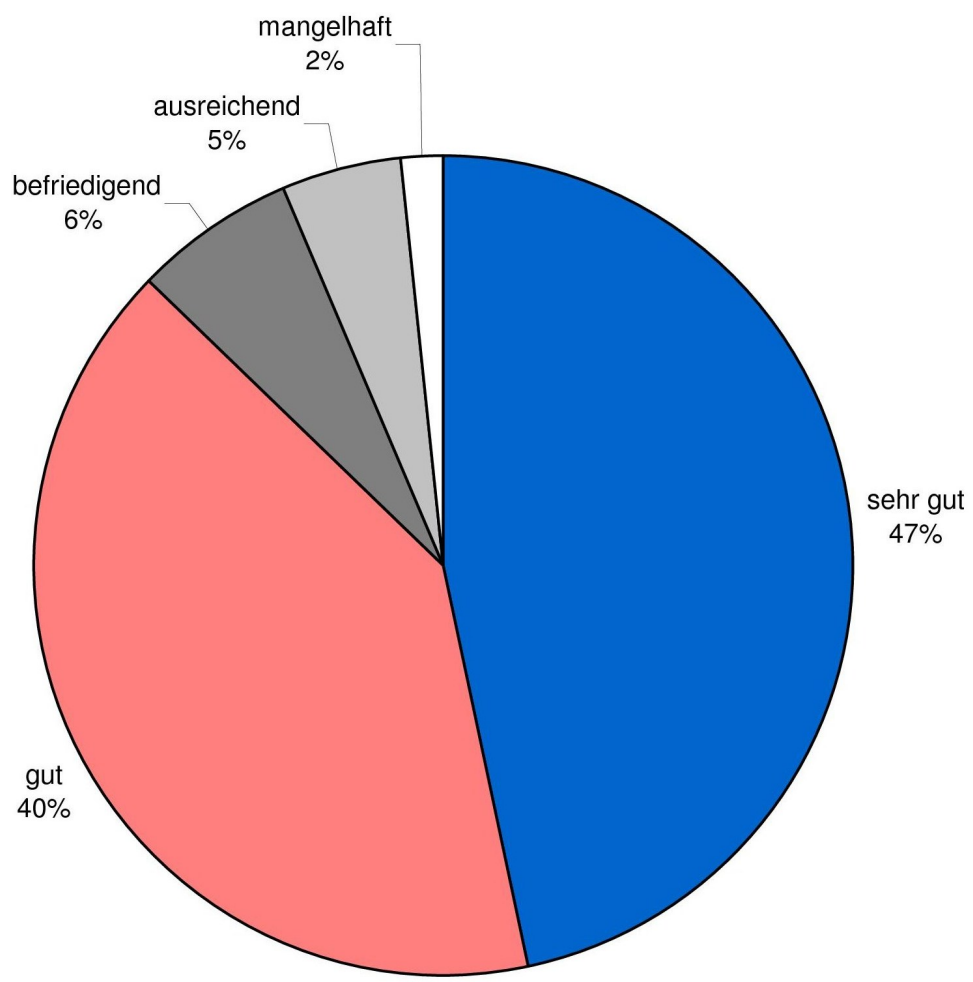

Abbildung 3.16: Gesamtresultat der Behandlung

\subsubsection{Auswertung des Halts der Prothese mit Kugelattachments}

Den Halt der Prothese beurteilten die 62 befragten Patienten mit einer Durchschnittsnote von 2,35. Beim Vergleich der beiden Implantatsysteme Semados und Xive mittels Mann-Whitney-U-Test konnte keine statistische Signifikanz festgestellt werden.

\begin{tabular}{lcccccccc}
\hline & N & MW & Median & Min. & Max. & Std.abw. & Konfidenz & \multicolumn{2}{c}{ Konfidenz } \\
& & & & & & & $-95 \%$ & $95 \%$ \\
\hline Xive & 37 & 2,38 & 2,00 & 1,00 & 5,00 & 1,19 & 1,98 & 2,77 \\
Semados & 25 & 2,32 & 2,00 & 1,00 & 5,00 & 1,35 & 1,76 & 2,88 \\
\hline Gesamt & 62 & 2,35 & 2,00 & 1,00 & 5,00 & 1,24 & 2,04 & 2,67 \\
\hline
\end{tabular}

Abbildung 3.17: Patientenbewertung des Halts der Prothese 


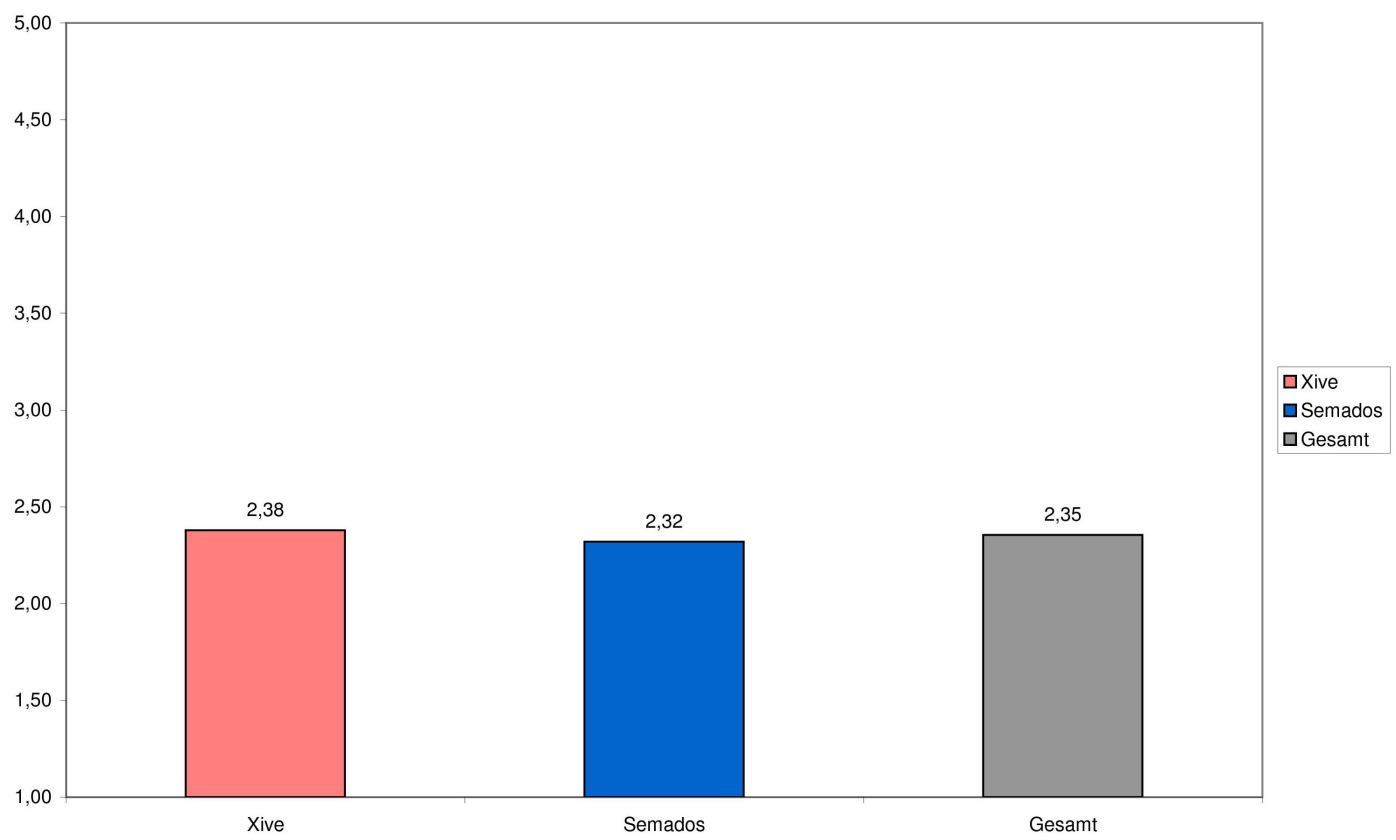

Abbildung 3.18: Halt der Prothese

\subsubsection{Bewertung der Kaufunktion}

Die Kaufunktion wurde von den insgesamt 62 Patienten mit einer Durchschnittsnote von 1,91 bewertet. Dabei wurde die Kaufunktion bei den Patienten mit Semadosimplantaten im Vergleich zu denen mit Xiveimplantaten etwas besser bewertet (siehe Abbildung 3.20). Im Mann-Whitney-U-Test konnte jedoch kein statistisch signifikanter Unterschied in der Bewertung der Kaufunktion der beiden Implantatsystemen festgestellt werden.

\begin{tabular}{|c|c|c|c|c|c|c|c|c|}
\hline & $\mathrm{N}$ & MW & Median & Min. & Max. & Std.abw. & $\begin{array}{c}\text { Konfidenz } \\
-95 \%\end{array}$ & $\begin{array}{c}\text { Konfidenz } \\
95 \%\end{array}$ \\
\hline Xive & 37 & 2,03 & 2,00 & 1,00 & 4,00 & 0,80 & 1,76 & 2,29 \\
\hline Semados & 25 & 1,80 & 2,00 & 1,00 & 4,00 & 0,82 & 1,46 & 2,14 \\
\hline Gesamt & 62 & 1,94 & 2,00 & 1,00 & 4,00 & 0,81 & 1,73 & 2,14 \\
\hline
\end{tabular}

Abbildung 3.19: Patientenbewertung der Kaufunktion 


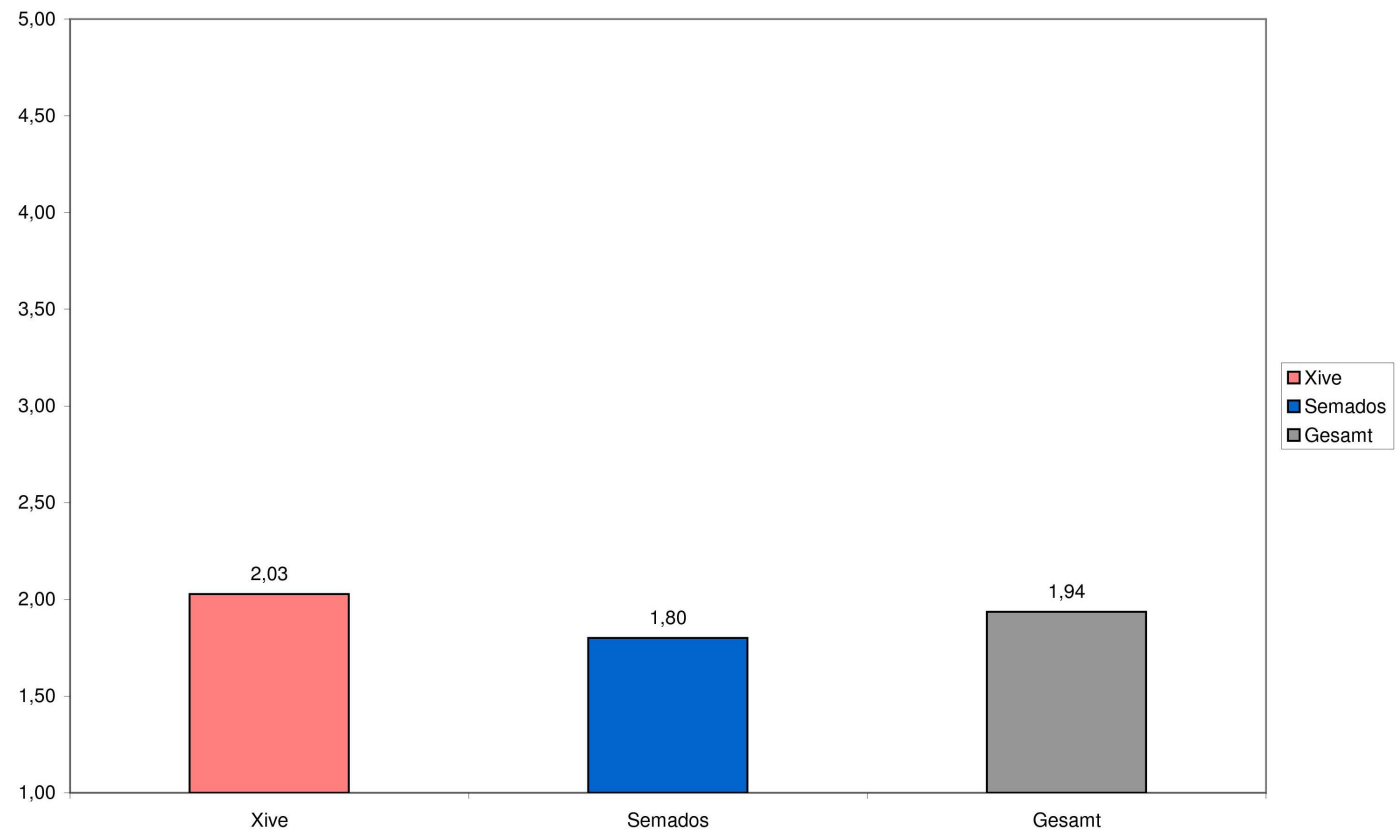

Abbildung 3.20: Kaufunktion (Mittelwerte)

\subsubsection{Bewertung der Reinigung der Prothese und Implantate}

Insgesamt wurde die Hygienefähigkeit der Deckprothese und der Implantate mit einer durchschnittlichen Note von 1,37 bewertet. Die Patienten mit Semadosimplantaten beurteilten die Hygienefähigkeit etwas besser als die Patienten mit Xiveimplantaten. Es konnten keine statistischen Unterschiede in Bezug auf die Hygienefähigkeit der beiden Implantatsysteme festgestellt werden.

\begin{tabular}{lcccccccc}
\hline & N & MW & Median & Min. & Max. & Std.abw. & $\begin{array}{c}\text { Konfidenz } \\
-95 \%\end{array}$ & $\begin{array}{c}\text { Konfidenz } \\
\end{array}$ \\
& 37 & 1,43 & 1,00 & 1,00 & 5,00 & 0,96 & 1,11 & 1,75 \\
\hline Xive & 25 & 1,28 & 1,00 & 1,00 & 2,00 & 0,46 & 1,09 & 1,47 \\
Semados & 62 & 1,37 & 1,00 & 1,00 & 5,00 & 0,79 & 1,17 & 1,57 \\
\hline Gesamt & & & & & &
\end{tabular}

Abbildung 3.21: Patientenbewertung der Reinigung der Prothese/Implantate 


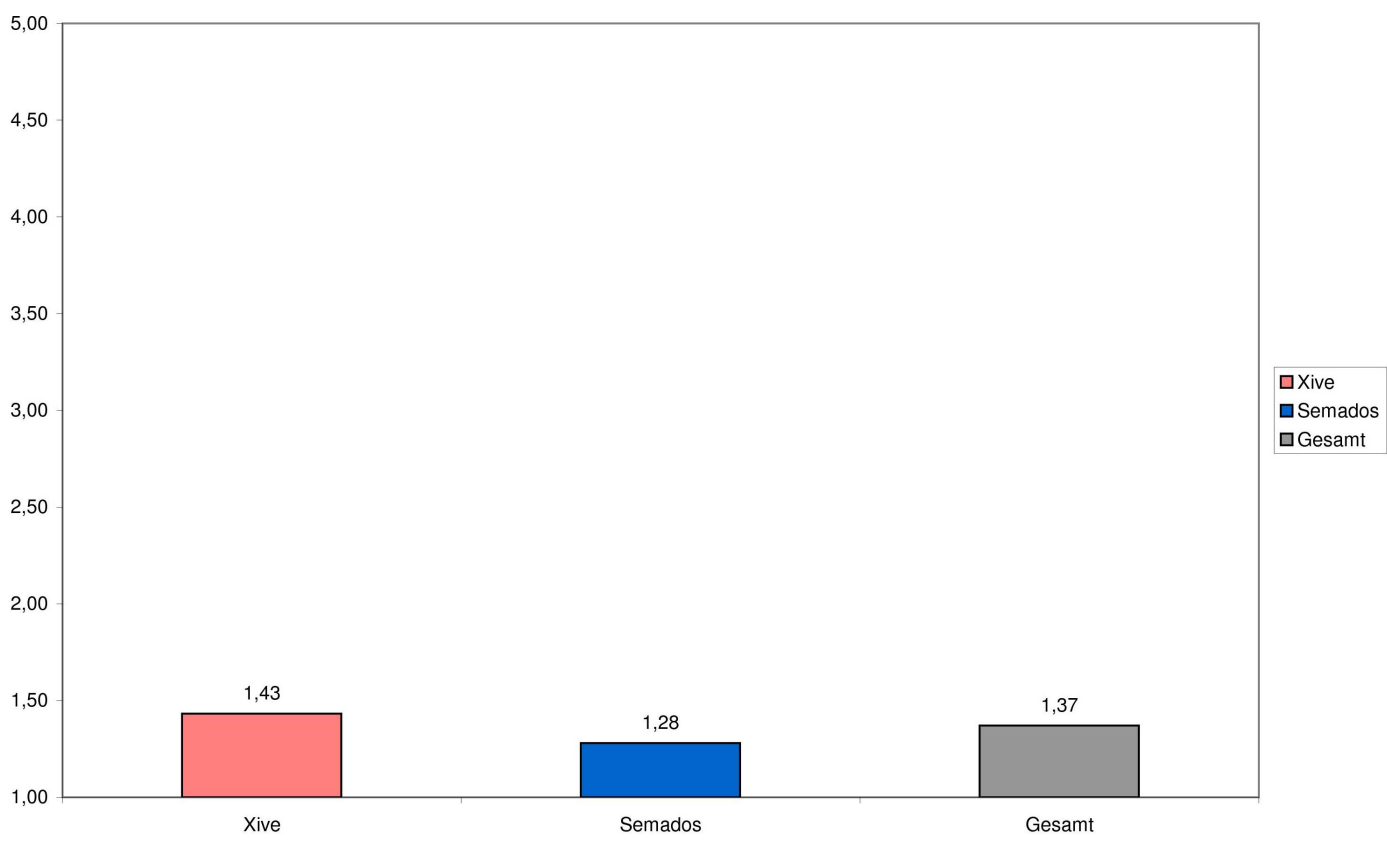

Abbildung 3.22: Reinigung der Prothese

\subsubsection{Anzahl der Zahnarztbesuche aufgrund von Komplikationen}

Die Patienten mussten aufgrund von Problemen mit ihrer Prothese oder den Implantaten im Mittel vier- bis fünfmal den Zahnarzt aufsuchen (siehe Tabelle 3.10) . Die Mediane zeigen jedoch, dass die meisten Patienten nur ein- bis zweimal behandlungsbedürftige Komplikation in einem mittleren Beobachtungszeitraum von 52 Monaten hatten. Die höheren Mittelwerte lassen sich dadurch erklären, dass bei sehr wenigen Patienten häufig Komplikationen auftraten und diese daher öfter den Zahnarzt aufsuchen mussten.

\begin{tabular}{lcccccccc}
\hline & N & MW & Median & Min. & Max. & Std.abw. & $\begin{array}{c}\text { Konfidenz } \\
-95 \%\end{array}$ & $\begin{array}{c}\text { Konfidenz } \\
95 \%\end{array}$ \\
\hline Xive & 37 & 4,32 & 1,00 & 0 & 37,0 & 7,70 & 1,76 & 6,89 \\
Semados & 25 & 4,92 & 2,00 & 0 & 50,0 & 11,2 & 0,32 & 9,52 \\
\hline Gesamt & 62 & 4,56 & 1,50 & 0 & 50,0 & 9,17 & 2,24 & 6,89 \\
\hline
\end{tabular}

Tabelle 3.10: Anzahl der Zahnarztbesuche 


\subsubsection{Nachfrage zur Behandlungsempfehlung des Göttinger Konzepts}

96,78 \% der befragten Patienten würden die Behandlung des Göttinger Konzepts an Familie, Freunde und Verwandte weiterempfehlen. Somit beantworteten nur 2 der 62 befragten Patienten diese Frage mit „nein“.

\subsubsection{Nachfrage zur Zufriedenheit mit der Mundgesundheit}

60 der 62 befragten Patienten gaben an, dass sie mit ihrer Mundgesundheit zufrieden seien. Jeweils ein Patient der Xive- und Semados-Gruppe beantworteten diese Frage mit „nein“. Folglich waren 96,78 \% der Patienten mit ihrer Mundgesundheit zufrieden.

\subsection{Auswertung des OHIP-Fragebogens}

Das Diagramm in Abbildung 3.23 zeigt die Mittelwerte der sieben Subskalen und des Totalpunktwertes. Die Punktwerte der einzelnen Subskalen ergeben sich aus den Summen der von den Patienten vergebenen Punkten auf der Likertskala (1-6). Ein hoher Punktwert ist somit Ausdruck für eine bessere Lebensqualität. Es fällt auf, dass in beiden Patientengruppen jede der Subskalen einen hohen Punktwert erreicht hat. In beiden Patientengruppen liegen die Mittelwerte der einzelnen Subskalen nah an den zu erreichenden Maximalpunkten (siehe Tabelle 3.11).

Die Semados-Gruppe bewertete insgesamt den OHIP-Fragebogen im Mittelwert mit 1,13 Punkten besser als die Xive-Gruppe. Bei der Durchführung des Mann-WhitneyU-Tests konnten jedoch keine statistisch relevanten Signifikanzen zwischen den beiden Gruppen festgestellt werden.

Die Mediane der Subskalen lagen nahe denen der jeweiligen Mittelwerte (siehe Tabelle 3.11), sodass nur geringe Schwankungen bei der Punktvergabe vorlagen. 


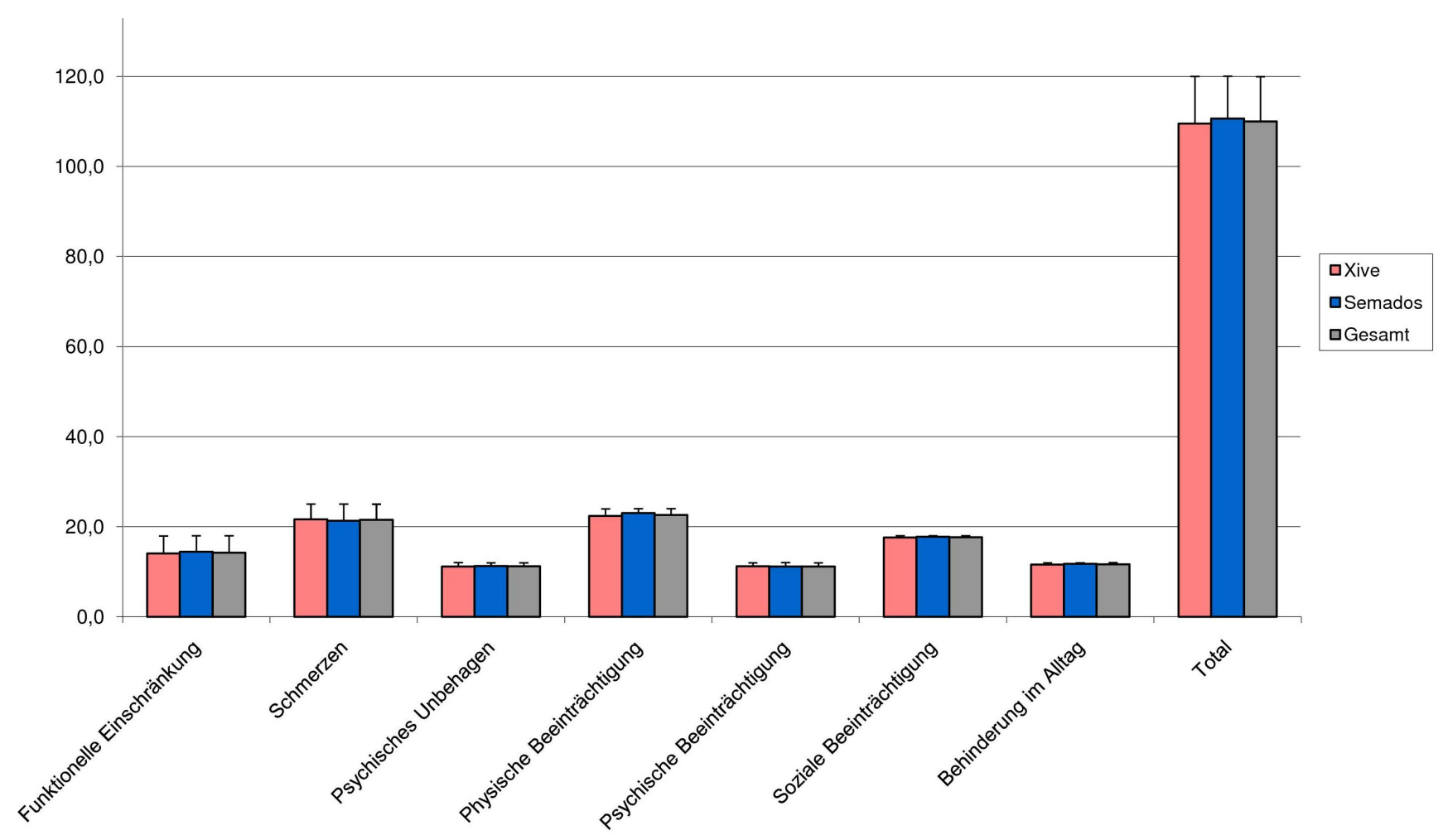

Abbildung 3.23: Mittelwerte der OHIP-Subskalen (Semados/Xive)

\begin{tabular}{|c|c|c|c|c|c|c|c|c|c|c|c|c|}
\hline & \multicolumn{5}{|c|}{ Semados } & \multicolumn{3}{|c|}{ Xive } & \multirow[b]{2}{*}{ Median } & \multirow[b]{2}{*}{ Min. } & \multirow[b]{2}{*}{ Max. } & \multirow[b]{2}{*}{ Std.abw } \\
\hline & $\mathrm{N}$ & MW & Median & Min. & Max. & Std.abw. & $\mathrm{N}$ & MW & & & & \\
\hline Funktionelle Einschränkung & 25 & 14,40 & 15,0 & 4,00 & 17,00 & 2,81 & 37 & 14,05 & 15,0 & 3,00 & 18,00 & 3,44 \\
\hline Schmerzen & 25 & 21,32 & 22,00 & 12,00 & 24,00 & 3,05 & 37 & 21,62 & 22,00 & 15,00 & 24,00 & 2,74 \\
\hline Psychisches Unbehagen & 25 & 11,28 & 12,00 & 8,00 & 12,00 & 1,21 & 37 & 11,14 & 12,00 & 6,00 & 12,00 & 1,55 \\
\hline Physische Beeinträchtigung & 25 & 23,00 & 24,00 & 16,00 & 24,00 & 1,85 & 37 & 22,35 & 24,00 & 12,00 & 24,00 & 2,69 \\
\hline Psychische Beeinträchtigung & 25 & 11,12 & 12,00 & 8,00 & 12,00 & 1,24 & 37 & 11,19 & 12,00 & 5,00 & 12,00 & 1,79 \\
\hline Soziale Beeinträchtigung & 25 & 17,76 & 18,00 & 16,00 & 18,00 & 0,60 & 37 & 17,59 & 18,00 & 12,00 & 18,00 & 1,12 \\
\hline Behinderung im Alltag & 25 & 11,76 & 12,00 & 9,00 & 12,00 & 0,72 & 37 & 11,57 & 12,00 & 6,00 & 12,00 & 1,28 \\
\hline OHIP-Gesamtwert & 25 & 110,64 & 114,00 & 73,00 & 119,00 & 9,57 & 37 & 109,51 & 114,00 & 75,00 & 120,00 & 12,11 \\
\hline
\end{tabular}

Tabelle 3.11: Ergebnisse des OHIP - Fragebogens 


\subsection{Falldokumentation einer Patientin}

Abbildung 3.24 zeigt die OPAN-Aufnahme einer 59-jährigen Patientin aus dem Jahr 2002. Diese war seit neun Jahren zahnlos und prothetisch im Ober- und Unterkiefer mit einer Totalprothese versorgt. Die Patienten klagte trotz wiederholter Unterfütterung ihrer Unterkieferprothese über einen starken Retentionsverlust. Im November 2002 wurden der Patientin im Rahmen dieser prospektiven Studie zwei Implantate (Semados, Bego, Bremen) in Regio 033 und 043 inseriert (siehe Abbildung 3.25). Um eine sofortige Belastung der Implantate zu ermöglichen wurden die Implantate durch zusätzliche Satellitenimplantate stabilisiert (Regio 033 mesial/distal, Regio 043 distal). Die beiden Implantate wurden anschließend mit Kugelattachments versorgt. Am gleichen Tag erfolgte eine Umarbeitung der Uk-Prothese, um die beiden Matrizen zu integrieren (siehe Abbildung 3.26). Drei Monate nach der Implantation und einer erfolgreich abgeschlossenen Osseointegration der Implantate wurden die Satellitenimplantate wieder entfernt.

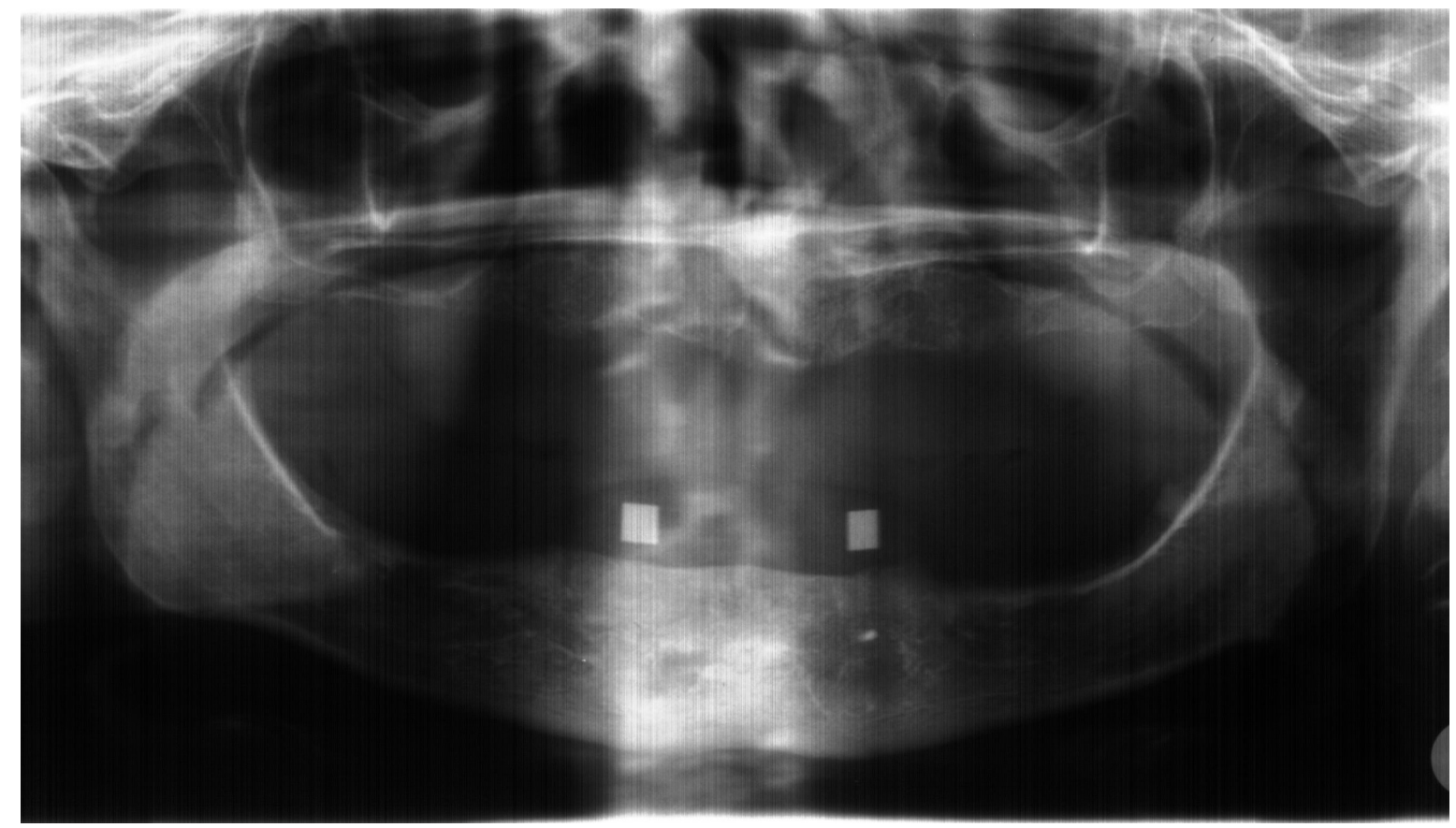

Abbildung 3.24: Orthopantomogramm mit quadrangulärem Referenzkörper Regio 033 und 043 


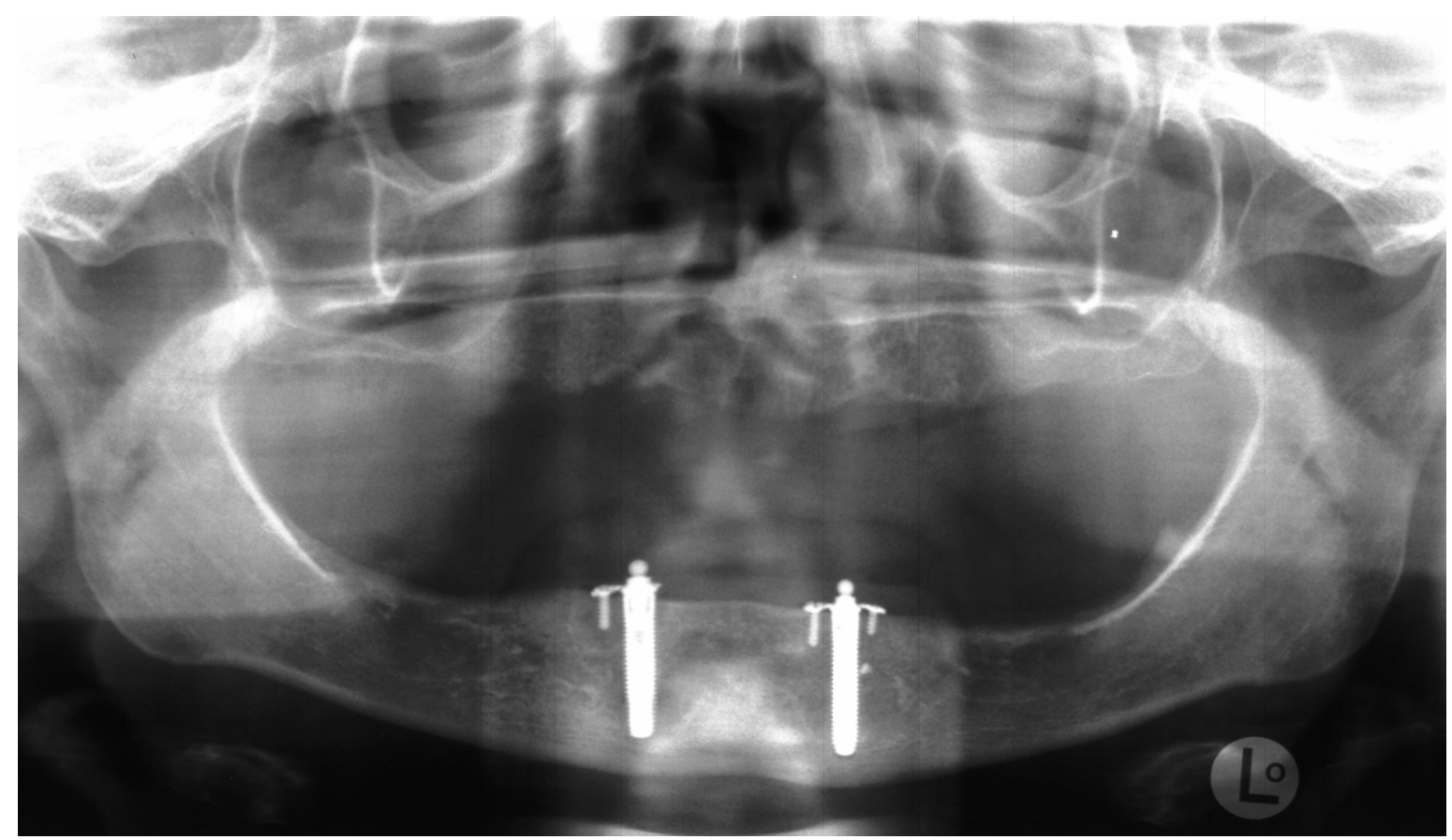

Abbildung 3.25: Orthopantomogramm nach Implantation mit Satellitenimplantaten

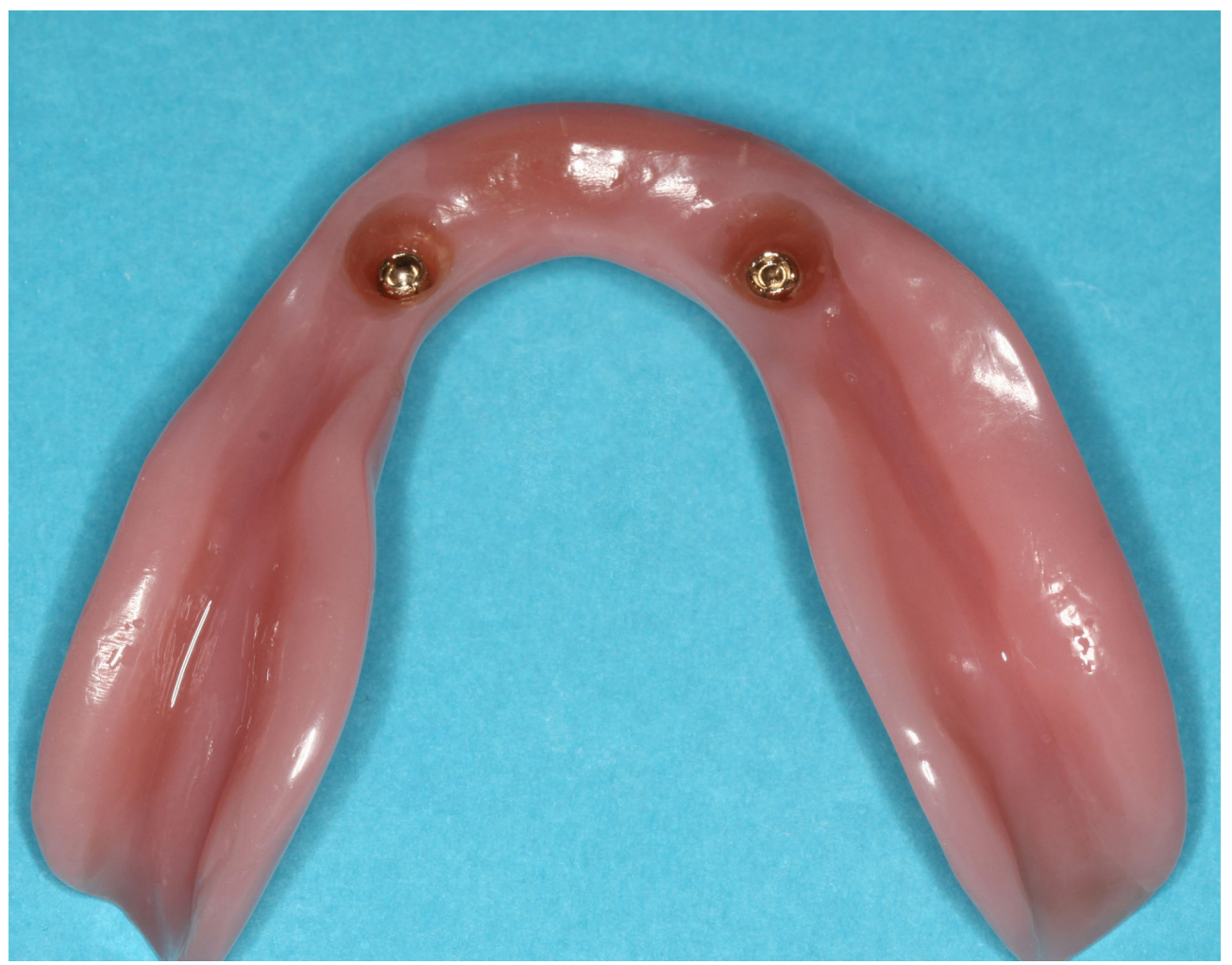

Abbildung 3.26: Umgearbeitete UK-Prothese mit Matrizen 


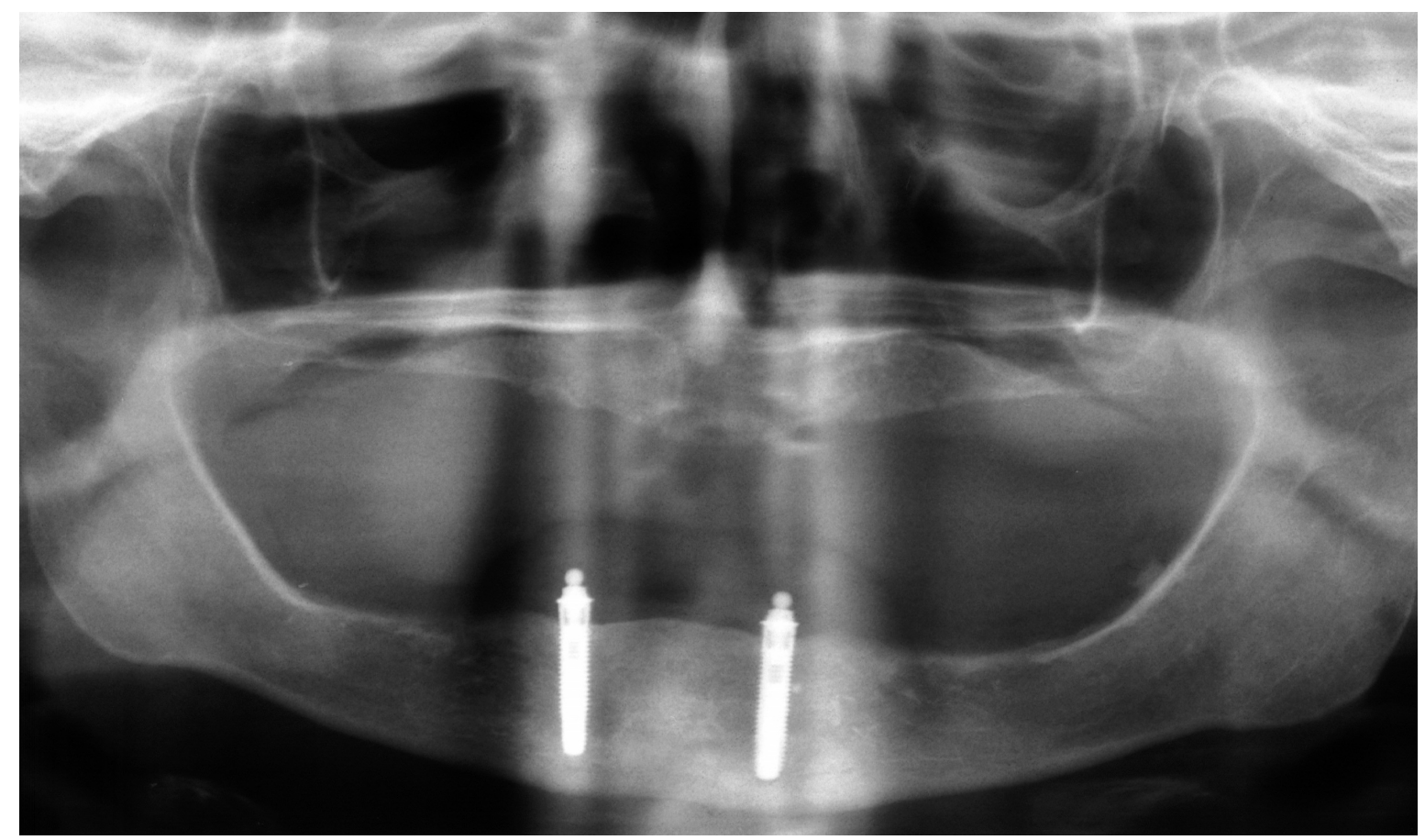

Abbildung 3.27: Orthopantomogramm sechs Jahre nach Implantation

Abbildung 3.27 zeigt eine OPAN-Aufnahme der Patientin sechs Jahre nach der Implantation. Der horizontale Knochenabbau betrug an Implantat 033 mesial 0,7 mm/ distal 0,8 mm und Implantat 043 mesial 1,1 mm/ distal 1,0 mm. Die Messung der Implantatstabilität mit dem Periotestgerät ergab an Implantat $033-5$ und $043-7$.

Abbildung 3.28 zeigt die klinische Situation der Patientin im Unterkiefer. Die periimplantäre Gingiva weist keine Reizungen auf. Es bestehen keine klinischen Anzeichen einer Periimplantitis. Die Sondierungstiefen wurden an Implantat 033 und 043 kleiner als 2,5 mm gemessen. Die Retention der UK-Prothese wurde durch die Kugelattachments wesentlich verbessert. Die Patientin bewertete die Retention der Prothese nach dem deutschen Schulnotensystem mit „gut“ und das gesamte Behandlungsresultat mit ,sehr gut". 


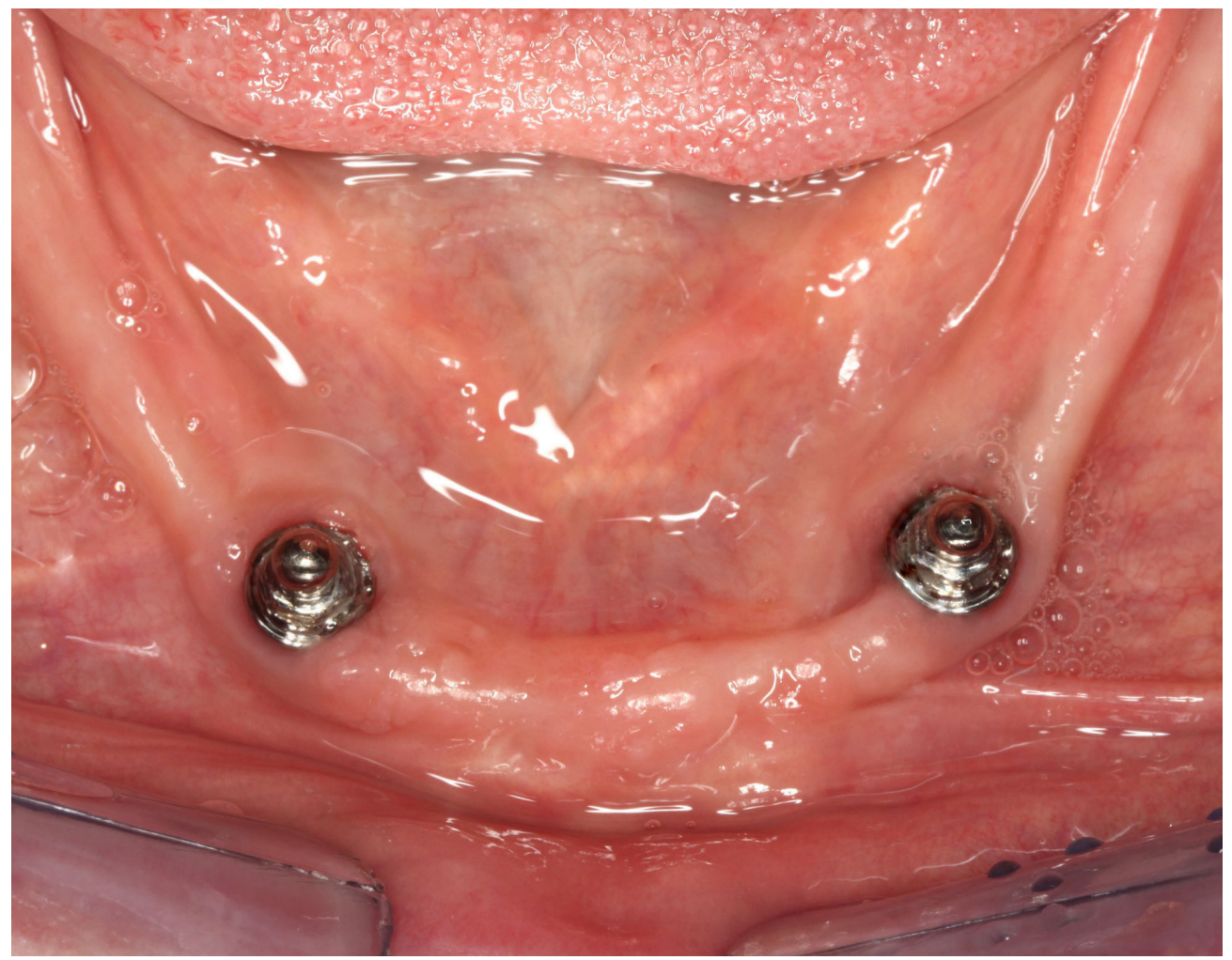

Abbildung 3.28: Intraorale Aufnahme 6 Jahre nach Implantation

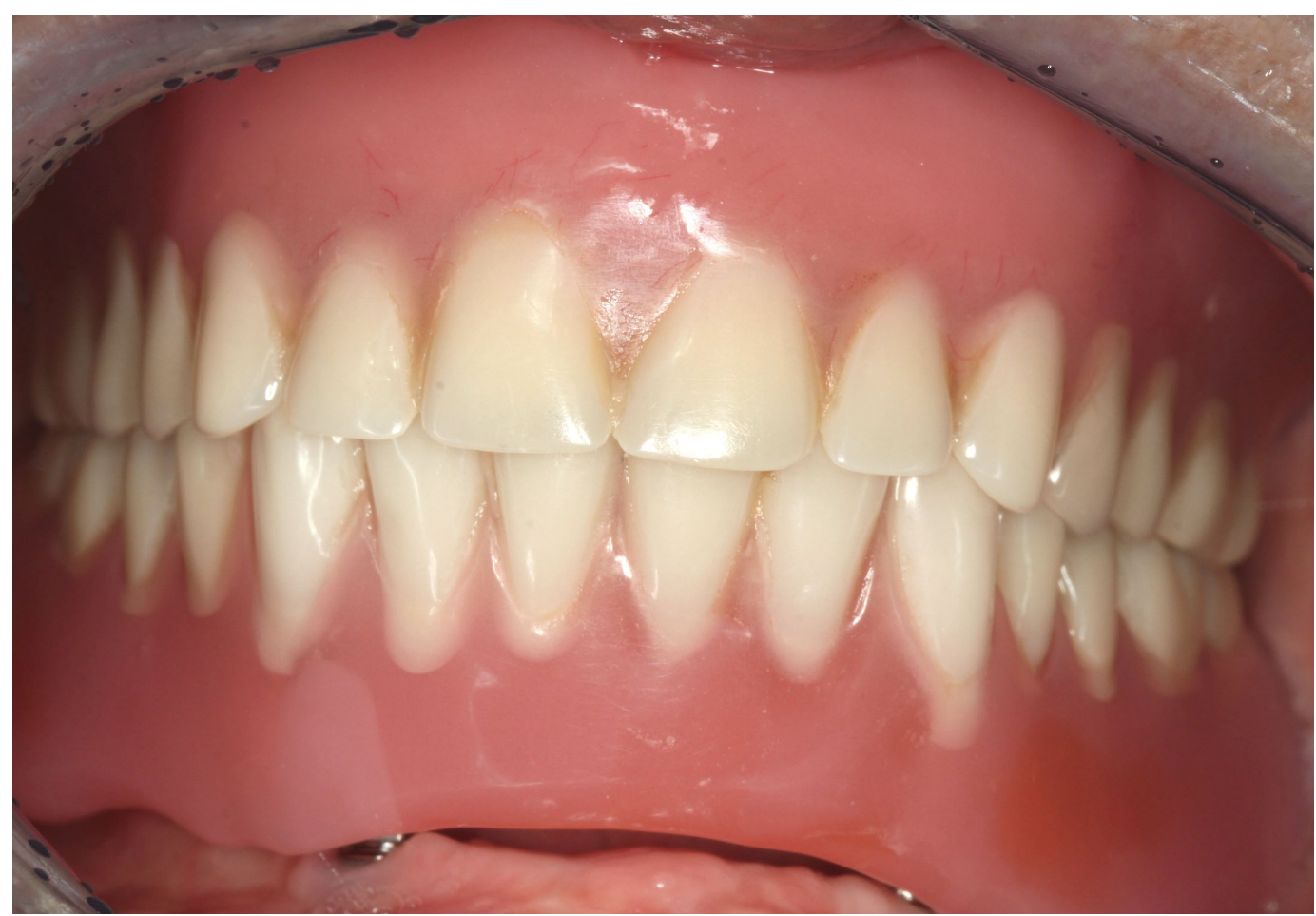

Abbildung 3.29: Prothese in Okklusion 


\section{Diskussion}

\subsection{Beurteilung der Überlebenszeitanalyse und Erfolgsquote}

Die Beurteilung einer erfolgreichen dentalen Implantation wird bis heute kontrovers diskutiert. Als anerkanntes Standardverfahren zur reinen Analyse der Überlebensstatistik gilt die Erfolgskurve nach Kaplan und Meier (1958), welche schon auf der Konsensuskonferenz der DGZMK 1989 (Tetsch et al. (1990)) für unverzichtbar erklärt wurde.

Die Überlebensstatistik dieser Studie betrug nach Kaplan und Meier (1958) insgesamt 97,28 \%. Das heißt, dass in einem mittleren Beobachtungszeitraum von 52 Monaten von den insgesamt 172 inserierten Implantaten fünf Verluste auftraten. Die getrennte Betrachtung der Überlebensstatistik für die beiden Implantatsysteme ergab für das Xive-Implantat 95,19 \% und das Semados-Implantat 100 \%. Dabei fiel auf, dass die Verluste nur bei den Patienten mit Xive-Implantaten auftraten. Es erweist sich allerdings als schwierig, die Ursache für diesen signifikanten Unterschied auszumachen, da beide Implantatsysteme sehr ähnliche Eigenschaften besitzen. In mehreren Studien konnte gezeigt werden, dass die Verlustrate bei Implantaten mit modifizierten Oberflächen im Vergleich zu maschinierten Titanoberflächen geringer war (Buser et al. (1998), Bruyn und Collaert (2002), Glauser et al. (2003)) Die Adhäsion von Zellen an Implantatoberflächen spielte hierbei eine entscheidende Rolle, da raue Oberflächen von osteogenen Zellen bevorzugt besiedelt wurden (Kieswetter et al. (1996)). Jedoch handelt es sich bei beiden Implantatsystemen dieser Studie um Implantate mit einer gestrahlten und geätzten Oberfläche, so dass die Oberflächenbeschaffenheit der Implantate als Ursache für dieses Phänomen unwahrscheinlich ist.

Um den Einfluss des Geschlechts und des Alters der Patienten auf die Überlebensrate der Implantate zu überprüfen, wurde außerdem eine Kovarianzanalyse durchgeführt. Es konnte jedoch kein statistisch signifikanter Zusammenhang festgestellt werden.

Vergleicht man die Überlebensrate dieser Studie mit denen in der aktuellen Literatur, so finden sich ähnliche Ergebnisse mit Implantatüberlebensraten von über 95 \% (Brånemark et al. (1999) 98\%, Chiapasco und Gatti (2003) 96,1 \%, Degidi et al. (2007) 100 $\%$, Marzola et al. (2007) $100 \%$, Stoker und Wismeijer (2009) 98,8\%). Allerdings sind 
diese Resultate aufgrund der höheren Implantatzahl und der Stegkonstruktion nicht direkt mit den Ergebnissen dieser Arbeit vergleichbar. In den meisten Studien, die eine Sofortbelastung durch Deckprothesen im Unterkiefer anstrebten, wurde zur Erhöhung der Primärstabilität eine Verblockung der Implantate durch eine Stegkonstruktion vorgenommen. Dabei wurden bei jedem Patienten mindestens drei bis vier Implantate in der interforaminalen Region inseriert (Alfadda et al. (2009), Assad et al. (2007), Stoker und Wismeijer (2009), Brånemark et al. (1999)). In diesem Zusammenhang haben die hohen Erfolgsraten gezeigt, dass eine Sofortbelastung verblockter Implantate ohne Gefährdung einer erfolgreichen Osseointegration durchzuführen ist. Es gibt nur wenige Publikationen, die sich mit einer Sofortbelastung von nur zwei interforaminalen Implantaten mit Kugelattachments beschäftigt haben. Ähnliche Studien zu dieser Arbeit wurden von Ormianer et al. (2006) und Marzola et al. (2007) durchgeführt. Dabei wurden zwei interforaminale Implantate im Unterkiefer inseriert. Trotz einer fehlenden Verblockung berichteten die Autoren von einer Überlebensraten von über $95 \%$. Die hohen Erfolgsraten dieser Studien wurden durch den Implantattyp mit einer osseokonduktiven Oberfläche begründet. So führte die Verwendung solcher Oberflächen zu einer raschen und gesteigerten Knochenanlagerung am Implantat und ermöglichte dadurch in kurzer Zeit eine erfolgreiche Osseointegration .

Bei der reinen Überlebensstatistik nach Kaplan und Meier (1958) werden verschiedene klinische Parameter nicht berücksichtigt. Die Frage nach der funktionellen Nutzung des Implantats sowie der Zustand der periimplantären Weich- und Hartgewebe werden nicht beantwortet. Deshalb haben in den vergangenen Jahren verschiedene Autoren zusätzliche Erfolgskriterien entwickelt und mit in ihre Studien aufgenommen (Schnitman und Shulman (1980), Albrektsson et al. (1986), Buser et al. (1990), Jahn und d'Hoedt (1992), Naert et al. (1992)). Allerdings existiert bis heute kein einheitlicher internationaler Konsens, der einen differenzierten Vergleich der Arbeiten erlaubt. Für diese Studie wurden die Kriterien nach Jahn und d'Hoedt (1992) und Naert et al. (1992) ausgewählt, da diese zusätzlich die subjektive Bewertung des Patienten sowie die prothetische Versorgung des Implantats mit einbeziehen.

Die Erfolgsquote nach Jahn und d'Hoedt (1992) betrug 95,11 \% und die nach Naert et al. (1992) 97,28 \%. Die unterschiedlichen Ergebnisse resultieren aus den verschiedenen Erfolskriterien. Bei Jahn und d'Hoedt (1992) wird zusätzlich die subjektive Benotung des Implantats durch die Patienten berücksichtigt. Da vier Patienten das Ergebnis schlechter als „befriedigend“ bewerteten, resultierte daraus eine niedrigere Erfolgsquote. Chiapasco und Gatti (2003) berichteten von einer ähnlichen Erfolgsrate von 91,6 \% bei sofortbelasteten interforaminalen Implantaten. 


\subsection{Beurteilung der Implantatstabilität}

Die Implantatstabilität wird als eines der wichtigsten Kriterien zum Erreichen und Erhalten einer erfolgreichen Osseointegration angesehen (Brånemark (1983), Glauser et al. (2003)). Daher gehört es heute mit zur Routine, nach der Insertion des Implantats die primäre Stabilität zu bestimmen. Zu den am weitesten verbreiteten nicht-invasiven Methoden gehören dabei die Resonanz Frequenz Analyse (Osstell Integration Diagnostics AB, Sävedalen, Schweden) und das Periotestgerät (Medizintechnik Gulden, Bensheim). In dieser Studie wurden die Messungen mit dem Periotestgerät durchgeführt. Aparicio (1997) empfahl nach einer Studie mit 315 Patienten und einer Gesamtzahl von 1182 Implantaten, die Verwendung von Periotestwerten als erstes Erfolgskriterium nach einer Implantation. Noguerol et al. (2006) bezeichneten ebenfalls die Periotestwerte als einen guten Prädiktor, um einen eventuellen frühen Implantationsmisserfolg festzustellen. Ihrer Ansicht nach hätten diese auch eine höhere Spezifität als eine röntgenologische Auswertung. Das Periotestgerät eignet sich jedoch nicht nur zur Bestimmung der primären Stabilität direkt nach der Implantation, sondern auch zur Bestimmung der sekundären Stabilität bei der klinischen Nachuntersuchung der Patienten (Engelke et al. (2005)).

Allerdings konnte in verschiedenen Studien gezeigt werden, dass bestimmte Faktoren die Messung des Periotests beeinflussen können. Meredith et al. (1998) beschrieben eine lineare Abhängigkeit der Periotestwerte vom vertikalen Abstand des Messpunktes am Abutment bis zum ersten Knochenkontakt. Aus diesem Grund würden sich die Periotestwerte nicht zur direkten Messung der Implantat-Knochen-Verbindung eignen. Folglich ließen Einzelmessungen keine Prognose über die Implantatstabilität zu. Lediglich durch mehrere Messungen während einer Nachuntersuchung könnten Aussagen über die Implantatstabilität gemacht werden. Einen weiteren Einflussfaktor stelle der Winkel des Handstücks zum Abutment dar. Olivé und Aparicio (1990) beschrieben Differenzen der Periotestwerte von 1-2, bei Veränderung des Winkels und der Höhe des Messpunktes am Abutment.

In dieser Studie wurden im Rahmen der Nachuntersuchung bei 62 Patienten nach einer mittleren Beobachtungszeit von 52 Monaten an 120 Implantaten die Periotestwerte bestimmt. Insgesamt betrug der Mittelwert für beide Implantatsysteme - 4,86. Bis auf eine Ausnahme (PTW +3 ) wurden an allen anderen Implantaten negative Periotestwerte gemessen. Dies ist ein Hinweis auf eine hohe Stabilität der Implantate, die auf eine erfolgreiche Osseointegration schließen lässt. Der Mittelwert für die Patienten mit Semadosimplantaten betrug -5,27. Bei den Patienten mit Xive-Implantaten wurde hingegen ein Wert von -4,58 gemessen. Trotz dieser Differenz konnten keine signifikanten Unterschiede zwischen den beiden Implantatsystemen ermittelt werden ( p-Wert 0,23). Auch die Position und das Alter der Patienten hatte keinen Einfluss auf die gemessen 
Periotestwerte.

Allerdings konnte ein Zusammenhang zwischen der Verweildauer der Implantate im Kiefer und der Periotestwerte erkannt werden. Mit der Zeit nahmen die Werte kontinuierlich ab. Ledermann et al. (1998), Schenk und Buser (1998) und Smet et al. (2007) beobachteten das gleiche Phänomen. Dabei konnten Schenk und Buser (1998) durch den Vergleich histologischer Befunde von 1985 mit denen von 1998 zeigen, dass die Osseointegration von den sofortbelasteten interforaminalen Implantaten in der Mandibula unter der vieljährigen Belastung nicht nur erhalten bleibt, sondern über die Jahre sogar noch zugenommen hat. Dies ließ sich auch klinisch durch die abnehmenden Periotestwerte in den Nachuntersuchungen verifizieren (Ledermann et al. (1998)).

Vergleicht man die Periotestwerte dieser prospektiven Studie mit denen aktueller Arbeiten, so sind ähnliche Ergebnisse zu finden. Liao et al. (2010) berichteten über mittlere Periotestwerte bei sofortbelasteten interforaminalen Implantaten von -4,25 nach einem Jahr. Auch Smet et al. (2007) stellten vergleichbare Ergebnisse in einer Arbeit über sofortbelastete, frühbelastete und spätbelastete unverblockte Implantate in der anterioren Mandibula vor. Die mittleren Periotestwerte wurden nach 12 Monaten für die Gruppe der sofortbelasteten Implantate mit -3,67 angegeben. Auch Kawai und Taylor (2007) veröffentlichten in einer systematischen Literaturübersicht über sofortbelastete Implantate durch Deckprothesen mittlere Periotestwerte von -4,6 nach 24 Monaten. Im Rahmen dieser Studie zeigen die Periotestwerte tendenziell negativere Werte als die Arbeiten in der aktuellen Literatur. Zudem ist der Nachuntersuchungszeitraum von 52 Monaten wesentlich größer und lässt daher auch Rückschlüsse auf die Nachhaltigkeit und den längerfristigen Erfolg der Sofortbelastung mit kortikaler Mikrofixation zu.

\subsection{Beurteilung des Knochenabbaus}

Abgesehen von der reinen Überlebensstatistik und der Stabilität des Implantats stellt der radiologisch gemessene periimplantäre Knochenabbau einen weiteren wichtigen Parameter für den Implantaterfolg dar. Dabei ist bei jeder Implantation mit einem geringen Knochenabbau zu rechnen. Als Hauptgrund wird heute der Mikrogap zwischen Implantat und Abutment angenommen, der sowohl durch eine erhöhte mikrobielle als auch mechanische Belastung den Abbau von Knochen um das Implantat fördert. So berichteten einige Autoren von einem Zusammenhang zwischen dem periimplantären Knochenabbau und der Qualität und Quantität der mikrobiellen Besiedlung des Mikrogaps (Apse et al. (1989), Hultin et al. (2000)). Außerdem wirken Abutment und Suprakontruktion als Hebelarm, welcher an der Implantatschulter ansetzt und besonders am Mikrogap zu einer starken mechanischen Spannung führt. In einer Studie von King et al. (2002) konnte durch eine starre Verbindung zwischen Implantat und Abutment 
durch Verschweißen, ein geringerer Knochenverlust im Vergleich zur Kontrollgruppe erzielt werden. Den größten Einfluss auf den Erfolg einer Implantation und auch das Ausmaß des Knochenabbaus hat jedoch immer noch das individuelle Können und die Erfahrung des Chirurgen und Prothetikers (Albrektsson (2001)).

Zur Beurteilung und Einschätzung des fortschreitenden Knochenverlusts nach einer Implantation wurden von vielen Autoren verschiedene Richtlinien und Empfehlungen vorgeschlagen. Schon sehr früh formulierten Schnitman und Shulman (1979) auf der NIH-Konferenz Mindestkriterien für eine erfolgreiche Implantation. In Bezug auf den Knochenverlust sei dabei ein periimplantärer Knochenabbau geringer als $1 / 3$ der Implantatlänge als Erfolg zu werten. Albrektsson et al. (1986) legten als Mindestkriterium einen jährlichen Knochenabbau von maximal 0,2 mm um das inserierte Implantat nach dem ersten Jahr prothetischer Belastung fest. Nach einer Stellungnahme der DGZMK (2000) wird ein Knochenabbau von $0,5 \mathrm{~mm}$ nach dem ersten Jahr nach Implantation und in den folgenden Jahren von 0,1-0,2 mm als vertretbar angesehen.

In dieser Studie wurde der horizontale Knochenabbau mit einem digitalen Vermessungsverfahren bestimmt. Die Vorteile im Gegensatz zur analogen Bestimmung durch eine Messschablone oder eines Lineals bestehen in der höheren Ablesegenauigkeit sowie der individuellen Umrechnung des Vergrößerungsfaktors für jede einzelne Röntgenaufnahme durch die Computersoftware (Sidexis XG,Sirona). Durch die bekannte Implantatlänge als Referenz ist es nicht erforderlich, einen allgemeinen Vergrößerungsfaktor von 1,25 bei jeder Aufnahme anzunehmen. Dennoch ist auch bei dieser Methode mit einer gewissen Messungenauigkeit zu rechnen. Grundsätzlich stellt ein Röntgenbild ein zweidimensionales Abbild eines dreidimensionalen Objekts dar. Es entsteht ein Summationsbild der Objekte innerhalb eines Strahlengangs. Dabei kommt es zu Überlagerungen der abgebildeten Strukturen, die zudem eine unterschiedliche Röntgendichte aufweisen. Dies kann dazu führen, dass feinere Strukturen überlagert und nicht abgebildet werden. Vor diesem Hintergrund scheint es fragwürdig, die geforderte jährliche Vermessung des Knochenverlusts im Bereich von 0,2 mm auf der Basis der Orthopanthomogramme überhaupt technisch durchführen zu können (Buch et al. (2003)). Gómez-Román et al. (1995) sehen daher nur eine akzeptable Messgenauigkeit von $1 \mathrm{~mm}$ als realisierbar. Dennoch wurde in dieser Studie versucht, den Knochenabbau so präzise wie möglich anzugeben. Ein Messbereich von $0,5 \mathrm{~mm}$ wurde als angemessen festgelegt. Möglicherweise hätte durch die Verwendung eines digitalen Orthopantomographen ein genaueres Messergebnis erzielt werden können. Da ein solches Gerät im Zeitraum der Nachuntersuchungen nicht zur Verfügung stand, war dies leider nicht möglich.

In dieser Studie ergab die Auswertung der radiologischen Parameter für beide Implantatsysteme nach einem mittleren Beobachtungszeitraum von 52 Monaten einen mittleren Knochenverlust von 1,12 mm mesial und 0,99 mm distal. Für das Xive-Implantat 
betrug dieser 1,18 mm mesial und 1,09 mm distal und für das Semados-Implantat 1,04 mesial und 0,85 mm distal. Bezieht man diese Werte auf die zuvor genannte Stellungnahme der DGZMK (2000) sowie die Erfolgskriterien nach Albrektsson et al. (1986), Jahn und d'Hoedt (1992) und Naert et al. (1992) so werden diese Richtlinien für beide Implantatsysteme erfüllt.

In dieser Studie wurden zur genaueren Analyse der erhobenen radiologischen Parameter zudem eine Kovarianzanalyse (ANCOVA) durchgeführt. Dabei wurden mögliche zusätzliche Einflussfaktoren auf den horizontalen Knochenverlust getestet. In diesem Zusammenhang konnte ein signifikanter Einfluss der Verweildauer der Implantate auf den distalen Knochenabbau sowie ein tendenzieller Einfluss auf den mesialen Knochenabbau festgestellt werden. Dieses Ergebnis war zu erwarten da, immer ein gewisser Knochenabbau innerhalb der ersten 5 Jahre nach Implantation stattfindet und als physiologisch angesehen werden kann. Fünf Jahre nach der Implantation bleibt dieser allerdings nach Ansicht einiger Autoren relativ stabil (Lambrecht et al. (2003),Astrand et al. (2004)).

Die Kovarianzanalyse zeigte außerdem einen signifikanten Unterschied der beiden Implantatsysteme auf den distalen Knochenverlust $(\mathrm{p}=0,03)$. Bei den Patienten mit XiveImplantaten wurde distal ein wesentlich größerer Knochenverlust als bei den Patienten mit Semados-Implantaten festgestellt (Xive $=1,09 \mathrm{~mm}$, Semados $=0,85 \mathrm{~mm}$ ). Mesial konnte zwar auch ein größerer Knochenverlust bei Patienten mit Xive-Implantaten gemessen werden $($ Xive $=1,19 \mathrm{~mm}$, Semados $=1,04 \mathrm{~mm}$ ), allerdings waren diese Werte nicht signifikant $(\mathrm{p}=0,18)$.

Eine wichtige Frage, die sich im Rahmen dieser Studie nach der Entfernung der Satellitenimplantate stellte, war der Einfluss dieser kortikalen Schrauben auf den weiteren Verlauf des periimplantären Knochenabbaus. Engelke et al. (2005) berichteten in einer ähnlichen Studie mit einer Satellitenimplantation von einem mittleren Knochenabbau von $0,7 \mathrm{~mm}$ nach einer mittleren Beobachtungszeit von 10 Monaten. Dieses Ergebnis war vergleichbar mit denen vieler anderer Autoren (Adell et al. (1981), Quirynen et al. (1991)), erfüllte allerdings nicht die Kriterien der Stellungnahme der DGZMK (2000) (Knochenverlust max. 0,5 mm im ersten Jahr nach Implantation). Dieser anfangs leicht erhöhte Knochenabbau könnte möglicherweise auf die Remodellierungsprozesse am krestalen Knochen nach Entfernung der Satellitenimplantate zurückzuführen sein. Jedoch zeigen die Ergebnisse dieser Studie nach 52 Monaten, dass die Verwendung kortikaler Schrauben zur Fixierung der Zentralimplantate keinen negativen Einfluss auf den periimplantären Knochenverlust gehabt hat.

Die gemessen Werte sind im Vergleich zu denen aktueller Studien der Sofortbelastung kleiner oder annähernd gleich groß. Kawai und Taylor (2007) berichteten in einer Literaturübersicht mit Metaanalyse von einem mittleren Knochenabbau von 1,2 mm nach 
24 Monaten. Bei einer prospektiven Studie von Smet et al. (2007) wurde nach 12 Monaten schon ein mittlerer Knochenabbau von 1,63 mm gemessen. Auch Turkyilmaz et al. (2006) veröffentlichten bei sofortbelasteten unverblockten Implantaten Werte von 1,13 mm nach 24 Monaten. Es gibt jedoch momentan keine Studien sofortbelasteter interforaminaler Implantate mit Deckprothesen, die Ergebnisse über den periimplantären Knochenabbau in einem ähnlich langen Beobachtungzeitraum liefern.

\subsection{Beurteilung der Sondierungstiefen}

Zur Diagnostik der periimplantären Weichgewebe wurden bei allen Patienten dieser Studie die Sulkustiefen mesial und distal am Implantat bestimmt. Die Sondierung der Taschen ist ein weiteres wichtiges diagnostisches Mittel, um frühzeitig eine entzündliche Veränderung um das Implantat erkennen zu können. Denn besonders bei der periimplantären Gingiva sind die klassischen Entzündungszeichen wie Schwellung und Rötung wesentlich schwächer ausgeprägt als beim Parodontium, da nur eine Durchblutung vom Periost des umgebenden Knochens her erfolgt. Die parodontale Gingiva hingegen wird sowohl von supraperiostalen Gefäßen als auch von denen des parodontalen Ligamentes versorgt (Berglundh et al. (1994)). Daher ist eine reine Inspektion und radiologische Diagnostik der Implantate nicht ausreichend und veranlasste verschiedene Autoren dazu, diesen Untersuchungsparameter als ein weiteres wichtiges Erfolgskriterium für eine Implantation aufzunehmen (Jahn und d'Hoedt (1992), Spiekermann et al. (1995)).

Die Erhebung der Sondierungstiefen birgt allerdings auch einige Risiken. Das epitheliale Attachment am Implantat ist nur gering ausgebildet. Daher können leicht bei der Sondierung des periimplantären Weichgewebes Verletzungen hervorgerufen werden. Histologische Untersuchungen von Schou et al. (2002) haben gezeigt, dass bei einem Sondierungsdruck von 0,5 N das Barrierenepithel der periimplantären Schleimhaut durch die Sonde penetriert werden konnte und diese bis zu einer Distanz von 0,2 $\mathrm{mm}$ an den marginalen Knochen heranreichte. Bei einem Sondierungsdruck von 0,2 N bis $0,3 \mathrm{~N}$ war dies nicht möglich. Zudem kann auch durch die Parodontalsonde das tiefergelegene periimplantäre Gewebe mit parodontalen Keimen kontaminiert werden. Um diese Risiken zu minimieren, wurde die Untersuchung immer vom selben Behandler und mit der gleichen Sonde durchgeführt und bei der Sondierung der Taschen auf einen adäquaten Druck geachtet.

Die Ergebnisse dieser Studie ergaben für die Messung der periimplantären Sondierungstiefen für beide Implantatsysteme mittlere Sondierungstiefen von 2,46 mm. Für die Patienten mit Xive-Implantaten betrugen die Mittelwerte 2,45 mm. Für die Patienten mit Semados-Implantaten wurden mittlere Sondierungstiefentiefen von 2,47 mm gemessen. Es konnte bei der Kovarianzanalyse dieser Werte ein signifikanter Zusam- 
menhang zwischen der Verweildauer der Implantate im Kiefer und den distalen Sondierungstiefen festgestellt werden. Je länger die Implantate in situ waren, desto kleiner waren die gemessenen Taschentiefen. Dieses Phänomen konnte tendenziell auch für die mesial am Implantat gemessenen Werte beobachtet werden. In Studien von Chiapasco et al. (2001), Romeo et al. (2002) sowie Chiapasco und Gatti (2003) wurde ebenfalls von einer solchen Tendenz berichtet.

Bei der Sondierung traten an sechs Implantaten kleinere Blutungen auf. Dabei war auffällig, dass alle Blutungen an Xive-Implantaten vorkamen und ein signifikanter Zusammenhang zwischen den Blutungen und dem Implantatsystem nachgewiesen werden konnte $(\mathrm{p}=0,008)$. Diese kleineren Blutungen sind als Reizung der periimplantären Gingiva zu werten. Die Sondierungstiefen überschritten jedoch bei keinem Patienten den kritischen Wert von $4 \mathrm{~mm}$. Auch weitere Entzündungszeichen wie Schwellung, Rötung oder Exsudat sowie radiologische Befunde einer Periimplantitis lagen bei keinem der nachuntersuchten Patienten vor.

\subsection{Beurteilung der Patientenzufriedenheit und mundgesundheitsbezogenen Lebensqualität}

Abgesehen von den klinisch erhobenen Parametern zur Bewertung einer erfolgreichen Implantation nimmt die Meinung des Patienten und seine Zufriedenheit mit dem Behandlungsergebnis eine fast noch bedeutendere Stellung ein. Denn ein erfolgreich osseointegriertes Implantat garantiert dem Behandler noch nicht ein erfolgreiches Behandlungsergebnis und eine hohe Patientenzufriedenheit. Deshalb ist es wichtig für die abschließende Bewertung eines Behandlungskonzepts, die verschiedenen Facetten, die das Empfinden des Patienten beeinflussen, zu messen und auszuwerten. Auch Kapur et al. (1997) haben vor allem bei zahnlosen Patienten die Patientenzufriedenheit als wichtigstes Ziel einer Behandlung definiert.

In dieser Studie wurde zur Erfassung und Auswertung dieser verschiedenen Einflussfaktoren ein eigens entwickelter Fragebogen, sowie der OHIP-20 (Oral Health Impact Profile) verwendet. Der OHIP ist ein sehr differenziertes Messinstrument, das die funktionellen, physischen und auch psychischen Bereiche jedes Patienten betrachtet. Dennoch blieben für die abschließende Bewertung des Behandlungskonzepts einige zentrale Fragen offen, die durch die Hinzunahme eines weiteren Fragebogens zur Patientenzufriedenheit beantwortet werden sollten. Die entscheidenden Aspekte, die zusätzlich zum OHIP-20 beantwortet werden sollten, waren die Bewertung des Gesamtresultats der Behandlung, der Halt der Prothese, die Kaufunktion, die Anzahl der Zahnarztbesuche aufgrund von Komplikationen sowie die Weiterempfehlung der Behandlung. 
Insgesamt wurden die Fragebögen von 62 Patienten vollständig beantwortet. Das Gesamtresultat der Behandlung wurde von den meisten Patienten positiv bewertet. So benoteten $87 \%$ der befragten Patienten die Behandlung mit "sehr gut" bis "gut". Lediglich $5 \%$ vergaben die Bewertung ,ausreichend“, und nur $2 \%$ der Patienten waren unzufrieden und bewerteten mit „mangelhaft“. Dieses Ergebnis zeigt die hohe Zufriedenheit der Patienten mit dem Göttinger Behandlungskonzept der Satellitenimplantation. Der Erfolg dieses Konzepts und die hohe Patientenzufriedenheit werden zudem durch die Ergebnisse der Behandlungsempfehlung bestätigt. So würden 96,78 \% der Patienten die Behandlung einem guten Bekannten oder Freund weiterempfehlen. Auch die Bewertung der funktionellen Aspekte wurden im Mittel positiv bewertet. Die Kaufunktion wurde durchschnittlich mit der Schulnote 1,95 und die Reinigung der Prothese und Implantate mit 1,35 bewertet. Der Halt der Prothese wurde jedoch etwas schlechter mit einer durchschnittlichen Schulnote von 2,35 beurteilt. Dies wurde auch bei der Nachuntersuchung ersichtlich, da einige Patienten nach mehreren Monaten einen Retentionsverlust ihrer Prothese beklagten. Die Ursache für diesen Retentionsverlust waren auf den Verschleiß des Kugelkopfs und der Matrize zurückzuführen. In einem solchen Fall konnte durch das Aktivieren der Matrize die Retention der Prothese deutlich verbessert werden. Der Verlust der Retention und das Lockern oder Frakturieren der Matrizen stellten die häufigsten Ursachen für einen zusätzlichen Zahnarztbesuch dar. Dennoch gaben die Patienten an, dass sie im Mittel nur vier- bis fünfmal (Mittelwert 4,56) in einem mittleren Beobachtungszeitraum von 52 Monaten einen Zahnarzt aufsuchen mussten. Die Betrachtung des Median (1,5) zeigte zudem, dass bei den meisten Patienten nur ein bis zwei Zahnarztbesuche aufgrund von Komplikationen nötig waren. Bei zwei Patienten traten jedoch sehr häufig Probleme auf (50 und 37 Zahnarztbesuche), so dass der Mittelwert nicht als repräsentativ für das Gesamtkollektiv zu werten ist. Es konnten mittels Mann-Whitney-U-Test keine signifikanten Unterschiede in der Beantwortung der Fragebögen zur Patientenzufriedenheit zwischen den beiden Patientengruppen (Xive, Semados) festgestellt werden.

Der Fragebogen zur mundgesundheitsbezogenen Lebensqualität (OHIP-20) wurde insgesamt von 62 Patienten beantwortet. Bei der Auswertung der Daten wurde ein hoher mittlerer Gesamtpunktwert $(110,6)$ ermittelt, welcher nah an der maximalen Punktzahl (120) lag. Auch die Punktwerte der einzelnen Subskalen lagen relativ nah an den zu erreichenden Maximalpunktwerten. Anhand des Mann-Whitney-U-Tests konnte kein signifikanter Unterschied zwischen den beiden Implantatsystemen festgestellt werden, so dass für beide Gruppen eine vergleichbar hohe mundgesundheitsbezogene Lebensqualität angenommen werden kann. Diesen positiven Einfluss implantatgestützer Deckprothesen auf die Lebensqualität der Patienten wurde auch in weiteren Studien gezeigt (Awad und Feine (1998), Awad et al. (2003), Heydecke et al. (2005), Alfadda et al. (2009)). 
Heydecke et al. (2003) untersuchten in einer Vergleichsstudie den Einfluss implantatretinierter und konventioneller Prothesen im Unterkiefer auf die orale Lebensqualität der Patienten. Zur Erhebung der Daten wurde eine Befragung der 60 Patienten mittels OHIP-20 durchgeführt. Im Gegensatz zu unserer Studie wurde eine umgekehrte Punkteverteilung auf einer Likert-Skala von 1-6 vorgenommen. Daher repräsentierten niedrige Punktwerte (Minimalwert 20) eine höhere mundgesundheitsbezogene Lebensqualität als größere Werte (Maximalwert 120). Sechs Monate nach der Behandlung wurde für die Patienten mit einer Totalprothese ein mittlerer Gesamtpunktwert von 47,8 ermittelt. Die Patienten mit einer implantatgestützten Deckprothese erreichten hingegen einen mittleren Gesamtpunktwert von 35,0. Dieser signifikant niedrigere Punktwert lag wesentlich näher am Optimum von 20 Punkten und bestätigte das bessere Wohlbefinden der Patienten mit implantatretinierten Zahnersatz. Ein direkter Vergleich der Werte mit denen unserer Studie ist jedoch aufgrund der unterschiedlichen Skalen nicht möglich. Dennoch können in beiden Studien die Ergebnisse als Maß für eine hohe mundgesundheitsbezogene Lebensqualität interpretiert werden.

Ähnliche Ergebnisse zeigte auch eine Studie von Emami et al. (2009). Durch eine MetaAnalyse der Daten aktueller Studien sollte die Effizienz implantatretinierter Deckprothesen im Unterkiefer aus Sicht der Patienten überprüft werden. Zur Auswertung wurden alle randomisierten kontrollierten Studien in englischer und französischer Sprache bis zum Jahr 2007, die einen Vergleich konventioneller und implantatretinierter Deckprothesen durchführten, ausgewählt. Der Fokus wurde dabei vor allem auf die Patientenzufriedenheit sowie mundgesundheitsbezogene und generelle Lebensqualität der Patienten gelegt. Um die Resultate der einzelnen Studien vergleichen zu können, wurde die Effektstärke berechnet. Die zusammengefasste Effektstärke für alle Studien fiel mit einem Wert von 0,8 zugunsten der implantatretinierten Deckprothesen aus. Insgesamt wurde eine höhere Patientenzufriedenheit und orale Lebensqualität für Patienten mit implantatretinierten Deckprothesen festgestellt.

In einer Arbeit von (Woelk 2007) wurde die mundgesundheitsbezogenen Lebensqualität von Patienten mit einer Einzelzahnimplantatversorgung mit denen einer einspannigen zahngetragenen Brückenversorgung verglichen. 147 Patienten nahmen an dieser Studie teil und wurden mittels OHIP-20 befragt. Die Antworten wurden auf einer Likert-Skala (1-6) ausgewertet. Dabei stellten größere Werte eine bessere Lebensqualität dar. Die Auswertung ergab sowohl für Implantat- $(109,6)$ als auch Brückenträger $(109,2)$ hohe Summenwerte, die nah an dem Maximalwert (120) lagen. Für beide Gruppen wurden diese Ergebnisse als Hinweis auf eine hohe mundgesundheitsbezogene Lebensqualität gedeutet. Ein signifikanter Unterschied zwischen den beiden Gruppen konnte nicht festgestellt werden. Aufgrund des gleichen Messinstruments und der ähnlichen statistischen Auswertung lassen sich die Ergebnisse dieser Arbeit gut mit denen unserer Studie ver- 
gleichen. Dabei fällt auf, dass der Summenwert des OHIP-20 unserer Studie $(110,0)$ mit denen aus der Arbeit von (Woelke 2007) korreliert. Folglich kann angenommen werden, dass die Patienten unserer Studie mit einer implantatretinierten Deckprothese im Unterkiefer eine vergleichbar hohe mundgesundheitsbezogene Lebensqualität zu den Patienten mit einer festsitzenden prothetischen Versorgung aufweisen.

Für diese Studie wäre es aufschlussreich gewesen durch eine Erhebung des OHIP-20 vor der Behandlung der Patienten einen Ausgangswert zu bestimmen. Dadurch hätte der positive Effekt der Behandlung auf die orale Lebensqualität der Patienten besser veranschaulicht werden können. Zu Beginn dieser Studie war jedoch die Anwendung des OHIP-20 nicht sehr verbreitet, so dass die Hinzunahme dieses Messinstruments erst im Rahmen der Nachuntersuchungen aufkam. Des Weiteren wäre es auch interessant gewesen, die Erwartungen der Patienten an das Göttinger Konzept der Satellitenimplantation vor der Behandlung zu erfragen. In diesem Zusammenhang berichteten Allen et al. (2001) von einem starken Einfluss der eigenen Erwartungen der Patienten an die Behandlung auf deren spätere Zufriedenheit und Lebensqualität. Zusammenfassend kann jedoch auf der Grundlage beider Fragebögen eine hohe Patientenakzeptanz sowie eine erhebliche Steigerung der mundgesundheitsbezogenen Lebensqualität durch das Göttinger Behandlungskonzept der Satellitenimplantation angenommen werden.

\subsection{Schlussfolgerung}

Das Göttinger Konzept der subgingivalen kortikalen Mikrofixation liefert nach einer mittleren Beobachtungszeit von 52 Monaten positive und zuverlässige Ergebnisse. Es zeigt für die zwei verwendeten Implantatsysteme hohe Überlebensraten. Ein negativer Einfluss der Satellitenimplantate auf den periimplantären Knochenverlust sowie die periimplantäre Gingiva konnte auch nach der längeren Beobachtungszeit nicht festgestellt werden. Das Behandlungskonzept wurde von den meisten Patienten als positiv und empfehlenswert beurteilt. Durch die Behandlung wurde eine hohe Patientenzufriedenheit sowie mundgesundheitsbezogene Lebensqualität erreicht. Aufgrund der seit kurzem zunehmenden Anzahl an Publikationen, die von einer erfolgreichen Osseointegration unverblockter sofortbelasteter Implantate im interforaminalen Bereich der Mandibula berichten, ist über die Notwendigkeit einer primären Verblockung in diesem Indikationsbereich nachzudenken. Weitere Studien müssen diese Ergebnisse noch bestätigen. 


\section{Zusammenfassung}

Die Sofortbelastung dentaler Implantate ist in der Literatur bei einzelstehenden Implantaten im Unterkiefer nur wenig dokumentiert. Ziel dieser Studie war es, nach vierjähriger Beobachtungszeit den Erfolg des Konzepts der Sofortbelastung zweier interforaminaler Implantate mit einer Deckprothese im Unterkiefer unter Verwendung kortikaler Satellitenimplantate zu evaluieren.

Im Rahmen dieser prospektiven Studie wurden 86 Patienten (54 w und $32 \mathrm{~m}$, Durchschnittsalter 64 Jahre) ausgewählt, die im Zeitraum von 2001 bis 2005 mit jeweils zwei enossalen Implantaten in der Regio interforaminalis nach dem Göttinger Konzept der kortikalen Mikrofixation versorgt wurden. Ziel dieses Konzepts war es, durch die Verwendung zusätzlicher sogenannter Satellitenimplantate das eigentliche Zentralimplantat (Semados, Bego, Bremen/ Xive, Friadent, Mannheim) über einen Konnektor zu stabilisieren. Es wurden insgesamt 172 Implantate (68 Semados, 104 Xive) inseriert. Als prothetische definitive Suprakonstruktion wurde die umgearbeitete Prothese über Kugelattachments fixiert. Von den anfangs 86 operierten Patienten konnten 62 Patienten nachuntersucht und befragt werden. Die Nachuntersuchung fand im Zeitraum von 2008 - 2009 statt.

Die Überlebensrate nach Kaplan und Meier betrug in dieser Studie insgesamt 97,28 \%. Der Vergleich der Überlebensraten für die beiden Implantatsysteme ergab für das Xive-System 95,19 \% und für das Semados-System $100 \%$.

Die Analyse der Periotestwerte ergab für die Gesamtzahl der Implantate einen Mittelwert von -4,86. Beim Vergleich der beiden Implantatsysteme wurde für die Patienten mit Semados-Implantaten ein Mittelwert von -5,27 und für die Patienten mit XiveImplantaten von $-4,58$ gemessen.

Es resultierte für beide Implantatsysteme ein durchschnittlicher Knochenverlust von 1,06 mm. Bei der getrennten Auswertung der beiden Implantatsysteme wurde für das Xive-Implantat ein durchschnittlicher Knochenabbau von 1,13 mm und für das Semados-Implantat von $0,95 \mathrm{~mm}$ gemessen. Außerdem konnte durch eine Kovarianzanalyse (ANCOVA) ein signifikanter Einfluss der Verweildauer der Implantate auf den distalen Knochenabbau, sowie ein tendenzieller Einfluss auf den mesialen Knochenabbau festgestellt werden. 
Bei der Beurteilung der periimplantären Gingiva wurden für beide Implantatsysteme mittlere Sondierungstiefen von 2,46 mm ermittelt. Für die Patienten mit XiveImplantaten betrugen die Mittelwerte 2,45 mm. Für die Patienten mit Semados-Implantaten wurden mittlere Sondierungstiefentiefen von 2,47 mm gemessen. Bei beiden Implantatsystemen wurde tendenziell eine Abnahme der distalen Sondierungstiefen mit zunehmender Verweildauer der Implantate im Kiefer registriert.

Bei der Auswertung der Patientenfragebögen zur Patientenzufriedenheit bewerteten 47 \% der befragten Patienten das Gesamtresultat der Behandlung mit „sehr gut“, 40 \% mit „gut", $6 \%$ mit „befriedigend“, $5 \%$ mit ,,ausreichend“ und 2\% mit „mangelhaft". 96,78 \% der befragten Patienten würden das Göttinger Konzept der subgingivalen Satellitenimplantation weiterempfehlen. Die Bewertung der mundgesundheitsbezogenen Lebensqualität der Patienten mittels des Oral-Health-Impact-Profile zeigte einen hohen mittleren Gesamtpunktwert von 110,64 (maximale Punktzahl 120). Grundsätzlich wurde die orale Lebensqualität der Patienten als positiv eingeschätzt. 


\title{
6 Anhang
}

\subsection{Abkürzungsverzeichnis}

\author{
ANCOVA Analysis of covariance \\ DGZMK Deutsche Gesellschaft für Zahn-, Mund- und Kieferheilkunde \\ EMBASE Excerpta Medica Database \\ IG Implantatgruppe \\ ISQ Implant Stability Quotient \\ ITI International Team for Implantology \\ Max. $\quad$ Maximum \\ Min. Minimum \\ mm Milimeter \\ MW Mittelwert \\ N Newton \\ NIH National Institutes of Health \\ OHIP Oral Health Impact Profile \\ OHRQL Oral health-related quality of life \\ OPAN Orthopantomogramm \\ PTW Periotestwert \\ RFA Resonanz-Frequenz-Analyse \\ SF-36 Short Form Health Survey- 36 \\ Std.abw. Standardabweichung \\ TPG Totalprothesengruppe
}




\subsection{Tabellenverzeichnis}

1.1 Literaturübersicht der Frühbelastung von $2009-2010 \ldots \ldots \ldots \ldots \ldots$

1.2 Literaturübersicht der Frühbelastung von $2007-2009$. . . . . . . . . 6

1.3 Literaturübersicht der Sofortbelastung von 2007-2009 . . . . . . . . 10

1.4 Literaturübersicht der Sofortbelastung von 2006-2007 . . . . . . . . . 11

2.2 Internationale verwendete Erfolgskriterien für enossale dentale Implantate 24

2.4 Die Antwortoptionen des OHIP-20-Fragebogens (Woelk 2007) . . . . . 29

2.6 OHIP-Subskaleneinteilung (Woelk 2007) . . . . . . . . . . . . . 30

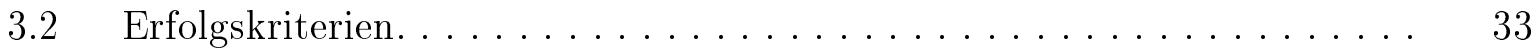

3.3 Übersicht der Periotestwerte . . . . . . . . . . . . . . . . 34

3.4 Ergebnisse der Kovarianzanalyse für die Periotestwerte . . . . . . . . . . 37

3.5 Messdaten des horizontalen Knochenabbaus . . . . . . . . . . . . . 37

3.6 Ergebnisse der Kovarianzanalyse für den horizontalen Knochenabbau . . 40

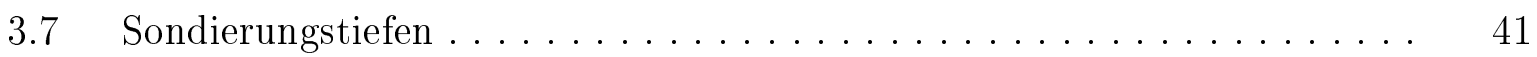

3.8 Ergebnisse der Kovarianzanalyse für die Sondierungstiefen. . . . . . . . . 43

3.9 Ergebnisse der Kovarianzanalyse für Sondierungsblutungen . . . . . . . . . 44

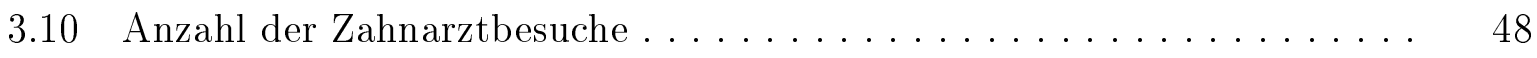

3.11 Ergebnisse des OHIP - Fragebogens. . . . . . . . . . . . . . 50

\subsection{Abbildungsverzeichnis}

$1.1 \quad$ Schematische Darstellung der Satellitenimplantate . . . . . . . . . . . 19

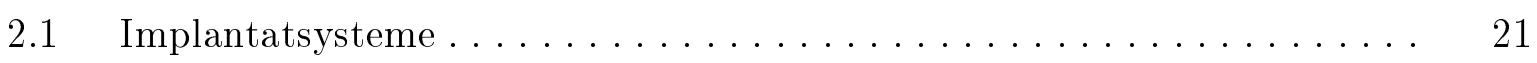

2.2 Satellitenimplantat und Konnektor . . . . . . . . . . . . . . 22

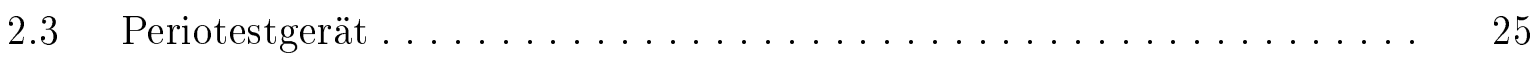

2.4 Digitalisierte OPAN-Aufnahme in Sidexis-Programm importiert . . . . . 26

2.5 Längenmessung des Implantates vor Kalibrierung. . . . . . . . . . . . 27

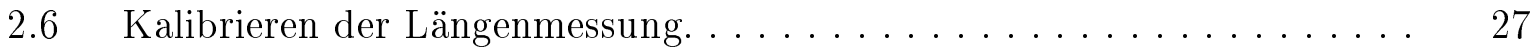

$2.7 \quad$ Ausmessen des horizontalen Knochenabbaus. . . . . . . . . . . . . . 28

$3.1 \quad$ Altersverteilung der Patientengruppen . . . . . . . . . . . 31

3.2 Kaplan-Meier-Kurve $($ gesamt $) \ldots \ldots \ldots \ldots \ldots \ldots \ldots \ldots \ldots \ldots \ldots \ldots \ldots$

$3.3 \quad$ Kaplan-Meier-Kurve $($ Semados, Xive) . . . . . . . . . . . . . . 33 
3.4 Verteilung der Periotestwerte (in Prozent) $\ldots \ldots \ldots \ldots \ldots \ldots \ldots \ldots$

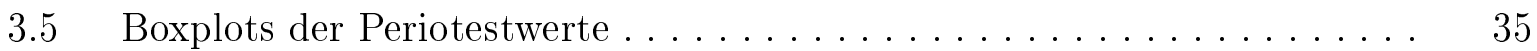

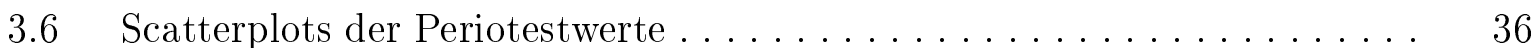

$3.7 \quad$ Knochenabbau (in $\mathrm{mm}):$ Implantattyp . . . . . . . . . . . . 38

$3.8 \quad$ Knochenabbau (in $\mathrm{mm}$ ) : Implantatposition. . . . . . . . . . . . . . . 39

3.9 horizontaler Knochenabbau (distal) : Zeit in situ (Monate) . . . . . . . 39

3.10 horizontaler Knochenabbau (mesial) : Zeit in situ (Monate)........ 40

3.11 Mittelwert/Fehlerplot für Sondierungstiefen : Implantattyp. . . . . . . . 41

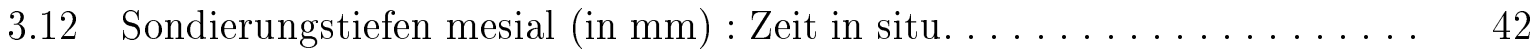

3.13 Sondierungstiefen distal (in mm) : Zeit in situ . . . . . . . . . . . . . . 42

3.14 Anzahl der Blutungen . . . . . . . . . . . . . . . . . . 43

3.15 Patientenbewertung des Gesamtresultats . . . . . . . . . . . . . . 44

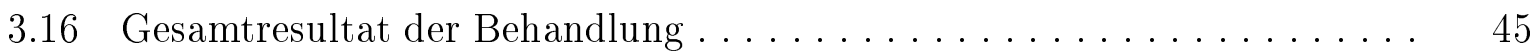

3.17 Patientenbewertung des Halts der Prothese . . . . . . . . . . . 45

3.18 Halt der Prothese $\ldots \ldots \ldots \ldots \ldots \ldots \ldots \ldots \ldots \ldots \ldots$

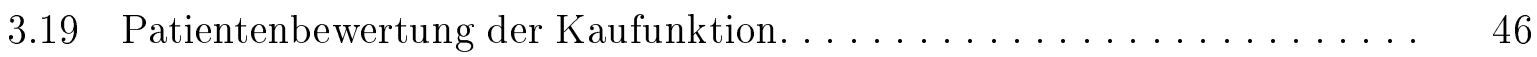

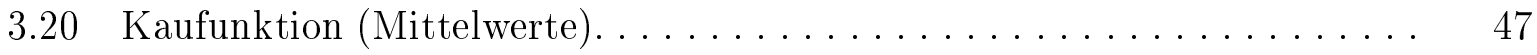

3.21 Patientenbewertung der Reinigung der Prothese/Implantate . . . . . . . . 47

3.22 Reinigung der Prothese . . . . . . . . . . . . . . . . . . . . 48

3.23 Mittelwerte der OHIP-Subskalen (Semados/Xive) . . . . . . . . . . . 50

3.24 Orthopantomogramm mit quadrangulärem Referenzkörper Regio 033

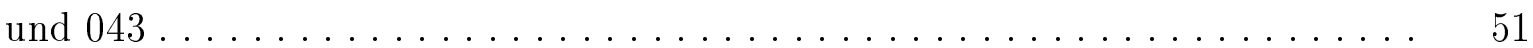

3.25 Orthopantomogramm nach Implantation mit Satellitenimplantaten . . . . 52

3.26 Umgearbeitete UK-Prothese mit Matrizen . . . . . . . . . . . . . . 52

3.27 Orthopantomogramm sechs Jahre nach Implantation . . . . . . . . . 53

3.28 Intraorale Aufnahme 6 Jahre nach Implantation . . . . . . . . . . . 54

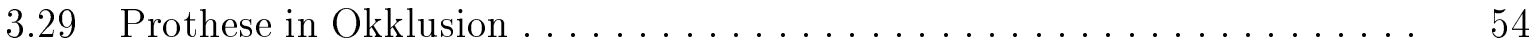




\section{Literaturverzeichnis}

Adell R, Lekholm U, Rockler B, Brånemark PI (1981): A 15-year study of osseointegrated implants in the treatment of the edentulous jaw. Int J Oral Surg 10, 387-416

Albrektsson T (2001): Is surgical skill more important for clinical success than changes in implant hardware? Clin Implant Dent Relat Res $\underline{3}, 174-175$

Albrektsson T, Zarb G, Worthington P, Eriksson AR (1986): The long-term efficacy of currently used dental implants: a review and proposed criteria of success Int J Oral Maxillofac Implants $\underline{1}, 11-25$

Alfadda SA, Attard NJ and David LA (2009): Five-year clinical results of immediately loaded dental implants using mandibular overdentures. Int J Prosthodont $\underline{22}$ 368-373

Allen PF, Locker D (1997): Do item weights matter? An assessment using the oral health impact profile. Community Dent Health $\underline{14}, 133-138$

Allen PF and Locker D (2002): A modified short version of the oral health impact profile for assessing health-related quality of life in edentulous adults. Int J Prosthodont $\underline{15}$, $446-450$

Allen PF, McMillan AS and Walshaw D (2001): A patient-based assessment of implantstabilized and conventional complete dentures. J Prosthet Dent $\underline{85}, 141-147$

Allison P, Locker D, Jokovic A, Slade G (1999): A cross-cultural study of oral health values. J Dent Res $\underline{78}, 643-649$

Alsabeeha N, Atieh M, Payne AGT (2009): Loading Protocols for Mandibular Implant Overdentures: A Systematic Review with Meta-Analysis. Clin Implant Dent Relat Res (im Druck)

Aparicio C (1997): The use of the Periotest value as the initial success criteria of an implant: 8-year report. Int J Periodontics Restorative Dent 17, 150-161

Apse P, Ellen RP, Overall CM, Zarb GA (1989): Microbiota and crevicular fluid collagenase activity in the osseointegrated dental implant sulcus: a comparison of sites in edentulous and partially edentulous patients. J Periodontal Res $\underline{24}$, 96-105 
Arvidson K, Esselin O, Felle-Persson E, Jonsson G, Smedberg JI, Soderstrom U (2008): Early loading of mandibular full-arch bridges screw retained after 1 week to four to five Monotype implants: 3-year results from a prospective multicentre study. Clin Oral Implants Res $\underline{19}$, 693-703

Assad AS, Hassan SA, Shawky YM, Badawy MM (2007): Clinical and radiographic evaluation of implant-retained mandibular overdentures with immediate loading. Implant Dent $\underline{16}, 212-223$

Assunção WG, Zardo GG, Delben JA, Barão VAR (2007): Comparing the efficacy of mandibular implant-retained overdentures and conventional dentures among elderly edentulous patients: satisfaction and quality of life. Gerodontology 24 , 235-238

Assunção WG, Barão VAR, Delben JA, Gomes EA, Tabata LF (2009): A comparison of patient satisfaction between treatment with conventional complete dentures and overdentures in the elderly: a literature review. Gerodontology 27, 154-162

Astrand P, Engquist B, Dahlgren S, Gröndahl K, Engquist E, Feldmann H (2004): Astra Tech and Brånemark system implants: a 5-year prospective study of marginal bone reactions. Clin Oral Implants Res $\underline{15}$, 413-420

Awad MA, Feine JS (1998): Measuring patient satisfaction with mandibular prostheses. Community Dent Oral Epidemiol 26, 400-405

Awad MA, Lund JP, Shapiro SH, Locker D, Klemetti E, Chehade A, Savard A, Feine JS (2003): Oral health status and treatment satisfaction with mandibular implant overdentures and conventional dentures: a randomized clinical trial in a senior population. Int J Prosthodont 16, 390-396

BDIZ (2006): 1. Europäische Konsensuskonferenz Implantologie (European Consensus Conference, EuCC)

Berglundh T, Lindhe j, Jonsson K, Ericsson I (1994): The topography of the vascular systems in the periodontal and peri-implant tissues in the dog. J Clin Periodontol $\underline{3}$, $189-193$

Block MS, Kent JN, Finger IM (1990): Use of the integral implant for overdenture stabilization. Int J Oral Maxillofac Implants $\underline{5}, 140-147$

Boerrigter EM, Stegenga B, Raghoebar GM, Boering G (1995): Patient satisfaction and chewing ability with implant-retained mandibular overdentures: a comparison with new complete dentures with or without preprosthetic surgery. J Oral Maxillofac Surg $\underline{53}$, $1167-1173$ 
Brånemark PI (1983): Osseointegration and its experimental background. J Prosthet Dent $\underline{50}, 399-410$

Brånemark PI, Engstrand P, Ohrnell LO, Gröndahl K, Nilsson P, Hagberg K, Darle C, Lekholm U (1999): Brånemark Novum: a new treatment concept for rehabilitation of the edentulous mandible. Preliminary results from a prospective clinical follow-up study. Clin Implant Dent Relat Res $1,2-16$

Bruyn HD, Besseler J, Raes F, Vaneker M (2009): Clinical outcome of overdenture treatment on two nonsubmerged and nonsplinted Astra Tech Microthread implants. Clin Implant Dent Relat Res $\underline{11}, 81-89$

Bruyn HD, Collaert B (2002): Early loading of machined-surface Brånemark implants in completely edentulous mandibles: healed bone versus fresh extraction sites. Clin Implant Dent Relat Res $\underline{4}, 136-142$

Buch RSR, Weibrich G, Wagner W (2003): Criteria of success in implantology. Mund Kiefer Gesichtschir 7, 42-46

Buser D, Weber HP, Lang NP (1990): Tissue integration of non-submerged implants. 1-year results of a prospective study with 100 ITI hollow-cylinder and hollow-screw implants. Clin Oral Implants Res $\underline{1}, 33-40$

Buser D, Nydegger T, Hirt HP, Cochran DL, Nolte LP (1998): Removal torque values of titanium implants in the maxilla of miniature pigs. Int J Oral Maxillofac Implants $\underline{13}, 611-619$

Cehreli MC, Uysal S, Akca K (2009): Marginal Bone Level Changes and Prosthetic Maintenance of Mandibular Overdentures Supported by 2 Implants: A 5-Year Randomized Clinical Trial. Clin Implant Dent Relat Res 12, 114-121

Chiapasco M, Gatti C (2003): Implant-retained mandibular overdentures with immediate loading: a 3- to 8-year prospective study on 328 implants. Clin Implant Dent Relat Res $\underline{5}, 29-38$

Chiapasco M, Abati S, Romeo E, Vogel G (2001): Implant-retained mandibular overdentures with Brånemark System MKII implants: a prospective comparative study between delayed and immediate loading. Int J Oral Maxillofac Implants $\underline{16}, 537-546$

Cooper LF, Moriarty JD, Guckes AD, Klee LB, Smith RG, Almgren C, Felton DA (2008): Five-year prospective evaluation of mandibular overdentures retained by two microthreaded, TiOblast nonsplinted implants and retentive ball anchors. Int J Oral Maxillofac Implants $\underline{23}, 696-704$ 
Degidi M, Piattelli A, Iezzi G, Carinci F (2007): Retrospective study of 200 immediately loaded implants retaining 50 mandibular overdentures. Quintessence Int $\underline{38}, 281-288$

DGZMK (2000): Statement der DGZMK zur Lebenserwartung von Implantaten und Implantatlager. DZZ 55

d'Hoedt B, Lukas D, Mühlbradt L, Scholz F, Schulte W, Quante F, Topkaya A (1985): [Periotest methods-development and clinical trial]. Dtsch Zahnarztl Z $\underline{40}, 113-125$

Ellis JS, Burawi G, Walls A, Thomason JM (2009): Patient satisfaction with two designs of implant supported removable overdentures; ball attachment and magnets. Clin Oral Implants Res_20, 1293-1298

Emami E, Heydecke G, Rompré PH, de Grandmont P, Feine JS (2009): Impact of implant support for mandibular dentures on satisfaction, oral and general health-related quality of life: a meta-analysis of randomized-controlled trials. Clin Oral Implants Res $\underline{20}, 533-544$

Engelke W, Diederichs CG, Jacobs HG, Deckwer I (1997): Alveolar reconstruction with splitting osteotomy and microfixation of implants. Int J Oral Maxillofac Implants $\underline{12}$, $310-318$

Engelke W, Stahr S, Schwarzwäller W (2002): Erhöhung der Primärstabilität dentaler Implantate durch Satellitenimplantate. Eine in Vitro-Untersuchung. Oralchirurgie Journal $\underline{2}, 12-15$

Engelke W, Decco OA, de las Mercedes Capobianco M, Schwarzwäller W, Villavicencio MM (2005): Immediate occlusal loading of freestanding implants using cortical satellite implants: preliminary report of a prospective study. Implant Dent $\underline{14}, 50-57$

Friberg B, Henningsson C, Jemt T (2005): Rehabilitation of edentulous mandibles by means of turned Brånemark System implants after one-stage surgery: a 1-year retrospective study of 152 patients. Clin Implant Dent Relat Res $\underline{7}, 1-9$

Gallucci GO, Morton D, Weber H-P (2009): Loading protocols for dental implants in edentulous patients. Int J Oral Maxillofac Implants 24, 132-146

Glauser R, Lundgren AK, Gottlow J, Sennerby L, Portmann M, Ruhstaller P, Hämmerle CHF (2003): Immediate occlusal loading of Brånemark TiUnite implants placed predominantly in soft bone: 1-year results of a prospective clinical study. Clin Implant Dent Relat Res $\underline{5}, 47-56$ 
Gómez-Román G, Axmann D, d'Hoedt B, Schulte W (1995): Eine Methode zur qua ntitativen Erfassung und statistischen Auswertung des periimplantären Knochenabbaues. Stomatologie $\underline{92}, 463-471$

Gualini F, Gualini G, Cominelli R, Lekholm U (2009): Outcome of Brånemark Novum implant treatment in edentulous mandibles: a retrospective 5-year follow-up study. Clin Implant Dent Relat Res $\underline{11}, 330-337$

Hays RD, Ware JE (1986): My medical care is better than yours. Social desirability and patient satisfaction ratings. Med Care $\underline{24}, 519-524$

Heckmann SM, Winter W, Meyer M, Weber HP, Wichmann MG (2001): Overdenture attachment selection and the loading of implant and denture-bearing area. Part 2: A methodical study using five types of attachment. Clin Oral Implants Res 12, 640-647

Henry PJ, van Steenberghe D, Blombäck U, Polizzi G, Rosenberg R, Urgell JP, Wendelhag I (2003): Prospective multicenter study on immediate rehabilitation of edentulous lower jaws according to the Brånemark Novum protocol. Clin Implant Dent Relat Res $\underline{5}, 137-142$

Heydecke G, Locker D, Awad MA, Lund JP, Feine JS (2003): Oral and general healthrelated quality of life with conventional and implant dentures. Community Dent Oral Epidemiol 31, 161-168

Heydecke G, Thomason JM, Lund JP, Feine JS (2005): The impact of conventional and implant supported prostheses on social and sexual activities in edentulous adults Results from a randomized trial 2 months after treatment. J Dent 33, 649-657

Hultin M, Fischer J, Gustafsson A, Kallus T, Klinge B (2000): Factors affecting late fixture loss and marginal bone loss around teeth and dental implants. Clin Implant Dent Relat Res 2, 203-208

ITI-Konsensuskonferenz (2004): Proceedings of the Third ITI (International Team for Implantology) Consensus Conference. Gstaad, Switzerland, August 2003. Int J Oral Maxillofac Implants $\underline{19}, 7-154$

Jahn M, d'Hoedt B (1992): Zur Definition des Erfolges bei dentalen Implantaten. Z Zahnärztl Imp $\underline{8}, 221-226$

Jennings KJ (1991): ITI hollow-cylinder and hollow-screw implants: prosthodontic management of edentulous patients using overdentures. Int J Oral Maxillofac Implants $\underline{6}$, 202-206 
Kaplan EL, Meier P (1958): Nonparametric estimation from incomplete observations. J Am Stat Assoc 53, 457-462

Kapur KK, Garrett NR, Dent RJ, Hasse AL (1997): A randomized clinical trial of two basic removable partial denture designs. Part II: Comparisons of masticatory scores. J Prosthet Dent $\underline{78}, 15-21$

Kawai Y, Taylor JA (2007): Effect of loading time on the success of complete mandibular titanium implant retained overdentures: a systematic review. Clin Oral Implants Res $\underline{18}, 399-408$

Kieswetter K, Schwartz Z, Dean DD, Boyan BD (1996): The role of implant surface characteristics in the healing of bone. Crit Rev Oral Biol Med_z, 329-345

King GN, Hermann JS, Schoolfield JD, Buser D, Cochran DL (2002): Influence of the size of the microgap on crestal bone levels in non-submerged dental implants: a radiographic study in the canine mandible. J Periodontol $\underline{73}, 1111-1117$

Koberg W (1985): Unwanted late results following augmentative alveolar ridge-plasty of the mandible using autologous rib transplants. Fortschr Kiefer Gesichtschir 30, 41-46

Lambrecht JT, Filippi A, Künzel AR and Schiel HJ (2003): Long-term evaluation of submerged and nonsubmerged ITI solid-screw titanium implants: a 10-year life table analysis of 468 implants. Int J Oral Maxillofac Implants $\underline{18}, 826-834$

Lekholm U, Zarb G: Patient selection and preparation. in: Tissue integrated prostheses; osseointegration in clinical dentistry; hrsg. v. Branemark P, Zarb G, Albrektsson T; Quintessence International, Berlin, Chicago, London, San Paulo, Tokio 1985, 69-123.

Ledermann PD (1977): Complete denture provision of atrophic problem mandible with aid of CBS-implants. Quintessenz $\underline{28}, 21-26$

Ledermann PD (1979): Bar-prosthetic management of the edentulous mandible by means of plasma-coated implantation with titanium screws. Dtsch Zahnärztl Z 34 , 907-911

Ledermann PD, Schenk RK, Buser D (1998): Long-lasting osseointegration of immediately loaded, bar-connected TPS screws after 12 years of function: a histologic case report of a 95-year-old patient. Int J Periodontics Restorative Dent $\underline{18}, 552-563$

Liao K-Y, Kan JY, Rungcharassaeng K, Lozada JL, Herford AS, Goodacre CJ (2010): Immediate loading of two freestanding implants retaining a mandibular overdenture: 1-year pilot prospective study. Int J Oral Maxillofac Implants 25, 784-790 
Liddelow GJ, Henry PJ (2007): A prospective study of immediately loaded single implant-retained mandibular overdentures: preliminary one-year results. J Prosthet Dent $\underline{97}, 126-137$

Ma S, Payne AGT (2010): Marginal bone loss with mandibular two-implant overdentures using different loading protocols: a systematic literature review. Int J Prosthodont_23, $117-126$

Marzola R, Scotti R, Fazi G and Schincaglia GP (2007): Immediate loading of two implants supporting a ball attachment-retained mandibular overdenture: a prospective clinical study. Clin Implant Dent Relat Res $\underline{9}$, 136-143

Meredith N, Friberg B, Sennerby L, Aparicio C (1998): Relationship between contact time measurements and PTV values when using the Periotest to measure implant stability. Int J Prosthodont 11, 269-275

Mericske-Stern R (1997): Force distribution on implants supporting overdentures: the effect of distal bar extensions. A 3-D in vivo study. Clin Oral Implants Res $\underline{8}$, 142-151

Meyer U, Joos U, Mythili J, Stamm T, Hohoff A, Fillies T, Stratmann U, Wiesmann HP (2004): Ultrastructural characterization of the implant/bone interface of immediately loaded dental implants. Biomaterials 25, 1959-1967

Naert I, Quirynen M, van Steenberghe D, Darius P (1992): A six-year prosthodontic study of 509 consecutively inserted implants for the treatment of partial edentulism. J Prosthet Dent $\underline{67}, 236-245$

Noguerol B, Muñoz R, Mesa F, de Dios Luna J, O’Valle F (2006): Early implant failure. Prognostic capacity of Periotest: retrospective study of a large sample. Clin Oral Implants Res 17, 459-464

Olivé J, Aparicio C (1990): Periotest method as a measure of osseointegrated oral implant stability. Int J Oral Maxillofac Implants $\underline{5}$, 390-400

Ormianer Z, Garg AK, Palti A (2006): Immediate loading of implant overdentures using modified loading protocol. Implant Dent 15, 35-40

Payne AGT, Tawse-Smith A, Duncan WD, Kumara R (2002): Conventional and early loading of unsplinted ITI implants supporting mandibular overdentures. Clin Oral Implants Res $\underline{13}$, 603-609

Quirynen M, Naert I, van Steenberghe D, Teerlinck J, Dekeyser C, Theuniers G (1991): Periodontal aspects of osseointegrated fixtures supporting an overdenture. A 4-year retrospective study. J Clin Periodontol_18, 719-728 
Raghoebar GM, Friberg B, Grunert I, Hobkirk JA, Tepper G, Wendelhag I (2003): 3-year prospective multicenter study on one-stage implant surgery and early loading in the edentulous mandible. Clin Implant Dent Relat Res $\underline{5}, 39-46$

Reinhardt S, Drüke B (1993): Vier verschiedene Versorgungskonzepte des zahnlosen Unterkiefers. Dtsch Zahnärztl Z $\underline{48}, 805$

Romeo E, Chiapasco M, Lazza A, Casentini P, Ghisolfi M, Iorio M, Vogel G (2002): Implant-retained mandibular overdentures with ITI implants. Clin Oral Implants Res $\underline{13}, 495-501$

Rungcharassaeng K, Kan JY (2000): Immediately loaded mandibular implant bar overdenture: a surgical and prosthodontic rationale. Int J Periodontics Restorative Dent 20, $71-79$

Rutkunas V, Mizutani H, Puriene A (2008): Conventional and early loading of twoimplant supported mandibular overdentures. A systematic review. Stomatologija 10, $51-61$

Schenk RK, Buser D (1998): Osseointegration: a reality. Periodontol 17, 22-35

Schnitman PA, Shulman LB (1979): Recommendations of the consensus development conference on dental implants. J Am Dent Assoc 98, 373-377

Schnitman PA, Shulman LB (1980): Dental implants. Benefit and risk.Proceedings of an NIH Harvard Consensus Development Conference 1978. National Institute of Health (NIH) Harvard Consensus Development Conference. NIH, US Department of Health and Human Services Bethesda, 81-1531

Schou S, Holmstrup P, Stoltze K, Hjørting-Hansen E, Fiehn N-E, Skovgaard LT (2002): Probing around implants and teeth with healthy or inflamed peri-implant mucosa/gingiva. A histologic comparison in cynomolgus monkeys (Macaca fascicularis). Clin Oral Implants Res $\underline{13}, 113-126$

Siadat H, Alikhasi M, Mirfazaelian A, Geramipanah F, Zaery F (2008): Patient satisfaction with implant-retained mandibular overdentures: a retrospective study. Clin Implant Dent Relat Res 10, 93-98

Slade GD (1997): Derivation and validation of a short-form oral health impact profile. Community Dent Oral Epidemiol 25, 284-290

Slade GD (1998): Assessing change in quality of life using the Oral Health Impact Profile. Community Dent Oral Epidemiol 26, 52-61 
Slade GD, Spencer AJ (1994): Development and evaluation of the Oral Health Impact Profile. Community Dent Health $\underline{11}, 3-11$

Smet ED, Duyck J, Sloten JV, Jacobs R, Naert I (2007): Timing of loading-immediate, early, or delayed-in the outcome of implants in the edentulous mandible: a prospective clinical trial. Int J Oral Maxillofac Implants 22, 580-594

Snauwaert K, Duyck J, van Steenberghe D, Quirynen M, Naert I (2000): Time dependent failure rate and marginal bone loss of implant supported prostheses: a 15-year follow-up study. Clin Oral Investig 4 , 13-20

Spiekermann H, Jansen VK, Richter EJ (1995): A 10-year follow-up study of IMZ and TPS implants in the edentulous mandible using bar-retained overdentures. Int J Oral Maxillofac Implants $\underline{10}, 231-243$

Stoker GT, Wismeijer D (2008): Prosthetic dilemmas. Immediate versus conventional loading of implants in the edentulous mandible. Ned Tijdschr Tandheelkd 115, 589-595

Stoker GT, Wismeijer D (2009): Immediate Loading of Two Implants with a Mandibular Implant-Retained Overdenture: A New Treatment Protocol. Clin Implant Dent Relat Res (im Druck)

Tawse-Smith A, Payne AGT, Kumara R, Thomson WM (2002): Early loading of unsplinted implants supporting mandibular overdentures using a one-stage operative procedure with two different implant systems: a 2-year report. Clin Implant Dent Relat Res $\underline{4}, 33-42$

Tetsch P, Ackermann K, Behneke N, Galandi M, Geis-Gerstorfer J, Kerschbaum T, Krämer A, Krekeler G, Nentwig G, Richter E (1990): Konsensus-Konferenz zur Implantologie, 18.10.1989 in Mainz. Z Zahnärztl Implantol_[5, 5-14

Turkyilmaz I, Tözüm TF, Tumer C, Ozbek EN (2006a): A 2-year clinical report of patients treated with two loading protocols for mandibular overdentures: early versus conventional loading. J Periodontol 77, 1998-2004

Turkyilmaz I, Tumer C, Avci M, Hersek N, Celik-Bagci E (2006b): A short-term clinical trial on selected outcomes for immediately loaded implant-supported mandibular overdentures. Int J Prosthodont 19, 515-519

Turkyilmaz I, Suarez JC, Company AM, McGlumphy EA (2009a): Early load mandibular hybrid prosthesis using the Ohio State University acrylic frame requiring no final impression. Aust Dent J $\underline{54}, 255-261$ 
Turkyilmaz I, Tözüm TF, Tumer C (2009b): Early versus Delayed Loading of Mandibular Implant-Supported Overdentures: 5-Year Results. Clin Implant Dent Relat Res 12, $39-46$

Vercruyssen M, Marcelis K, Coucke W, Naert I, Quirynen M (2010): Long-term, retrospective evaluation (implant and patient-centred outcome) of the two-implantssupported overdenture in the mandible. Part 1: survival rate. Clin Oral Implants Res 21, 357-365

Woelk G: Lebensqualität und Zufriedenheit mit implantatgetragenem und konventionellen Zahnersatz. Med. Diss. Freiburg 2007 


\section{Danksagung}

Ganz besonders danke ich

meinem Doktorvater, Herrn Prof. Dr. Dr. Wilfried Engelke für die Überlassung des Themas, die hervorragende Betreuung sowie die Ansprechbarkeit und Hilfsbereitschaft bei Fragen und Problemen,

Frau Katharina Lange für die statistischen Berechnungen der Messergebnisse sowie die umfassende Betreuung. 
